TRANSACTIONS OF THE

AMERICAN MATHEMATICAL SOCIETY

Volume 362, Number 8, August 2010, Pages 4385-4431

S 0002-9947(10)05034-8

Article electronically published on March 12, 2010

\title{
COUNTABLE GROUPS OF ISOMETRIES ON BANACH SPACES
}

\author{
VALENTIN FERENCZI AND ELÓI MEDINA GALEGO
}

\begin{abstract}
A group $G$ is representable in a Banach space $X$ if $G$ is isomorphic to the group of isometries on $X$ in some equivalent norm. We prove that a countable group $G$ is representable in a separable real Banach space $X$ in several general cases, including when $G \simeq\{-1,1\} \times H, H$ finite and $\operatorname{dim} X \geq$ $|H|$, or when $G$ contains a normal subgroup with two elements and $X$ is of the form $c_{0}(Y)$ or $\ell_{p}(Y), 1 \leq p<+\infty$. This is a consequence of a result inspired by methods of S. Bellenot (1986) and stating that under rather general conditions on a separable real Banach space $X$ and a countable bounded group $G$ of isomorphisms on $X$ containing $-I d$, there exists an equivalent norm on $X$ for which $G$ is equal to the group of isometries on $X$.

We also extend methods of K. Jarosz (1988) to prove that any complex Banach space of dimension at least 2 may be renormed with an equivalent complex norm to admit only trivial real isometries, and that any complexification of a Banach space may be renormed with an equivalent complex norm to admit only trivial and conjugation real isometries. It follows that every real Banach space of dimension at least 4 and with a complex structure may be renormed to admit exactly two complex structures up to isometry, and that every real Cartesian square may be renormed to admit a unique complex structure up to isometry.
\end{abstract}

\section{INTRODUCTION}

What groups $G$ may be seen as the group of isometries on a Banach space $X$ ? This general question may be formulated by the following definition given by K. Jarosz in 1988.

Definition 1 (Jarosz [11). A group $G$ is representable in a Banach space $X$ if there exists an equivalent norm on $X$ for which the group of isometries on $X$ is isomorphic to $G$.

In [11, Jarosz stated as an open question which groups were representable in a given Banach space. The difference with the classical theory of representation of groups on linear spaces is that here we require an isomorphism with the group of isometries on a Banach space, and not just some group of isometries or isomorphisms. Since $\{-I d, I d\}$ is always a normal subgroup of the group of isometries on a real Banach space, it follows that a group which is representable in a real Banach space must always contain a normal subgroup with two elements. Conversely,

Received by the editors June 13, 2007 and, in revised form, March 2, 2009.

2000 Mathematics Subject Classification. Primary 46B03, 46B04.

Key words and phrases. Group of isometries on Banach spaces, group representable in a Banach space, complex structures up to isometry. 
J. Stern [19] proved that for any group $G$ which contains a normal subgroup with two elements, there exists a real Hilbert space $H$ such that $G$ is representable in $H$. Furthermore if $G$ is countable, then $H$ may be chosen to be separable.

For an arbitrary Banach space $X$ it remains open which groups are representable in $X$. Jarosz proved that $\{-1,1\}$ is representable in any real Banach space, and that the unit circle $\mathbb{C}_{1}$ is representable in any complex space (the separable real case had been solved previously by S. Bellenot [2]). He also proved that for any countable group $G,\{-1,1\} \times G$ is representable in $C([0,1])$, and that for any group $G$ there exists a complex space $X$ such that $\mathbb{C}_{1} \times G$ is representable in $X$.

In a first section of this paper, we give a much more general answer to the question of representability by proving that:

- The group $\{-1,1\} \times G$ is representable in $X$ whenever $G$ is a finite group and $X$ a separable real space $X$ such that $\operatorname{dim} X \geq|G|$ (Theorem 17).

- The group $G$ is representable in $X$ whenever $G$ is a countable group admitting a normal subgroup with two elements and $X$ is a separable real Banach space with a symmetric decomposition either isomorphic to $c_{0}(Y)$ or to $\ell_{p}(Y)$ for some $Y$ and $1 \leq p<+\infty$, or with the Radon-Nikodym Property (Theorem 19).

- The group $\{-1,1\} \times G$ is representable in $X$ whenever $G$ is a countable group and $X$ an infinite-dimensional separable real Banach space containing a complemented subspace with a symmetric basis (Theorem 21).

These results are partial answers to a conjecture of Jarosz who asked whether $\{-1,1\} \times G$ is representable in $X$ for any group $G$ and any real space $X$ such that $\operatorname{dim} X \geq|G|$.

As an application of our results we obtain that a countable group $G$ is representable in $c_{0}$ (resp. $C([0,1]), \ell_{p}$ for $1 \leq p<+\infty, L_{p}$ for $\left.1 \leq p<+\infty\right)$ if and only if it contains a normal subgroup with two elements (Corollary 20).

Our method is to ask, given a group $G$ of linear isomorphisms on a real Banach space $X$, whether there exists an equivalent norm on $X$ for which $G$ is the group of isometries on $X$. Once the problem of representability is reduced to representing a given group as some group of isomorphisms on a given Banach space, it is much simpler to address, and this leads to Theorem 17. Theorem 19, and Theorem 21. In other words, we explore in which respect the question of representability of groups in Banach spaces belongs to the renorming theory or rather may be reduced to the purely isomorphic theory.

If a group of isomorphisms is the group of isometries on a real (resp. complex) Banach space in some equivalent norm, then it must be bounded, contain $-I d$ (resp. $\lambda I d$ for all $\lambda \in \mathbb{C}_{1}$ ), and be closed for the topology of the strong convergence of $T$ and $T^{-1}$. Therefore the question is:

Question 2. Let $X$ be a real (resp. complex) Banach space and let $G$ be a group of isomorphisms on $X$ which is bounded, contains $-I d$ (resp. $\lambda I d$ for all $\lambda \in \mathbb{C}_{1}$ ), and is closed for the topology of the strong convergence of $T$ and $T^{-1}$. Does there exist an equivalent norm on $X$ for which $G$ is the group of isometries on $X$ ?

A positive answer was obtained by $\mathrm{Y}$. Gordon and R. Loewy 9 when $X=\mathbb{R}^{n}$ and $G$ is finite (in which case of course the boundedness and closedness hypotheses are automatically satisfied). This answered a question by J. Lindenstrauss. In this 
paper, we extend the methods of Bellenot and use a result of V. Kadec, see [4] p. 48, or a renorming method of G. Lancien [14] to considerably improve this result:

- Let $X$ be a separable real Banach space. Then for any finite group $G$ of isomorphisms on $X$ which contains $-I d$, there exists an equivalent norm on $X$ for which $G$ is equal to the group of isometries on $X$ (Theorem 11).

- Let $X$ be a separable real Banach space with the Radon-Nikodym Property. Then for any countable bounded group $G$ of isomorphisms on $X$ which contains $-I d$ and is separated by some point with discrete orbit, there exists an equivalent norm on $X$ for which $G$ is equal to the group of isometries on $X$ (Theorem 12).

Therefore for separable real spaces and finite groups, the question of representability really does not belong to renorming theory. Also, note that a countable group of isomorphisms on $X$ which is equal to the group of isometries in some equivalent norm must always be discrete for the topology of the strong convergence of $T$ and $T^{-1}$ and admit a separating point (Lemma 13). It remains unknown however whether this implies the existence of a separating point with discrete orbit, that is, if the implication in Theorem 12 is an equivalence for countable groups.

To conclude that section we deduce Theorem 17. Theorem 19] and Theorem 21 essentially from Theorem 11 and Theorem 12, We also prove that Theorem 17 and Theorem 21 are optimal in the sense that there exist a real space in which representable finite groups are exactly those of the form $\{-1,1\} \times G$ and a real space containing a complemented subspace with a symmetric basis in which representable countable groups are exactly those of the form $\{-1,1\} \times G$; see Proposition 22 and Proposition 23 On the other hand we have the classical examples of $c_{0}$, $C([0,1]), \ell_{p}, 1 \leq p<+\infty$ and $L_{p}, 1 \leq p<+\infty$ for which Corollary 20 states that representable countable groups are exactly those which admit a normal subgroup with two elements, and we also provide an intermediary example of a space in which the class of representable finite groups is strictly contained in between the above two classes; see Proposition 24

In a second section of this paper, we use the renorming methods of Jarosz in [1] to study complex structures on real Banach spaces up to isometry. Our results are actually related to the representability of the circle group $\mathbb{C}_{1}$ and of the group of isometries on $\mathbb{C}$ as the group of $\mathbb{R}$-linear isometries on a complex Banach space.

We recall a few facts about complex structures. An introduction to this subject may also be found in [5. Any complex Banach space is also a real Banach space, and conversely, the linear structure on a real Banach space $X$ may be induced by a $\mathbb{C}$-linear structure; the corresponding complex Banach space is said to be a complex structure on $X$ in the isometric sense. It is clear that any complex structure on $X$ is canonically associated to some $\mathbb{R}$-linear map $I$ on $X$ such that $I^{2}=-I d$ and $\cos \theta I d+\sin \theta I$ is an isometry for all $\theta$, and which defines the multiplication by the imaginary number $i$. Conversely for any such map $I$, there exists an associated complex structure denoted $X^{I}$, defined for $a, b \in \mathbb{R}$ and $x \in X$ by

$$
(a+i b) \cdot x=a x+b I x .
$$

The existing theory of complex structures, however, concerns existence and uniqueness of a complex structure up to isomorphism. In this case, complex structures correspond to real isomorphisms $I$ of square $-I d$, and conversely such operators define a complex structure by the same procedure as above and under the 
equivalent norm $\||\cdot \||$ defined by $\|\left|x\left\|\mid=\max _{\theta}\right\| \cos \theta x+\sin \theta I x \|\right.$. It is well known that complex structures do not always exist up to isomorphism on a Banach space. By [3], 12] there exist real spaces with at least two complex structures up to isomorphism, and the examples of [3] and [1] (which are separable) actually admit a continuum of complex structures. By [6] for each $n \in \mathbb{N}^{*}$ there exists a space with exactly $n$ complex structures up to isomorphism. In [6] and [7] various examples of spaces different from the classical example of $\ell_{2}$ are also shown to have a unique complex structure up to isomorphism, including an $\mathrm{HI}$ example, a space with an unconditional basis, and a $C(K)$ space defined by Plebanek.

It actually turns out that the classical spaces $c_{0}, C([0,1]), \ell_{p}, 1 \leq p \leq+\infty$ and $L_{p}, 1 \leq p<+\infty$ also admit a unique complex structure up to isomorphism. A nice and simple proof of this fact was given to us by N.J. Kalton after a first version of our paper was written and is included here; see Theorem 28.

The isometric theory of complex structures turns out to be totally different from the isomorphic theory. For a very general class of Banach spaces $X$, we show that quite various situations may be obtained concerning existence and uniqueness of complex structures up to isometry on $X$ by choosing different renormings on $X$. This may justify why the isometric theory of complex structures has not been investigated before, as it is unclear what other results one may want to obtain in that area.

We first prove that $\ell_{2}$ has a unique complex structure up to isometry; see Proposition 26. On the other hand, since Jarosz [11] showed that every real Banach space may be renormed to admit only trivial isometries (i.e. the only isometries are $I d$ and $-I d)$, every real Banach space may be renormed not to admit complex structures in the isometric sense.

Extending the methods of Jarosz [11] we prove that:

- Any complex Banach space of dimension at least 2 may be renormed to admit only trivial real isometries (Corollary 45).

- Any complex Banach space which is real isomorphic to a Cartesian square, and whose complex law is the canonical one associated to the decomposition as a square, may be renormed to admit only trivial and conjugation isometries (Corollary 46).

It follows, Theorem 30 .

- Every real Banach space of dimension at least 4 and with a complex structure may be renormed to admit exactly two complex structures up to isometry.

- Every real Cartesian square may be renormed to admit, as a unique complex structure up to isometry, the canonical complex structure associated to its decomposition as a square.

In a last section we extend results of F. Rabiger and W.J. Ricker, [17, about isometries on complex spaces of the type of Gowers and Maurey, by proving that any isometry on a real Banach space on which any operator is a strictly singular perturbation of a multiple of the identity must be of the form $\pm I d+K, K$ compact; see Proposition 49. This applies to Gowers and Maurey's hereditarily indecomposable space $G M,[10$.

For classical results in Banach space theory, such as, for example, results concerning the Radon-Nikodym Property or symmetric bases, we shall refer to [15]; for renorming questions in Banach spaces, we shall refer to [4]. 


\section{REPRESENTATION OF COUNTABLE GROUPS ON SEPARABLE REAL BANACH SPACES}

2.1. G-pimple norms on separable Banach spaces. In [2], Bellenot showed how to renorm a given separable real Banach space so that the group of isometries in the new norm is equal to $\{-I d, I d\}$. In this subsection we extend the construction of Bellenot to an arbitrary countable group of isometries containing $-I d$ instead of $\{-I d, I d\}$. So in what follows, $X$ is real separable, $G$ is a countable group of isometries on $X$ containing $-I d$, and under certain conditions on $G$, we construct an equivalent norm on $X$ for which $G$ is the group of isometries on $X$.

In [2], Bellenot renorms $X$ with an LUR norm and then defines, for some $x_{0}$ in $X$ of norm 1, a new unit ball (which he calls the "pimple" ball) obtained by adding two small cones in $x_{0}$ and $-x_{0}$. Any isometry in the new norm must preserve the cones and therefore send $x_{0}$ to $\pm x_{0}$. Repeating this for a sequence $\left(x_{n}\right)_{n}$ with dense linear span, chosen carefully so that one can add the cones "independently", and so that the sizes of the cones are "sufficiently" different, any isometry sends $x_{n}$ to $\pm x_{n}$. Finally, if each $x_{n}$ was chosen "much closer" to $x_{0}$ than to $-x_{0}$, any isometry fixing $x_{0}$ must fix each $x_{n}$ and therefore any isometry is equal to $I d$ or $-I d$.

In our case, fixing some $x_{0}$ in $X$ of norm 1 , one should obviously put cones of the same size in each $g x_{0}, g \in G$, defining a "G-pimple ball", so that any isometry in the new norm preserves the orbit $G x_{0}$. This first step could be realized even when $G$ is not countable. Then one repeats a similar procedure as in [2, adding other cones in $g x_{n}, g \in G$ for a sufficiently dense sequence $\left(x_{n}\right)_{n}$, so that any isometry preserves $G x_{n}$ for all $n$. These $x_{n}$ 's for $n \geq 1$ are said to be of type 1 . Finally, a last step is added to only allow as isometries isomorphisms whose restriction to $G x_{0}$ is a permutation which corresponds to the action of some $g \in G$ on $G x_{0}$. This is technically more complicated and is obtained by adding cones at some points of span $G x_{0}$ which code the structure of $G$ and are said to be of type 2 . The fact that $G$ is countable and $X$ separable guarantees that there are at most countably many cones, which will allow us to provide lower estimates for the distances between two such cones.

The reader may get a geometric feeling of this proof by looking at the group $G=\{ \pm I d, \pm R\}$ of $\mathbb{R}$-linear isometries on $\mathbb{C}$, where $R$ is the rotation of angle $\pi / 2$. By adding cones "of type 1 " on the unit ball at \pm 1 and $\pm i$, one allows the isometries in $G$ but also symmetries with respect to the axes. A way of correcting this is to add one well-placed smaller cone "of type 2 " next to each element of $\{ \pm 1, \pm i\}$, for example in $\pm e^{i \pi / 6}$ and $\pm e^{i 2 \pi / 3}$, so that the only isometries in the new norm are those of $G$.

Definition 3. Let $X$ be a real Banach space with norm $\|$.$\| , let G$ be a group of isometries on $X$ such that $-I d \in G$, and let $\left(x_{k}\right)_{k \in K}$ be a possibly finite sequence of normalized vectors of $X$. Let $\Lambda=\left(\lambda_{k}\right)_{k \in K}$ be such that $1 / 2<\lambda_{k}<1$ for all $k \in K$. The $(\Lambda, G)$-pimple at $\left(x_{k}\right)_{k}$ for $\|$.$\| is the equivalent norm on X$ defined by

$$
\|y\|_{\Lambda, G}=\inf \left\{\sum\left[\left[y_{i}\right]\right]_{\Lambda, G}: y=\sum y_{i}\right\}
$$

where $[[y]]_{\Lambda, G}=\lambda_{k}\|y\|$, whenever $y \in \operatorname{span}\left(g \cdot x_{k}\right)$ for some $k \in K$ and $g \in G$, and $[[y]]_{\Lambda, G}=\|y\|$ otherwise. 
In other words, the closed unit ball for $\|\cdot\|_{\Lambda, G}$ is the convexification of the union of the closed unit ball for $\|$.$\| with line segments between g x_{k} / \lambda_{k}$ and $-g x_{k} / \lambda_{k}$ for each $k \in K$ and $g \in G$.

In [2], Bellenot had defined the notion of a $\lambda$-pimple at $x_{0} \in X$, which corresponds to $((\lambda),\{-I d, I d\})$-pimple in our terminology: that is, the closed unit ball of the $\lambda$-pimple at $x_{0}$ is the convexification of the closed unit ball for $\|$.$\| and of a$ single line segment between $x_{0} / \lambda$ and $-x_{0} / \lambda$.

It may be observed that

$$
\left(\inf _{k \in K} \lambda_{k}\right)\|\cdot\| \leq\|\cdot\|_{\Lambda, G} \leq\|\cdot\|,
$$

so that $\|\cdot\|_{\Lambda, G}$ is an equivalent norm on $X$, and that by definition, any $g \in G$ remains an isometry in the norm $\|\cdot\|_{\Lambda, G}$.

Recall that a norm $\|$.$\| is locally uniformly rotund (or LUR) at some point x$, $\|x\|=1$, if for all $\epsilon \in] 0,2]$, there exists $\lambda(x, \epsilon)<1$ such that whenever $\|y\|=1$ and $\|x-y\| \geq \epsilon$, it follows that $\left\|\frac{x+y}{2}\right\| \leq \lambda(x, \epsilon)$. Equivalently $\lim _{n} x_{n}=x$ whenever $\lim _{n}\left\|x_{n}\right\|=\|x\|$ and $\lim _{n}\left\|x+x_{n}\right\|=2\|x\|$. It is LUR when it is LUR at all points of the unit sphere. As in 2 we shall say that a normalized vector $y$ is extremal for a norm $\|$.$\| if it is an extremal point of the closed unit ball for \|$.$\| , that is, if$ whenever $\|y\|=\|z\|=1$ and $x$ belongs to the segment $[y, z]$, then $y=x=z$. A norm is strictly convex at a point $x$ of the unit sphere if $x$ is extremal. A norm is strictly convex when it is strictly convex at all points of the unit sphere. Note that if a norm is LUR at $x$, then it must be strictly convex at $x$. More details about these notions may be found in 4 .

We recall a crucial result from [2] (Proposition, p. 90).

Proposition 4 (Bellenot [2]). Let $(X,\|\|$.$) be a real Banach space and let x_{0} \in X$ be normalized such that

(1) $\|$.$\| is LUR at x_{0}$, and

(2) there exists $\epsilon>0$ such that if $\|y\|=1$ and $\left\|x_{0}-y\right\|<\epsilon$, then $y$ is an extremal point for $\|$.$\| .$

Then given $\delta>0, b>0$ and $0<m<1$, there exists a real number $1 / 2<\lambda_{0}<1$ such that whenever $\lambda_{0} \leq \lambda<1$ and $\|.\|_{\lambda}$ is the $\lambda$-pimple at $x_{0}$, then

(3) $m\|\cdot\| \leq\|\cdot\|_{\lambda} \leq\|\cdot\|$,

(4) if $1=\|y\|>\|y\|_{\lambda}$, then $\left\|x_{0}-y\right\|<\delta$ or $\left\|x_{0}+y\right\|<\delta$,

(5) $x_{\lambda}=\lambda^{-1} x_{0}$ is the only isolated extremal point for $\|.\|_{\lambda}$ which satisfies $\|x /\| x\left\|-x_{0}\right\|<\epsilon$,

(6) if $w$ is a vector so that $x_{\lambda}$ and $x_{\lambda}+w$ are endpoints of a maximal line segment in the unit sphere of $\|\cdot\|_{\lambda}$, then $b \geq\|w\| \geq \lambda^{-1}-1$.

Our objective is to generalize this result to $(\Lambda, G)$-pimples in a natural manner which for the $\Lambda$ part is directly inspired from [2].

In the following, $\|\cdot\|_{\Lambda, G}$ is as before the $(\Lambda, G)$-pimple associated to a given sequence $\left(x_{k}\right)_{k}$. We let $B$ denote the closed unit ball for $\|\cdot\|, B_{\Lambda}^{G}$ denote the closed unit ball for the $(\Lambda, G)$-pimple at $\left(x_{k}\right)_{k}, B_{k}^{g}$ denote the closed unit ball for the $\lambda_{k}$-pimples $\|\cdot\|_{\lambda_{k}, g}$ at $g x_{k}$, and $B_{0}$ denote the union over $k \in K$ and $g \in G$ of the closed unit balls $B_{k}^{g}$. It is clear that $B \subset B_{0} \subset B_{\Lambda}^{G}$.

If $\Lambda=\left(\lambda_{k}\right)_{k \in K}, \Lambda^{\prime}=\left(\lambda_{k}^{\prime}\right)_{k \in K}$, and $c, d \in \mathbb{R}$, we write $\Lambda<\Lambda^{\prime}$ to mean $\lambda_{k}<\lambda_{k}^{\prime}$ for all $k \in K, c<\Lambda$ to mean $c<\lambda_{k}$ for all $k \in K$, and $\Lambda<d$ to mean $\lambda_{k}<d$ for all $k \in K$. 
We shall first prove a lemma, Lemma 5. stating that for $\Lambda$ close enough to $1, B_{\Lambda}^{G}$ is actually equal to $B_{0}$. This will allow us to prove Proposition [6, our generalization of Bellenot's results, by reducing essential parts of the argument to the simple application of Proposition 4 in each $g x_{k}$, that is, to the case of a single $\lambda_{k}$-pimple associated to the single point $g x_{k}$.

Lemma 5. Let $(X,\|\|$.$) be a real Banach space, let G$ be a group of isometries on $X$ containing $-I d$ and let $\left(x_{k}\right)_{k \in K}$ be a finite or infinite sequence of normalized vectors of $X$. Assume

(1) $\|$.$\| is strictly convex on X$, and LUR at $x_{k}$ for each $k \in K$, and

(2) for all $k \in K, c_{k}:=\inf \left\{\left\|x_{j}-g x_{k}\right\|: j \in K, g \in G,(j, g) \neq(k, I d)\right\}>0$.

Then there exists $\Lambda_{0}=\left(\lambda_{0 k}\right)_{k}$, with $1 / 2<\Lambda_{0}$, such that whenever $\Lambda=\left(\lambda_{k}\right)_{k}$ satisfies $\Lambda_{0}<\Lambda<1$, then

(a) whenever $x \in B_{k}^{g} \backslash B$ and $y \in B_{l}^{h} \backslash B$ with $(k, g) \neq(l, \pm h)$, then $\|x-y\| \geq$ $c_{\min (k, l)} / 3$.

(b) The closed unit ball $B_{\Lambda}^{G}$ of the $(\Lambda, G)$-pimple at $\left(x_{k}\right)_{k}$ is equal to the union $B_{0}$ over $k \in K$ and $g \in G$ of the closed unit balls $B_{k}^{g}$ of the $\lambda_{k}$-pimples $\|\cdot\|_{\lambda_{k}, g}$ at $g x_{k}$.

(c) The following equality holds: $\|\cdot\|_{\Lambda, G}=\inf _{k \in K, g \in G}\|\cdot\|_{\lambda_{k}, g}$.

(d) Whenever $\|x\|_{\Lambda, G}<\|x\|$, there exists a unique pair $\{-g, g\} \subset G$ and a unique $k \in K$ such that $\|x\|_{\lambda_{k}, g}<\|x\|$, and in this case $\|x\|_{\Lambda, G}=\|x\|_{\lambda_{k}, g}$.

Furthermore for each $k, \lambda_{0 k}$ depends only on $x_{i}, c_{i}, 1 \leq i \leq k$.

Proof. Note that by (1), \|.\| is LUR at $x_{k}$ for each $k$, so Proposition廿(1) is satisfied for $x_{0}=x_{k}$. By (1) again, $\|$.$\| is strictly convex, and therefore any normalized vector$ $y$ is extremal for $\|$.$\| , so Proposition 4(2)$ applies in $\left(x_{k}\right)$ for any choice of $\epsilon>0$. We let $\epsilon_{k}=c_{k} / 2$, and fix a decreasing sequence $\left(\delta_{k}\right)_{k}$ such that for all $k \geq 1, \delta_{k} \leq c_{k} / 4$ and $\frac{4 \delta_{k}}{3} \leq 1-\lambda\left(x_{k}, c_{k}\right)$, where $\lambda\left(x_{k},.\right)$ is the LUR function associated to the norm $\|$.$\| in x_{k}$.

For each $k$, we let $\lambda_{0 k}$ be the real $\lambda_{0}$ associated by Proposition 4 to $x_{0}=x_{k}$, $\epsilon=\epsilon_{k}, \delta=\delta_{k}, b=1$ and $m=1 / 2$. Up to replacing each $\lambda_{0 k}$ by a larger number in ]1/2, 1 [, we may assume that $\lambda_{0 k}^{-1}-1 \leq \delta_{k} / 3$ for all $k \in K$ and that $\lim _{k \rightarrow+\infty} \lambda_{0 k}=1$ if $K$ is infinite.

We let $\Lambda=\left(\lambda_{k}\right)_{k}$ be such that $\Lambda_{0}<\Lambda<1$.

We first prove (a). Whenever $x \in B_{k}^{g} \backslash B$ we have, by Proposition 4 (4), that $\left\|z-g x_{k}\right\|<\delta_{k}$ or $\left\|z+g x_{k}\right\|<\delta_{k}$, where $z=x /\|x\|$. Up to redefining $g$ as $-g$ if necessary we may assume that the first holds. Then

$$
\left\|x-g x_{k}\right\| \leq\|z-x\|+\left\|z-g x_{k}\right\|<\|x\|-1+\delta_{k} \leq \lambda_{k}^{-1}-1+\delta_{k} \leq \frac{4 \delta_{k}}{3} .
$$

Likewise if $y \in B_{l}^{h} \backslash B$, then up to redefining $h$ as $-h$,

$$
\left\|y-h x_{l}\right\|<\frac{4 \delta_{l}}{3} .
$$

If now $x \in B_{k}^{g} \backslash B$ and $y \in B_{l}^{h} \backslash B$ with $(k, g) \neq(l, \pm h)$ and $k \leq l$, we have that

$$
\|x-y\| \geq\left\|g x_{k}-h x_{l}\right\|-\left\|x-g x_{k}\right\|-\left\|y-h x_{l}\right\| \geq c_{k}-\frac{4}{3 \delta_{k}}-\frac{4}{3 \delta_{l}},
$$


so since $\delta_{l} \leq \delta_{k}$,

$$
\|x-y\| \geq c_{k}-\frac{8 \delta_{k}}{3} \geq c_{k} / 3
$$

Therefore (a) is proved.

We now intend to prove (b). First we observe that $B_{0}$ is closed. Indeed let $x$ be the limit of a convergent sequence $\left(x_{n}\right)$ in $B_{0}$, and let $k_{n}, g_{n}$ be such that $x_{n} \in B_{k_{n}}^{g_{n}}$ : we claim that $x \in B_{0}$. Indeed if $x_{n} \in B$ for infinitely many $n$, then $x \in B$ and we are done, so we may assume that $x_{n} \in B_{k_{n}}^{g_{n}} \backslash B$ for each $n$. If $k_{n}$ is bounded, then we may assume that $k_{n}$ is constantly equal to some $k$ and that $\left\|x_{m}-x_{n}\right\|<c_{k} / 3$ for all $n, m$. But then by (a), $B_{k}^{g_{n}}=B_{k}^{g_{m}}$ for all $n, m$, so $x$ also belongs to $B_{k}^{g_{n}}$ for any choice of $n$ and therefore to $B_{0}$, and we are done. So we may assume that $k_{n}$ converges to $+\infty$; then since $\lambda_{0 k_{n}}$ converges to $1, \lambda_{k_{n}}$ also converges to 1 , and since $\left\|x_{n}\right\| \leq 1 / \lambda_{k_{n}}$ for each $n,\|x\| \leq 1$. Therefore $x \in B \subset B_{0}$, which proves the claim. Finally $B_{0}$ is closed.

Next we observe that $B_{0}$ is convex. Assuming towards a contradiction that $x, y \in B_{0}$ and $\frac{x+y}{2} \notin B_{0}$, let $(k, g)$ and $(l, h)$ be such that $x \in B_{k}^{g}$ and $y \in B_{l}^{h}$, and without loss of generality assume that $k \leq l$. By convexity of $B_{k}^{g}$ and $B_{l}^{h}$, these two balls are different; otherwise $\frac{x+y}{2}$ would belong to either of them and therefore to $B_{0}$. Furthermore $x \in B_{k}^{g} \backslash B$; otherwise $x \in B \subset B_{l}^{h}$ and $\frac{x+y}{2} \in B_{l}^{h} \subset B_{0}$. In other words, $\|x\|_{\lambda_{k}, g}<\|x\|$. Likewise $\|y\|_{\lambda_{l}, h}<\|y\|$. Therefore by Proposition 4 (4) applied to $x /\|x\|$ for the $\lambda_{k}$-pimple at $g x_{k}$, and up to replacing $g$ by $-g$ if necessary, $\left\|g x_{k}-x /\right\| x\|\|<\delta_{k}$. Then

$$
\left\|g x_{k}-x\right\| \leq\left\|g x_{k}-\frac{x}{\|x\|}\right\|+\left\|x-\frac{x}{\|x\|}\right\| \leq \delta_{k}+\lambda_{k}^{-1}-1 \leq \frac{4 \delta_{k}}{3} .
$$

Likewise $\left\|h x_{l}-y\right\|<\frac{4 \delta_{l}}{3}$. Then

$$
\left\|\frac{x+y}{2}-\frac{g x_{k}+h x_{l}}{2}\right\| \leq \frac{2\left(\delta_{k}+\delta_{l}\right)}{3} \leq \frac{4 \delta_{k}}{3} .
$$

Since $\left\|g x_{k}-h x_{l}\right\| \geq c_{k}$ by (2), it follows by LUR of $\|\cdot\|$ in $g x_{k}$ that

$$
\left\|\frac{g x_{k}+h x_{l}}{2}\right\| \leq \lambda\left(g x_{k}, c_{k}\right)=\lambda\left(x_{k}, c_{k}\right) \text {, }
$$

and

$$
\left\|\frac{x+y}{2}\right\| \leq \frac{4 \delta_{k}}{3}+\lambda\left(x_{k}, c_{k}\right) \leq 1,
$$

a contradiction, since $\frac{x+y}{2}$ does not belong to $B_{0}$ and therefore neither to $B$. This contradiction proves that $B_{0}$ is convex.

Finally we have proved that $B_{0}$ is closed convex. Since it contains $B$ and each segment $\left[-g x_{k} / \lambda_{k}, g x_{k} / \lambda_{k}\right]$, it therefore contains $B_{\Lambda}^{G}$, and also since $B_{0}$ is included in $B_{\Lambda}^{G}$, (b) is proved.

The equality in (c),

$$
\|\cdot\|_{\Lambda, G}=\inf _{k \in K, g \in G}\|\cdot\|_{\lambda_{k}, g}
$$

follows immediately from (b).

To prove (d), let $x$ be such that $\|x\|_{\Lambda, G}<\|x\|$. Then by (c) there exist $\lambda_{k}, g$ such that $\|x\|_{\lambda_{k}, g}<\|x\|$. Therefore $z=x /\|x\|_{\lambda_{k}, g} \in B_{k}^{g} \backslash B$. Since in (a), the real $c_{\min (k, l)}$ is positive, there exists no other $B_{l}^{h}$ such that $z \in B_{l}^{h} \backslash B$. In other words, $z \notin B_{l}^{h}$ for $(k, g) \neq(l, \pm h)$. 
If we had that $\|x\|_{\lambda_{l}, h}<\|x\|$ for some $(l, h) \neq(k, \pm g)$, then $z^{\prime}=x /\|x\|_{\lambda_{l}, h}$ would belong to $B_{l}^{h} \backslash B$. If $\|x\|_{\lambda_{l}, h} \leq\|x\|_{\lambda_{k}, g}$, then by convexity of $B_{\lambda_{l}}^{h}, z \in B_{\lambda_{l}}^{h}$ and so $z \in B_{\lambda_{l}}^{h} \backslash B$, a contradiction. If $\|x\|_{\lambda_{l}, h} \geq\|x\|_{\lambda_{k}, g}$, then we obtain a similar contradiction using $z^{\prime}$. Therefore $\|x\|_{\lambda_{l}, h} \geq\|x\|$. Finally we have proved that $k$ and $\{-g, g\}$ are unique so that $\|x\|_{\lambda_{k}, g}<\|x\|$. From (c) we therefore deduce that $\|x\|_{\Lambda, G}=\|x\|_{\lambda_{k}, g}$. This concludes the proof of (d).

Summing up, we see that if $x$ is a point such that $\|x\|_{\Lambda, G}<\|x\|$, then $\|x\|_{\Lambda, G}=$ $\|x\|_{\lambda_{k}, g}$ for some $\left(\lambda_{k}, g\right)$ such that $\|x\|_{\lambda_{k}, g}<\|x\|,\left(\lambda_{k}, g\right)$ and $\left(\lambda_{k},-g\right)$ are the only pairs to share this property, and for all other $\left(\lambda_{l}, h\right),\|x\|_{\lambda_{l}, h}=\|x\|$.

Proposition 6. Let $(X,\|\|$.$) be a real Banach space, let G$ be a group of isometries on $X$ containing $-I d$ and let $\left(x_{k}\right)_{k \in K}$ be a finite or infinite sequence of normalized vectors of $X$. Assume

(1) $\|$.$\| is strictly convex on X$ and LUR at $x_{k}$ for each $k \in K$, and

(2) for all $k \in K, c_{k}:=\inf \left\{\left\|x_{j}-g x_{k}\right\|: j \in K, g \in G,(j, g) \neq(k, I d)\right\}>0$.

Then given $\left(\delta_{k}\right)_{k}>0,\left(b_{k}\right)_{k}>0$ and $0<m<1$, there exists $\Lambda_{0}=\left(\lambda_{0 k}\right)_{k}$, with $1 / 2<\Lambda_{0}$ such that whenever $\Lambda=\left(\lambda_{k}\right)_{k}$ satisfies $\Lambda_{0}<\Lambda<1$, then

$\left(3^{\prime}\right) m\|\cdot\| \leq\|\cdot\|_{\Lambda, G} \leq\|\cdot\|$,

$\left(4^{\prime}\right)$ if $1=\|y\|>\|y\|_{\Lambda, G}$, then $\exists g \in G, k \in K:\left\|g x_{k}-y\right\|<\delta_{k}$,

(5') $x_{k, \lambda}=\lambda_{k}^{-1} x_{k}$ is the only isolated extremal point of $\|\cdot\|_{\Lambda, G}$ which satisfies $\|x /\| x\left\|-x_{k}\right\|<c_{k} / 2$,

$\left(6^{\prime}\right)$ if $w$ is a vector so that $x_{k, \lambda}$ and $x_{k, \lambda}+w$ are endpoints of a maximal line segment in the unit sphere of $\|\cdot\|_{\Lambda, G}$, then $b_{k} \geq\|w\| \geq \lambda_{k}^{-1}-1$.

Furthermore for each $k, \lambda_{0 k}$ depends only on $m$ and $x_{i}, c_{i}, \delta_{i}, b_{i}, 1 \leq i \leq k$.

Proof. Fix $G,\left(x_{k}\right)_{k \in K}$ and $\left(\delta_{k}\right)_{k}>0,\left(b_{k}\right)_{k}>0$ and $0<m<1$ as in the hypotheses. We may again assume that $\left(\delta_{k}\right)_{k}$ is decreasing and that for all $k \geq 1, \delta_{k} \leq c_{k} / 4$ and $\frac{3 \delta_{k}}{2} \leq 1-\lambda\left(x_{k}, c_{k}\right)$, and we let $\epsilon_{k}=c_{k} / 2$.

Note that as in Lemma 5. by (1), Proposition 4 (1) is satisfied for $x_{0}=x_{k}$ and Proposition 4 (2) applies in $\left(x_{k}\right)$ for any choice of $\epsilon>0$.

Therefore, for each $k$, let $\lambda_{0 k}$ be the $\lambda_{0}$ given by Proposition 4 for $x_{0}=x_{k}$, $\epsilon=\epsilon_{k}, \delta=\delta_{k}, b=b_{k}$ and $m$. Up to replacing each $\lambda_{0 k}$ by a larger number in ]1/2,1[, we may also assume that (a) to (d) of Lemma 5 are satisfied whenever $\Lambda=\left(\lambda_{k}\right)_{k}$ is such that $\Lambda_{0}<\Lambda<1$.

We now fix some $\Lambda$ such that $\Lambda_{0}<\Lambda<1$ and verify $\left(3^{\prime}\right)$ to $\left(6^{\prime}\right)$.

Affirmation $\left(3^{\prime}\right)$ is obvious from Proposition 4 (3) for each $\left(\lambda_{k}, g\right)$, that is,

$$
m\|\cdot\| \leq\|\cdot\|_{\lambda_{k}, g} \leq\|\cdot\|,
$$

and from Lemma 5 (c), that is,

$$
\|\cdot\|_{\Lambda, G}=\inf _{k \in K, g \in G}\|\cdot\|_{\lambda_{k}, g} .
$$

For $\left(4^{\prime}\right)$ assume $1=\|y\|>\|y\|_{\Lambda, G}$. Then by Lemma $\mathbf{5}$ (d), there exist $g, k$ such that $1=\|y\|>\|y\|_{\lambda_{k}, g}$, so from Proposition 4 (4) applied for $\|\cdot\|_{\lambda_{k}, g},\left\|g x_{k}-y\right\|<$ $\delta_{k}$ or $\left\|-g x_{k}-y\right\|<\delta_{k}$. This proves $\left(4^{\prime}\right)$.

To prove $\left(5^{\prime}\right)$ we note that if $\|x /\| x\left\|-x_{k}\right\|<c_{k} / 2$, then whenever $g \neq \pm I d$ or $k \neq l$,

$$
\|x /\| x\left\|-g x_{l}\right\|>\left\|g x_{l}-x_{k}\right\|-c_{k} / 2 \geq c_{k} / 2 \geq \delta_{k},
$$


and likewise

$$
\|x /\| x\left\|+g x_{l}\right\| \geq \delta_{k} .
$$

Applying Proposition 4 (4) to $y=x /\|x\|$ and $\|\cdot\|_{\lambda_{l}, h}$ we deduce that

$$
\|x\|=\|x\|_{\lambda_{l}, h}
$$

whenever $(l, h) \neq(k, \pm g)$. From Lemma 5 (c) it follows that

$$
\|x\|_{\Lambda, G}=\|x\|_{\lambda_{k}, g} .
$$

Therefore for all those $x$ such that $\|x /\| x\left\|-x_{k}\right\|<\epsilon_{k}, x$ is an isolated extremal point for $\|\cdot\|_{\Lambda, G}$ if and only if it is an isolated extremal point for $\|\cdot\|_{\lambda_{k}, g}$, which, by an application of Proposition 4 (5) for $x_{k}$ and $\epsilon_{k}=c_{k} / 2$, is equivalent to saying that $x=x_{k, \Lambda}$. Therefore $\left(5^{\prime}\right)$ is proved.

The proof of $\left(6^{\prime}\right)$ is a little bit longer. We denote by $S_{k}^{g}$ the unit sphere for $\|\cdot\|_{\lambda_{k}, g}$, by $S_{\Lambda}^{G}$ the unit sphere for $\|\cdot\|_{\Lambda, G}$, by $S$ the unit sphere for $\|\cdot\|$, and by $S^{\prime}$ the set of points of $S$ on which $\|\cdot\|_{\Lambda, G}=\|\cdot\|$. By Lemma 5 (c)(d), $S_{\Lambda}^{G}=S^{\prime} \cup\left(\bigcup_{k, g}\left(S_{k}^{g} \backslash S\right)\right)$.

We prove the following result:

Claim. A line segment in $S_{\Lambda}^{G}$ cannot contain points both in $S_{k}^{g} \backslash S$ and $S_{l}^{h} \backslash S$ with $S_{k}^{g} \neq S_{l}^{h}$.

Proof. First note that by Lemma 5 (a), whenever $x \in S_{k}^{g} \backslash S, y \in S_{l}^{h} \backslash S$, with $S_{k}^{g} \neq S_{l}^{h}$, it follows that $d(x, y) \geq c_{\min (k, l)} / 3$. Therefore whenever $y \in S_{l}^{h} \backslash S$, we have that

$$
d\left(y, S_{k}^{g} \backslash S\right) \geq d_{k} / 3,
$$

where $d_{k}:=\min _{l \leq k} c_{l}>0$. Assume a line segment $L$ in $S_{\Lambda, G}$ contained a point $x$ in $S_{k}^{g} \backslash S$ and a point $y$ in $S_{l}^{h} \backslash S$ with $S_{l}^{h} \neq S_{k}^{g}$. By the above, $d\left(y, S_{k}^{g} \backslash S\right) \geq d_{k} / 3$. Since also $d\left(x, S_{k}^{g} \backslash S\right)=0$, we could define $x^{\prime} \neq y^{\prime}$ in the line segment $[x, y]$ such that $d\left(z, S_{k}^{g} \backslash S\right) \in\left[d_{k} / 9,2 d_{k} / 9\right]$ whenever $z \in\left[x^{\prime}, y^{\prime}\right]$. For any such $z$ we would have that

$$
z \notin S_{k^{\prime}}^{g^{\prime}} \backslash S,
$$

whenever $S_{k^{\prime}}^{g^{\prime}} \neq S_{k}^{g}$, because $d\left(z, S_{k}^{g} \backslash S\right) \leq 2 d_{k} / 9<\inf _{w \in S_{k^{\prime}}^{g^{\prime}} \backslash S} d\left(w, S_{k}^{g} \backslash S\right)$, and also that

$$
z \notin S_{k}^{g} \backslash S
$$

because $d\left(z, S_{k}^{g} \backslash S\right) \geq d_{k} / 9>0$. Since $z$ belongs to $S_{\Lambda}^{G}$ and $S_{\Lambda}^{G}=S^{\prime} \cup$ $\left(\bigcup_{k, g}\left(S_{k}^{g} \backslash S\right)\right)$, this would mean that $z$ belongs to $S^{\prime}$ and therefore to $S$. Then the nontrivial line segment $\left[x^{\prime}, y^{\prime}\right]$ would be included in $S$, but this would contradict the strict convexity of $\|$.$\| . This concludes the proof of the claim.$

Going back to $x_{k, \Lambda}$, since this vector belongs to $S_{k}^{I d} \backslash S$, we deduce from the claim and from the formula $S_{\Lambda}^{G}=S^{\prime} \cup\left(\bigcup_{k, g}\left(S_{k}^{g} \backslash S\right)\right)$ that if $\left[x_{k, \Lambda}, x_{k, \Lambda}+w\right]$ is a maximal line segment in $S_{\Lambda}^{G}$, it is a line segment in $\left(S_{k}^{I d} \backslash S\right) \cup S^{\prime} \subset S_{k}^{I d}$. We shall now prove that this segment cannot be extended in $S_{k}^{I d}$.

To show this, note that for any strict extension $\left[x_{k, \lambda}, y\right]$ of $\left[x_{k, \Lambda}, x_{k, \Lambda}+w\right]$ in $S_{k}^{I d}$, either $\left[x_{k, \lambda}, y\right] \subset S_{k}^{I d} \backslash S \subset S_{\Lambda}^{G}$ by Lemma 5 (c)(d), and in this case the maximality in $S_{\Lambda}^{G}$ is contradicted, or there exists a sequence $\left(y_{n}\right)_{n}$ of distinct points converging to $x_{k, \Lambda}+w$ in the segment $\left[x_{k, \lambda}, y\right]$ with $y_{n} \in S$ for all $n$, and therefore $S$ contains three different points of a same line segment, but this again contradicts the strict convexity of $\|$.$\| . Therefore \left[x_{k, \Lambda}, x_{k, \Lambda}+w\right]$ is a maximal line segment in $S_{k}^{I d}$. 
By Proposition 4 (6) applied to $\|\cdot\|_{\lambda_{k}, I d}$, we therefore deduce that $b_{k} \geq\|w\| \geq$ $\lambda_{k}^{-1}-1$, which proves $\left(6^{\prime}\right)$ and concludes the proof.

We shall say that a vector $x \in X$ separates a bounded group $G$ of isomorphisms on $X$ (or sometimes that $X$ is separating for $G$ ) if for any $g \neq h$ in $G, g x \neq h x$, or equivalently, $g x \neq x$ whenever $g \neq I d$. Saying that $x$ separates $G$ and that the orbit $G x$ is discrete is easily equivalent to inf $g \neq I d\|x-g x\|>0$.

We shall now use Proposition 6 to renorm a separable real space $(X,\|\|$.$) with an$ equivalent norm $\||\cdot \||$ for which the group of isometries on $X$ is some given group $G$ of $\|$.$\| -isometries. This will of course depend on certain conditions on G$ and $X$. We begin with a lemma.

Lemma 7. Let $X$ be a separable real Banach space with a norm $\|$.$\| and let G$ be a countable group of isometries on $X$ such that $-I d \in G$. Assume that there exists a normalized vector $x_{0}$ in $X$ which separates $G$ and such that the orbit $G x_{0}$ is discrete. Let $\alpha \in] 0, \inf _{g \neq I d}\left\|x_{0}-g x_{0}\right\|[$. Then there exists a countable set $K$ containing 0 , a partition $\left(\{0\}, T_{1}, T_{2}\right)$ of $K$, and points $\left(x_{k}\right)_{k \in K}$ in the unit sphere of $X$ such that

(a) If $k \in K$, then $x_{k}=a_{k} x_{0}+z_{k}$ with $a_{k}>0$ and $\left\|z_{k}\right\| \in[\alpha / 10, \alpha / 5]$.

(b) The space $X$ is the closed linear span of the set $\left\{g x_{k}: g \in G, k \in\{0\} \cup T_{1}\right\}$.

(c) If $k \in T_{2}$, then $x_{k}=a_{k} x_{0}+\alpha_{k} g_{k} x_{0}$, with $a_{k}>0$ and $\alpha_{k} \in[\alpha / 10, \alpha / 5]$, $g_{k} \in G \backslash\{-I d, I d\}$.

(d) For all $k \in K, \inf \left\{\left\|x_{j}-g x_{k}\right\|: j \in K, g \in G,(j, g) \neq(k, I d)\right\}>0$.

Points $x_{k}$ for $k \in T_{1}$ will be said to be of type 1 , and points $x_{k}$ for $k \in T_{2}$ will be said to be of type 2 . The point $x_{0}$ will be the unique point of type 0 .

Proof. We first define a finite or infinite sequence of vectors $\left(y_{n}\right)_{n \in N}$ by induction. Let $V_{0}=\overline{\operatorname{span}}\left\{g x_{0}, g \in G\right\}$ and let $y_{0}=x_{0}$. If $V_{0}=X$, then we let $N=\{0\}$ and we are done. Otherwise we pick some $y_{1} \notin V_{0}$ and consider $V_{1}=\overline{\operatorname{span}}\left\{g y_{n}, n=\right.$ $0,1, g \in G\}$. If $V_{1}=X$, then we let $N=\{0,1\}$ and we are done. Otherwise, repeating the procedure, it is possible to pick a finite or infinite sequence $\left(y_{n}\right)_{n \in N}$ such that, if $V_{n}:=\overline{\operatorname{span}}\left\{g y_{k}, k \leq n, g \in G\right\}$, we have that $y_{n} \notin V_{n-1}$ for all $n \geq 1$ and $\bigcup_{n \in N} V_{n}$ is dense in $X$.

We associate to $\left(y_{n}\right)_{n \in N}$ an enumeration $\left(u_{n}\right)_{n \in K}$ of $\left\{g x_{0}, g \in G \backslash\{ \pm I d\}\right\} \cup$ $\left\{y_{n}, n \in N\right\}$. Note that $K$ may be infinite (when $G$ is infinite or $X$ is infinite dimensional), in which case we shall assume $K=\mathbb{N}$, or finite (when $G$ is finite and $X$ is finite dimensional), in which case we shall assume $K=\{0,1, \ldots,|K|-1\}$. We shall also assume that the enumeration starts with $y_{0}$, i.e. $u_{0}=y_{0}=x_{0}$.

We let

$$
T_{1}=\left\{n \in K: u_{n}=y_{k} \text { for some } k \geq 1\right\}
$$

and

$$
T_{2}=\left\{n \in K: u_{n}=g x_{0} \text { for some } g \in G \backslash\{ \pm I d\}\right\} .
$$

Note that if $G=\{-I d, I d\}$, then $\left(u_{n}\right)$ is simply an enumeration of $\left(y_{n}\right)$, in which case $T_{2}=\emptyset$ and there will only be points of types 0 or 1 . Likewise, when $X=$ $\overline{\operatorname{span}}\left\{g x_{0}, g \in G\right\}$, then $T_{1}=\emptyset$ and there will only be points of types 0 or 2 . If $T_{1}=T_{2}=\emptyset$, then we are in the extreme (and simplest) case when $G=\{-I d, I d\}$ and $X$ is one dimensional, and the result is trivial, but the following proof is still valid. 
Associated to $\left(u_{n}\right)_{n \in K}$ we define a sequence $\left(x_{n}\right)_{n \in K}$ of normalized vectors of $X$ as follows. For $n=0, x_{0}$ is already defined, and, since $x_{0}=(1-\alpha / 10) x_{0}+(\alpha / 10) x_{0}$, satisfies (a).

The definition of $x_{n}$ 's of type 1 , that is for $n \in T_{1}$, is as follows. For any such $n$, let $k \geq 1$ be such that $u_{n}=y_{k}$ and let $E_{k}=\operatorname{span}\left(V_{k-1}, y_{k}\right)$. Pick some $z_{n} \in E_{k}$ such that $\left\|z_{n}\right\| \in[\alpha / 10, \alpha / 5]$ and $d\left(z_{n}, V_{k-1}\right)=\alpha / 10$, and let $x_{n}=a_{n} x_{0}+z_{n}$, where $a_{n}>0$ is such that $\left\|x_{n}\right\|=1$. Obviously (a) is satisfied for such $x_{n}$ 's. To prove property (b), observe that $V_{0} \subset \overline{\operatorname{span}}\left\{g x_{0}, g \in G\right\}$, and that if $V_{k-1} \subset$ $\overline{\text { span }}\left\{g x_{n}, g \in G, n \in\{0\} \cup T_{1}: u_{n}=y_{j}\right.$ for some $\left.j \leq k-1\right\}$, then

$$
V_{k} \subset \overline{V_{k-1} \oplus \operatorname{span}\left\{g y_{k}, g \in G\right\}} \subset \overline{V_{k-1} \oplus \operatorname{span}\left\{g x_{n}, g \in G\right\}},
$$

where $n \in T_{1}$ is such that $u_{n}=y_{k}$, since $V_{k-1} \oplus \operatorname{span} y_{k}=E_{k}=V_{k-1} \oplus \operatorname{span} x_{n}$ and since $V_{k-1} \oplus \operatorname{span} g y_{k}=g E_{k}=V_{k-1} \oplus \operatorname{span} g x_{n}$. Therefore by induction,

$$
V_{k} \subset \overline{\operatorname{span}}\left\{g x_{n}, g \in G, n \in\{0\} \cup T_{1}: u_{n}=y_{j} \text { for some } j \leq k\right\},
$$

and since $\bigcup_{k} V_{k}$ is dense in $X$, it follows that

$$
X=\overline{\operatorname{span}}\left\{g x_{n}, g \in G, n \in\{0\} \cup T_{1}\right\} .
$$

The definition of $x_{n}$ 's of type 2, that is for $n \in T_{2}$, is more refined and will require an induction. For any such $n$, that is if $u_{n}$ is of the form $g_{n} x_{0}$ for $g_{n} \in G$, we shall define $x_{n}$ as follows: we shall pick some $\alpha_{n} \in[\alpha / 10, \alpha / 5]$ and define $z_{n}=\alpha_{n} g_{n} x_{0}$, $x_{n}=a_{n} x_{0}+z_{n}$ with $a_{n}>0$ and $\left\|x_{n}\right\|=1$. That is, (c) simply states the definition of such points, and assertion (a) follows immediately for points of type 2 .

To conclude the proof of the lemma, it only remains to check that the points $\left(x_{n}\right)$ may be chosen in such a way as to ensure that condition (d) is satisfied. The difficult part is to verify condition (d) for points of type 2, and for this the choice of $\alpha_{n}$ in their definition will need to follow a precise procedure. Before writing this procedure, which is based on an induction on $n \in T_{2}$, we shall note a few estimates which only require knowing that $\alpha_{n} \in[\alpha / 10, \alpha / 5]$ for such $n$ 's. First of all it will be useful to observe that for all $n,\left\|z_{n}\right\| \leq \alpha / 5$ and therefore $a_{n} \in[1-\alpha / 5,1+\alpha / 5]$. This holds for $x_{0}$ as well as for points of types 1 or 2 .

Therefore our aim is to obtain lower estimates for expressions of the form $\left\|x_{n}-g x_{m}\right\|$, when $(n, I d) \neq(m, g)$. We distinguish four cases.

First case. $g \neq I d$.

If $g \neq I d$, then $\left\|x_{n}-g x_{m}\right\|=\left\|a_{n} x_{0}+z_{n}-g a_{m} x_{0}-g z_{m}\right\| ;$ therefore

$$
\left\|x_{n}-g x_{m}\right\| \geq\left\|x_{0}-g x_{0}\right\|-\left|1-a_{n}\right|-\left|1-a_{m}\right|-\left\|z_{n}\right\|-\left\|g z_{m}\right\| \geq \alpha / 5 .
$$

Second case. $g=I d$ and $x_{\max (m, n)}$ is of type 1 .

Without loss of generality assume $n>m$. Since $x_{n}$ is of type 1 , if $k$ is such that $x_{n}$ is associated to $y_{k}$, the vector $x_{m}$ is in $V_{k-1}$ and so

$$
\left\|x_{n}-x_{m}\right\| \geq d\left(x_{n}, V_{k-1}\right)=\alpha / 10 .
$$

Third case. $g=I d, x_{\max (m, n)}$ is of type $2, x_{\min (m, n)}$ is of type 1 .

Assuming $n>m$, since $x_{n}$ is of type 2 and $x_{m}$ is of type 1 , then

$$
\left\|x_{n}-x_{m}\right\| \geq d\left(x_{m}, V_{0}\right) \geq \alpha / 10 .
$$

Fourth case. $g=I d, x_{\max (m, n)}$ is of type $2, x_{\min (m, n)}$ is of type 0 or 2. 
We describe the induction we need to choose the $x_{n}$ 's of type 2 ; i.e., we describe how to choose each corresponding $\alpha_{n} \in[\alpha / 10, \alpha / 5]$ in the definition of $x_{n}, n \in T_{2}$ to obtain good estimates for $\left\|x_{n}-x_{m}\right\|$ in this fourth case.

Let $T_{2}=\left\{m_{1}, \ldots, m_{\left|T_{2}\right|}\right\}$, when $G$ is finite, or $T_{2}=\left\{m_{1}, m_{2}, \ldots\right\}$, when $G$ is infinite, be the increasing enumeration of $T_{2}$, and let $m_{0}=0$. For $m \in T_{2}$, recall that $g_{m}$ denotes the element of $G$ such that $u_{m}=g_{m} x_{0}$, and that the corresponding $x_{m}$ will have the form $a_{m} x_{0}+\alpha_{m} g_{m} x_{0}$.

For every $m \in T_{2}$, let $I_{m}^{0}=[\alpha / 10, \alpha / 5]$. For $\beta \in[\alpha / 10, \alpha / 5]$, let $x_{m}(\beta)=$ $a_{m}(\beta) x_{0}+\beta g_{m} x_{0}$, where $b_{m}(\beta)>0$ is such that $\left\|x_{m}(\beta)\right\|=1$. For each $m \in T_{2}$ we shall pick some $\beta_{m} \in[\alpha / 10, \alpha / 5]$ such that $x_{m}=x_{m}\left(\beta_{m}\right)$ is a good choice for the corresponding point of type 2 .

We observe that $\left\|x_{m}(\beta)-x_{m}(\gamma)\right\| \geq \frac{\alpha}{2}|\beta-\gamma|$, for all $\beta$, $\gamma$ in $[\alpha / 10, \alpha / 5]$. Indeed if $x_{m}(\beta)-x_{m}(\gamma)=(\beta-\gamma) \epsilon$ with $\|\epsilon\|<\alpha / 2$ and $\beta \neq \gamma$, then by the definition of $x_{m}(\beta)$ and $x_{m}(\gamma)$,

$$
\left(b_{m}(\beta)-b_{m}(\gamma)\right) x_{0}=(\gamma-\beta)\left(g_{m} x_{0}-\epsilon\right),
$$

so $g_{m} x_{0}-\epsilon= \pm\left\|g_{m} x_{0}-\epsilon\right\| x_{0}$. If for example $\pm=-$ in this equality, then since

$$
\left|1-\left\|g_{m} x_{0}-\epsilon\right\|\right|=\left|\left\|g_{m} x_{0}\right\|-\left\|g_{m} x_{0}-\epsilon\right\|\right| \leq\|\epsilon\|,
$$

it follows that

$$
\left\|g_{m} x_{0}+x_{0}\right\|=\left\|\epsilon+x_{0}-\right\| g_{m} x_{0}-\epsilon\left\|x_{0}\right\| \leq\|\epsilon\|+\left\|x_{0}\right\|\|\epsilon\|<\alpha,
$$

and by definition of $\alpha, g_{m}=-I d$, a contradiction. Similarly the case $\pm=+$ would imply $g_{m}=I d$.

Now for all $m \in T_{2}$, divide $I_{m}^{0}=[\alpha / 10, \alpha / 5]$ into three successive intervals of equal length $\alpha / 30$. Since

$$
\left\|x_{m}(\beta)-x_{m}(\gamma)\right\| \geq \frac{\alpha}{2}|\beta-\gamma| \geq \frac{\alpha^{2}}{60}
$$

whenever $\beta$ is in the first and $\gamma$ in the last interval, it follows that there exists an interval $I_{m}^{1} \subset I_{m}^{0}$ of length $\alpha / 30$ (which is either the first or the last subinterval), such that

$$
\beta \in I_{m}^{1} \Rightarrow\left\|x_{m}(\beta)-x_{0}\right\| \geq \frac{\alpha^{2}}{120} .
$$

We then pick $\beta_{m_{1}}$ in $I_{m_{1}}^{1}$ and fix $x_{m_{1}}=x_{m_{1}}\left(\beta_{m_{1}}\right)$. Therefore we have ensured that

$$
\left\|x_{m_{1}}-x_{0}\right\| \geq \frac{\alpha^{2}}{120} \text {. }
$$

Given $n \in \mathbb{N}, n \geq 2$, assume that for all $m \in K_{2}$ such that $m<m_{n}$, some $\beta_{m}$ and $x_{m}=x_{m}\left(\beta_{m}\right)$ have been selected, as well as for any $1 \leq k \leq n-1$ and $m \in K_{2}$ such that $m \geq m_{k}$, an interval $I_{m}^{k}$ of length $\frac{\alpha}{10.3^{k}}$, in such a way that

- $I_{m}^{i} \subset I_{m}^{j}$ if $m \geq m_{i}$ and $i \geq j$.

- Whenever $0 \leq k<n-1, m \geq m_{k}$ and $\beta \in I_{m}^{k}$,

$$
\left\|x_{m}(\beta)-x_{m_{k}}\right\| \geq \frac{\alpha^{2}}{40.3^{k+1}} .
$$

Then for any $m \in T_{2}, m \geq m_{n-1}$, dividing $I_{m}^{n-1}$ into three subintervals and picking the first or the last, we find by the same reasoning as above some $I_{m}^{n} \subset I_{m}^{n-1}$ of length $\frac{\alpha}{10.3^{n}}$ with

$$
\beta \in I_{m}^{n} \Rightarrow\left\|x_{m}(\beta)-x_{m_{n-1}}\right\| \geq \frac{\alpha^{2}}{40.3^{n}} .
$$


We then pick $\beta_{m_{n}}$ in $I_{m_{n}}^{n}$ and fix $x_{m_{n}}=x_{m_{n}}\left(\beta_{m_{n}}\right)$. So for all $0 \leq k \leq n-1$, $\beta_{m_{n}} \in I_{m_{n}}^{n} \subset I_{m_{n}}^{k+1}$ and therefore we have ensured that for any $0 \leq k<n$,

$$
\left\|x_{m_{n}}-x_{m_{k}}\right\| \geq \frac{\alpha^{2}}{40.3^{k+1}} .
$$

This easily implies that for any $k \neq n$,

$$
\left\|x_{m_{n}}-x_{m_{k}}\right\| \geq \frac{\alpha^{2}}{40.3^{k+1}},
$$

which concludes the fourth case.

Summing up the four cases, we obtain the following estimates. For $x_{0}$ we have that

$$
\inf \left\{\left\|x_{0}-g x_{m}\right\|,(m, g) \neq(0, I d)\right\} \geq \min \left(\alpha / 5, \alpha / 10, \alpha^{2} / 120\right)=\alpha^{2} / 120 .
$$

If $x_{n}$ is of type 1 , then

$$
\inf \left\{\left\|x_{n}-g x_{m}\right\|,(m, g) \neq(n, I d)\right\} \geq \min (\alpha / 5, \alpha / 10)=\alpha / 10 .
$$

If $x_{n}$ is of type 2 , and $k$ is such that $n=m_{k}$, then

$$
\inf \left\{\left\|x_{n}-g x_{m}\right\|,(m, g) \neq(n, I d)\right\} \geq \min \left(\alpha / 10, \frac{\alpha^{2}}{40.3^{k+1}}\right)=\frac{\alpha^{2}}{40.3^{k+1}} .
$$

We have therefore finally proved (d), and this ends the proof of the lemma.

Theorem 8. Let $X$ be a separable real Banach space with an LUR-norm $\|$.$\| and$ let $G$ be a countable group of isometries on $X$ such that $-I d \in G$. Assume that there exists a normalized vector $x_{0}$ in $X$ which separates $G$ and such that the orbit $G x_{0}$ is discrete. Then $X$ admits an equivalent norm $\||\cdot \||$ such that $G$ is the group of isometries on $X$ for $\|\mid$.$\| .$

Proof. Since $G x_{0}$ is discrete and $x_{0}$ separates $G$, let $\left.\alpha \in\right] 0,1[$ be such that $\left\|x_{0}-g x_{0}\right\| \geq \alpha$, for all $g \neq I d$. Then Lemma 7 applies, so let $\left(x_{n}\right)_{n \in K}$ be the associated family of points. Because of Lemma 7 (d),

$$
c_{k}:=\inf \left\{\left\|x_{j}-g x_{k}\right\|: j \in K, g \in G,(j, g) \neq(k, I d)\right\}
$$

is positive for each $k \in K$. Therefore (2) in Proposition [6 is satisfied, and (1) is clearly satisfied since $\|\cdot\|$ is LUR and therefore strictly convex.

We let $\delta_{k}=c_{k} / 3$ for each $k$ and define sequences $b_{k}$ and $\lambda_{k}$ by induction as follows. Let $b_{1}=1$. Let $\lambda_{0,1}$ be given by Proposition 6 for $m=1 / 2, c_{1}, \delta_{1}$, and $b_{1}$, and pick $\lambda_{1}$ in the interval ] $\lambda_{1,1}, 1$ [. Given $b_{k}$ and $\lambda_{k}$, let $\delta_{k+1}=c_{k+1} / 4$ and let $b_{k+1}$ satisfy $b_{k+1}<\min \left(b_{k},\left(\lambda_{k}^{-1}-1\right) / 2\right)$. We let $\lambda_{0, k+1}$ be the number given by Proposition 6 for $m=1 / 2$ and $c_{i}, \delta_{i}, b_{i}, 1 \leq i \leq k+1$. We fix some $\lambda_{k+1}>\max \left(\lambda_{0, k+1}, \lambda_{k}\right)$.

We then define $\||\cdot|\|$ as the $(\Lambda, G)$-pimple at $\left(x_{n}\right)_{n}$ for $\Lambda=\left(\lambda_{n}\right)_{n}$. Therefore Proposition 6] applies.

Observe that $E=\left\{\lambda_{n}^{-1} g x_{n}, g \in G, n \in K\right\}$ is the set of isolated extremal points of $\left\||. \||\right.$. Indeed for a point $x$ of $S_{\Lambda, G}$ either $\| x /\|x\|-g x_{k} \|<c_{k} / 2$ for some $g, k$, in which case by $\left(5^{\prime}\right) x=\lambda_{k}^{-1} g x_{k}$ if it is an isolated extremal point; or $\|x /\| x\left\|-g x_{k}\right\| \geq c_{k} / 2>\delta_{k}$ for all $g, k$. Then by $\left(4^{\prime}\right),\|\|=.\||\cdot \||$ in a neighborhood of $x$ and then $x$ is not an isolated extremal point since $\|\cdot\|$ is LUR at $x$.

Therefore any isometry $T$ for $\||\cdot \||$ maps $E$ onto itself. If $n<m$ and $g \in G$, then $T$ cannot map $\lambda_{n}^{-1} x_{n}$ to $\lambda_{m}^{-1} g x_{m}$. Indeed if $w$ (resp. $\left.w^{\prime}\right)$ is a vector so that $\lambda_{n}^{-1} x_{n}$ 
and $\lambda_{n}^{-1} x_{n}+w\left(\right.$ resp. $\lambda_{m}^{-1} g x_{m}$ and $\left.\lambda_{m}^{-1} g x_{m}+w^{\prime}\right)$ are endpoints of a maximal line segment in the unit sphere of $\||\cdot \||$, then since $g$ is an isometry for $\||\cdot \||$ we may assume $g=I d$, and then by $\left(3^{\prime}\right)$ and $\left(6^{\prime}\right)$,

$$
\left\|\left|w\left\|\mid \geq \frac{1}{2}\right\| w\left\|\geq \frac{1}{2}\left(\lambda_{n}^{-1}-1\right)>b_{n+1} \geq b_{m} \geq\right\| w^{\prime}\|\geq\| w^{\prime} \| .\right.\right.
$$

Likewise, if $n>m$, we may by using $T^{-1}$ deduce that $T$ cannot map $\lambda_{n}^{-1} x_{n}$ to $\lambda_{m}^{-1} x_{m}$.

Finally it follows that for each $n$, the orbit $G x_{n}$ is preserved by $T$.

We finally prove that $T$ belongs necessarily to $G$. Without loss of generality, we may assume that $T x_{0}=x_{0}$ and then by Lemma 7 (b) it is enough to prove that $T g x_{n}=g x_{n}$ for all $g \in G$ and any $x_{n}$ of type 1 or equal to $x_{0}$.

Let $g \in G, g \neq \pm I d$. Let $x_{k}$ be the associated vector of type 2 of the form $x_{k}=a x_{0}+\beta g x_{0}$ given by Lemma 7 (c). Then since $T x_{k}=h x_{k}$ for some $h \in G$,

$$
T x_{k}=a x_{0}+\beta T g x_{0}=h\left(a x_{0}+\beta g x_{0}\right) .
$$

So $|a|\left\|x_{0}-h x_{0}\right\|=\beta\left\|T g x_{0}-h g x_{0}\right\|$ and

$$
\begin{gathered}
\left.\left\|x_{0}-h x_{0}\right\| \leq \frac{\alpha / 5}{1-\alpha / 5}\left(\left\|h g x_{0}\right\|+\left\|T g x_{0}\right\|\right)\right) \leq \frac{\alpha / 5}{1-\alpha / 5}\left(\left\|x_{0}\right\|+2\left\|\mid T g x_{0}\right\| \|\right) \\
\leq \frac{\alpha}{4}\left(1+2\left\|\left|x_{0} \|\right|\right)<\alpha\right.
\end{gathered}
$$

therefore by the definition of $\alpha, h=I d$. It follows immediately from the expression of $T x_{k}$ that

$$
T g x_{0}=g x_{0},
$$

and this holds for any $g \in G$. Now let $x_{n}$ be of type 1 , of the form $a_{n} x_{0}+z_{n}$, according to Lemma 8 (a), and let $g \in G$. Then

$$
T g x_{n}=T\left(a_{n} g x_{0}+g z_{n}\right)=a_{n} g x_{0}+T g z_{n},
$$

and since $T\left(g x_{n}\right)$ is of the form $h x_{n}$ for some $h \in G$, we also have

$$
T g x_{n}=a_{n} h x_{0}+h z_{n} .
$$

Therefore

$$
a_{n} g x_{0}+T g z_{n}=a_{n} h x_{0}+h z_{n}
$$

so $a_{n}\left\|g x_{0}-h x_{0}\right\|=\left\|T g z_{n}-h z_{n}\right\|$ and therefore,

$$
\left\|g x_{0}-h x_{0}\right\| \leq \frac{\left\|h z_{n}\right\|+\left\|T g z_{n}\right\|}{1-\alpha / 5} \leq \frac{\alpha / 5+2\left\|\left|T g z_{n} \|\right|\right.}{1-\alpha / 5} \leq \frac{3 \alpha / 5}{1-\alpha / 5}<\alpha,
$$

whence $g=h$ and

$$
\operatorname{Tg} x_{n}=g x_{n} .
$$

Finally since $T g x_{n}=g x_{n}$ for any $g \in G$ and $x_{n}$ equal to $x_{0}$ or of type 1 , Lemma 7 (b) implies that $T=I d$ and therefore belongs to $G$. 
2.2. Representable groups of linear isomorphisms. In this subsection, we give sufficient conditions for a group of isomorphisms on a Banach space $X$ to be representable in $X$. We shall need a well-known result of Kadec about LURrenormings; see [4, Theorem 2.6, p.48.

Theorem 9 (Kadec, 1965). Any separable real Banach space admits an equivalent LUR norm.

We shall also use a refinement obtained by G. Lancien for spaces with the RadonNikodym Property; see [14, Theorem 2.1. The fact concerning the isometries in this result is not written explicitly in 14 but is obvious from the definition of the renorming. For the exact definition of the Radon-Nikodym Property we refer to [15. In our applications we shall only use the fact that any separable dual space has the Radon-Nikodym Property.

Theorem 10 (Lancien, 1993). Any separable real Banach space with the RadonNikodym Property may be renormed with an equivalent LUR norm without diminishing the group of isometries.

Using Theorem 9 we obtain:

Theorem 11. Let $X$ be a separable real Banach space and let $G$ be a finite group of isomorphisms such that $-I d \in G$. Then $X$ admits an equivalent norm for which $G$ is the group of isometries on $X$.

Proof. By Theorem 9 we may assume that the norm $\|$.$\| on X$ is LUR. Then we define an equivalent norm $\|\cdot\|_{G}$ on $X$ by

$$
\|x\|_{G}=\left(\sum_{g \in G}\|g x\|^{2}\right)^{1 / 2} .
$$

Since this is the $\ell_{2}$-sum of the LUR norm $\|$. $\|$ with an equivalent norm, it is classical to check that it is also LUR, see [4] Fact 2.3, and obviously any $g \in G$ becomes an isometry for $\|\cdot\|_{G}$. To apply Theorem 8 and since $G$ is finite, it therefore only remains to find some $x_{0}$ such that $x_{0} \neq g x_{0}$ for all $g \neq I d$. But if such an $x_{0}$ didn't exist, then we would have that $X=\bigcup_{g \in G} \operatorname{Ker}(I d-g)$, and $\operatorname{Ker}(I d-g)$ would have nonempty interior for some $g \neq I d$, which would contradict the fact that no proper subspace of $X$ can have nonempty interior.

We use Theorem 10 to obtain:

Theorem 12. Let $X$ be a separable real Banach space with the Radon-Nikodym Property and let $G$ be a countable bounded group of isomorphisms on $X$, containing $-I d$, and such that some point separates $G$ and has discrete orbit. Then $X$ admits an equivalent norm for which $G$ is the group of isometries on $X$.

Proof. We may assume that every $g$ in $G$ is an isometry on $X$ by using the equivalent norm $\sup _{g \in G}\|g x\|$. Then by Theorem 10, $X$ may be renormed with an LUR norm without diminishing the group of isometries, and therefore any $g \in G$ is still an isometry in the new norm. We are then in a position to apply Theorem 8

It is natural to ask in what degree the condition of the existence of a separating point with discrete orbit in Theorem 12 is necessary. To investigate this question we give the following definitions. 
We denote by $L(X)$ the space of bounded linear operators on $X$. We shall denote by $s$ the strong operator topology on $L(X)$; that is, a sequence $T_{n}$ converges to $T$ for $s$ if and only if $T_{n} x$ converges to $T x$ for any $x \in X$. For $c>0$, we denote by $L_{c}(X)$ the set of operators of norm less than or equal to $c$. We shall also consider the relative strong operator topology on $L_{c}(X)$, which is a closed subset of $L(X)$ for $s$. It is a fact, proved in [13], page 14, that when $X$ is separable, $\left(L_{c}(X), s\right)$ is Polish (that is, separable metrizable complete).

For $c \geq 1$, we denote by $I_{c}(X)$ the set of isomorphisms $T$ on $X$ such that $T \in L_{c}(X)$ and $T^{-1} \in L_{c}(X)$. We shall denote by $t$ the topology of the strong convergence of $T$ and $T^{-1}$ on $I_{c}(X)$ : that is, a sequence $T_{n}$ converges to $T$ if and only if $T_{n}$ converges to $T$ and $T_{n}^{-1}$ to $T^{-1}$ in the strong operator topology. In other words, this is the topology induced by the representation of $I_{c}(X)$ as a subspace of $\left(L_{c}(X), s\right) \times\left(L_{c}(X), s\right)$ through the map $\phi(T)=\left(T, T^{-1}\right)$.

Let $G$ be a bounded group of isomorphisms satisfying the condition in Theorem 12 that some point $x_{0}$ separates $G$ and has discrete orbit; that is, there exists $\alpha>0$ such that $\left\|x_{0}-g x_{0}\right\| \geq \alpha$ for all $g \in G$. Then $G$ is easily seen to be closed and discrete in $L(X)$ with the strong operator topology, and also closed and discrete in $I_{c}(X)$ for the topology of the strong convergence of $T$ and $T^{-1}$, whenever $c \geq \sup _{g \in G}\|g\|$.

We shall now see that this last condition is actually necessary. Indeed, conversely to Theorem 12 .

Lemma 13. Let $X$ be a separable real Banach space and let $G$ be a countable group of isomorphisms which is the group of isometries in some equivalent norm on $X$. Then $G$ is closed and discrete for the topology of the strong convergence of $T$ and $T^{-1}$, and $G$ admits a separating point. If $X$ is finite dimensional, then $G$ is finite.

Proof. The existence of a separating point for $G$ is a consequence of the Theorem of Baire. Indeed for any $g \in G, g \neq I d$, the set of points which separate $g$ from $I d$, i.e. the set $X \backslash \operatorname{Ker}(g-I d)$, is dense open. Therefore the set of separating points is a $G_{\delta}$ dense set.

We now fix $c \geq \sup _{g \in G}\|g\|$, so that $G \subset I_{c}(X)$, and claim that $G$ is a closed subset of $I_{c}(X)$. This is equivalent to saying that $\phi(G)$ is a closed subset of $\phi\left(I_{c}(X)\right)$, and to prove this, it is enough to prove that $\phi(G)$ is a closed subset of $L_{c}(X) \times L_{c}(X)$. To see this observe that if $g_{n}$ converges to $T$ and $g_{n}^{-1}$ to $U$ in $\left(L_{c}(X), s\right)$, then

$$
\|T U x-x\| \leq\left\|\left(T-g_{n}\right) U x\right\|+\left\|g_{n}\right\|\left\|\left(U-g_{n}^{-1}\right) x\right\|,
$$

which converges to 0 when $n$ tends to $+\infty$. So $T U x=x$ and, in the same manner, $U T x=x$. Furthermore

$$
\left\|\left|T x \left\|\left|=\lim _{n}\left\|\left|g_{n} x\||=\||x \||\right.\right.\right.\right.\right.\right.
$$

in the equivalent norm $\||\|| \mid$ for which $G$ is the group of isometries on $X$, so $T$ and $U$ are isometric injections for that norm, and therefore surjective isometries such that $U=T^{-1}$. This means that $T$ belongs to $G$, and $(T, U)$ belongs to $\phi(G)$. Finally $\phi(G)$ is closed, and in particular $G$ is closed in $I_{c}(X)$.

Next we prove that $G$ is discrete for $t$. Indeed since $X$ is separable, the ball $L_{c}(X)$ of $L(X)$ with the relative strong topology is Polish, and $L_{c}(X) \times L_{c}(X)$ is Polish as well. Since $\phi(G)$ is closed, it is Polish too. Now every countable Polish group is a discrete space. Indeed whenever $H$ is a countable Polish group, then by [13, Theorem $6.2, H$ is not a perfect space; that is, $H$ has an isolated point. 
Therefore by the group property all points are isolated. Therefore $\phi(G)$ is discrete, which means that $G$ is discrete for $t$.

Finally we assume that $X$ is finite dimensional and prove that $G$ must be finite. Since $\operatorname{dim} X<+\infty$, the strong operator topology on $L_{c}(X)$ coincides with the usual one for which $L_{c}(X)$ is compact. Since $\phi(G)$ is a closed subset of $L_{c}(X) \times L_{c}(X)$, it is also compact. So $\phi(G)$ as a discrete compact space is finite, and $G$ is finite.

The following question remains open.

Question 14. Is it true that for any real Banach space $X$, and any countable group $G$ of isomorphisms on $X$ which is the group of isometries on $X$ in some equivalent norm, there must exist a separating point for $G$ with discrete orbit?

It is also natural to wonder whether the only role of the separation and discrete orbit hypothesis in Theorem 12 is to guarantee the closedness of the group $G$. That is, for groups which are not closed for the convergence of $T$ and $T^{-1}$ in the strong operator topology, one may wish to generalize Theorem 12 by showing that whenever $X$ is separable with the RNP, and $G$ is a countable bounded group of isomorphisms containing $-I d$, then there exists an equivalent norm for which the group of isometries is equal to the corresponding closure $\bar{G}^{s}$. This however is false, as proved by the next example.

Example 15. Let $G$ be the group of rational rotations on $\mathbb{C}$. Then $\mathbb{C}$ cannot be renormed, as a real space, so that the group of $\mathbb{R}$-linear isometries on $X$ is $\bar{G}^{s}$.

Proof. The set $\bar{G}^{s}$ is the set of rotations on $\mathbb{C}$. If $\||\cdot \||$ is a new real norm on $\mathbb{C}$ which is invariant by rotations, then it is a multiple of the modulus. But then the symmetry with respect to the real axis is an isometry on $\mathbb{C}$ which does not belong to $\bar{G}^{s}$.

The next question also remains open for spaces which do not satisfy the RNP.

Question 16. Let $X$ be a separable real Banach space, let $G$ be an infinite countable bounded group of isomorphisms on $X$ such that $-I d \in G$, and suppose some point separates $G$ and has discrete orbit. Does $X$ admit an equivalent norm for which $G$ is the group of isometries on $X$ ?

2.3. Representation of countable groups in Banach spaces. Jarosz conjectured that any group of the form $\{-1,1\} \times G$ (or $\mathbb{C}_{1} \times G$ in the complex case) could be represented in any Banach space $X$ provided $\operatorname{dim} X \geq|G|$. From Theorem 11 and Theorem 8 we obtain rather general answers to his question for countable groups and separable real spaces.

Theorem 17. Let $G$ be a finite group and let $X$ be a separable real Banach space such that $\operatorname{dim} X \geq|G|$. Then $\{-1,1\} \times G$ is representable in $X$.

Proof. The group $\{-1,1\} \times G$ may be canonically represented as a group of isometries on $\ell_{2}(G)$ : denoting by $\left(e_{g}\right)_{g \in G}$ the canonical basis of $\ell_{2}(G)$, associate to any $(\epsilon, g)$ in $\{-1,1\} \times G$ the isometry $T_{\epsilon, g}$ defined on $\ell_{2}(G)$ by

$$
T_{\epsilon, g}\left(\sum_{h \in G} \lambda_{h} e_{h}\right)=\epsilon \sum_{h \in G} \lambda_{h} e_{g h} .
$$


Since $\operatorname{dim} X \geq|G|$, the space $X$ is isomorphic to the $\ell_{2}$ direct sum $\ell_{2}(G) \oplus_{2} Y$, for some space $Y$. By associating to any $(\epsilon, g)$ in $\{-1,1\} \times G$ the isometry $A_{\epsilon, g}$ defined on $\ell_{2}(G) \oplus_{2} Y$ by

$$
A_{\epsilon, g}(t, y)=\left(T_{\epsilon, g}(t), \epsilon y\right),
$$

we see that $\{-1,1\} \times G$ is isomorphic to a group of isometries on $\ell_{2}(G) \oplus_{2} Y$ containing $-I d=A_{-1, I d}$. Therefore Theorem 11 applies to deduce that $\{-1,1\} \times G$ is isomorphic to the group of isometries on $X$ in some equivalent norm.

By Lemma 13 an infinite countable group is representable in a real space $X$ only if $X$ is infinite dimensional. For finite groups, it is natural to ask whether the condition on the dimension is necessary in Theorem 17. This is not the case when $|G|$ is an odd prime. Indeed, letting $p=|G|, G$ is then isomorphic to $\mathbb{Z} / p \mathbb{Z}$ and so $\{-1,1\} \times G$ is isomorphic to $\mathbb{Z} / 2 p \mathbb{Z}$ and therefore may be represented as the group $\left\{e^{i k \pi / p} I d, 0 \leq k \leq 2 p-1\right\}$ of isometries on $\mathbb{C}$; so $\{-1,1\} \times G$ is representable in $\mathbb{R}^{2}$. For other values of $|G|$, the question remains open:

Question 18. For arbitrarily large $n \in \mathbb{N}$, does there exist a group $G$ with $|G|=n$, such that $\{-1,1\} \times G$ is representable in a separable real Banach space $X$ if and only if $\operatorname{dim} X \geq n$ ?

A group which is representable in a Banach space necessarily admits a normal subgroup with two elements. We shall now see that to extend Theorem 17 to the case of groups which admit a normal subgroup with two elements, we shall need additional structure on the space. Recall that any (resp. countable) group which admits a normal subgroup with two elements is representable in a (resp. the separable) Hilbert space [19. The next theorem shows that this extends to a wide class of spaces, including the classical spaces $c_{0}, C([0,1]), \ell_{p}, 1 \leq p<+\infty$, and $L_{p}, 1 \leq p<+\infty$.

Recall that a Schauder basis $\left(s_{n}\right)$ of a Banach space $S$ is said to be $(C$ - $)$ symmetric, $C \geq 1$, when for any permutation $\sigma$ on $\mathbb{N}$, the sequence $\left(s_{\sigma(n)}\right)$ is $(C-)$ equivalent to $\left(s_{n}\right)$. The equivalent norm

$$
\left\|\left|\sum_{n} a_{n} s_{n}\left\|\mid=\sup _{\left|\lambda_{n}\right| \leq 1, \sigma}\right\| \sum_{n} \lambda_{n} a_{n} s_{\sigma(n)} \|\right.\right.
$$

then turns $\left(s_{n}\right)$ into a 1 -symmetric basis of $S$ such that the inequality $\left\|\left|\sum_{n} a_{n} s_{n} \|\right|\right.$ $\leq\left\|\left|\sum_{n} b_{n} s_{n} \|\right|\right.$ holds whenever $\left|a_{n}\right| \leq\left|b_{n}\right|$ for all $n$ (see [15]), in which case we shall say that it is standard symmetric.

When $S$ has a standard symmetric basis $\left(s_{n}\right)$, we denote by $\left(\sum \oplus Y\right)_{S}$ the space of sequences $\left(y_{n}\right)$ of elements of $Y$ such that $\sum_{n}\left\|y_{n}\right\| s_{n}$ converges, with the norm $\left\|\left(y_{n}\right)_{n}\right\|=\left\|\sum_{n}\right\| y_{n}\left\|s_{n}\right\|$. Note that the fact that $\left(s_{n}\right)$ is standard symmetric guarantees that this indeed defines a norm instead of a quasi-norm. We shall say that a Banach space has a symmetric decomposition if it is isomorphic to $\left(\sum \oplus Y\right)_{S}$, for some space $S$ with a standard symmetric basis $\left(s_{n}\right)_{n}$.

Although we shall only apply this definition in the special cases of a space with a symmetric basis, or of a space of the form $c_{0}(Y)$ or $\ell_{p}(Y)$, it is interesting to see that our results are more general and could apply to certain Orlicz-type sums for example. For a space of the form $X=\left(\sum \oplus Y\right)_{S}$, where $S$ has a standard symmetric basis $\left(s_{n}\right)_{n}$, we shall call standard isometry on $X$ any map $T$ defined on $X$ by $T\left(\left(y_{n}\right)_{n \in \mathbb{N}}\right)=\left(\epsilon_{n} y_{\sigma(n)}\right)_{n \in \mathbb{N}}$, where $\epsilon_{n}= \pm 1$ for all $n \in \mathbb{N}$ and $\sigma$ is a permutation on $\mathbb{N}$. Such a map is indeed isometric by the 1 -symmetry of $\left(s_{n}\right)_{n}$. 
Theorem 19. Let $G$ be a countable group which admits a normal subgroup with two elements and let $X$ be an infinite-dimensional separable real Banach space with a symmetric decomposition which either is isomorphic to $c_{0}(Y)$ or to $l_{p}(Y)$ for some $Y$ and $1 \leq p<+\infty$, or has the Radon-Nikodym Property. Then $G$ is representable in $X$.

Proof. We may assume that $X=\left(\sum \oplus Y\right)_{S}$, for some space $S$ with a standard symmetric basis $\left(s_{n}\right)_{n}$. We shall first prove that $G$ may be represented as some group of standard isometries on $X$ containing $-I d$ and for which there exists a separating vector with discrete orbit.

Let $\{1, j\}$ be a normal subgroup of $G$ with two elements; therefore $j$ commutes with any element of $G$. Let $G^{\prime}$ be a subset of $G$ containing one and exactly one element of each subset $\{g, j g\}$, so that $\left(G^{\prime}, j G^{\prime}\right)$ is a partition of $G$. For $g \in G$ let $\epsilon_{g}=1$ if $g \in G^{\prime}$ and $\epsilon_{g}=-1$ otherwise, and let $|g|$ denote the unique element of $\{g, j g\} \cap G^{\prime}$.

We first assume that $G$ is infinite. Then since $G^{\prime}$ is infinite, we may index the decomposition of $X$ on $G^{\prime}$, that is, write $X=\left(\sum \oplus Y\right)_{G^{\prime}}$ and any element of $X$ as $\left(y_{g}\right)_{g \in G^{\prime}}$. We associate to any $g$ in $G$ the standard isometry $T_{g}$ defined on $X$ by

$$
T_{g}\left(\left(y_{h}\right)_{h \in G^{\prime}}\right)=\left(\epsilon_{g^{-1} h} y_{\left|g^{-1} h\right|}\right)_{h \in G^{\prime}} .
$$

Observe that $T_{j}=-I d$ and that if $g, k \in G$, then

$$
T_{k} T_{g}\left(\left(y_{h}\right)_{h}\right)=T_{k}\left(\left(\epsilon_{g^{-1} h} y_{\left|g^{-1} h\right|}\right)_{h}\right)=\left(\epsilon_{k^{-1} h} \epsilon_{g^{-1}\left|k^{-1} h\right|} y_{\left|g^{-1}\right| k^{-1} h||}\right)_{h} .
$$

Since $j$ commutes with any element of $G$, we have $\left|g^{-1}\right| k^{-1} h||=\left|g^{-1} k^{-1} h\right|$ and it is easy to see that $\epsilon_{k^{-1} h} \epsilon_{g^{-1}\left|k^{-1} h\right|}=\epsilon_{g^{-1} k^{-1} h}$; therefore

$$
T_{k} T_{g}\left(\left(y_{h}\right)_{h}\right)=\left(\epsilon_{(k g)^{-1} h} y_{(k g)^{-1} h}\right)_{h}=T_{k g}\left(\left(y_{h}\right)_{h}\right) .
$$

From this we deduce that the map $g \mapsto T_{g}$ is a group isomorphism.

If $G$ is finite, then we enumerate $G^{\prime}$ as $\left\{g_{1}, \ldots, g_{\left|G^{\prime}\right|}\right\}$. By the same method as when $G^{\prime}$ is infinite we may represent $G$ as a group of isometries of the form $T\left(\left(y_{g}\right)_{g \in G^{\prime}}\right)=\left(\epsilon_{g} y_{\sigma(g)}\right)_{g \in G^{\prime}}$, where $\epsilon_{g}= \pm 1$ for all $g \in G^{\prime}$ and $\sigma$ is a permutation on $G^{\prime}$, on $Y^{\left|G^{\prime}\right|}$ equipped with the norm

$$
\left\|\left(y_{g}\right)_{g \in G^{\prime}}\right\|=\left\|\sum_{n \leq\left|G^{\prime}\right|}\right\| y_{g_{n}}\left\|s_{n}\right\| .
$$

For each $k=0,1, \ldots$ we let $G_{k}=\left\{k\left|G^{\prime}\right|, k\left|G^{\prime}\right|+1, \ldots, k\left|G^{\prime}\right|+k-1\right\}$ and note that the $G_{k}$ 's form a partition of $\mathbb{N}$. Since $\left|G_{k}\right|=\left|G^{\prime}\right|, G$ is also isomorphic to a group of isometries of the form $T\left(\left(y_{n}\right)_{n \in G_{k}}\right)=\left(\epsilon_{n} y_{\sigma(n)}\right)_{n \in G_{k}}$ on the subspace of $X$ spanned by the summands indexed in $G_{k}$. Let $g \mapsto T_{g}^{k}$ be the corresponding group isomorphism, and finally let $T_{g}$ be the standard isometry defined on $X$ by

$$
T_{g}\left(\left(y_{n}\right)_{n}\right)=\left(T_{g}^{0}\left(\left(y_{n}\right)_{n \in G_{0}}\right), T_{g}^{1}\left(\left(y_{n}\right)_{n \in G_{1}}\right), \ldots\right) .
$$

Since each $g \mapsto T_{g}^{k}$ is a group isomorphism, $g \mapsto T_{g}$ is a group isomorphism as well, and we also have $T_{j}=-I d$.

Finally we may therefore assume that $G$ is a group of standard isometries on $X$ containing $-I d$, whether $G$ is finite or infinite.

Now let $x_{0}$ be a normalized vector in the summand of the decomposition indexed by 1 . We observe that $\left\|x_{0}-\left(-x_{0}\right)\right\|=2$ and that for any $g \in G, g \notin\{-I d, I d\}$,

$$
\left\|x_{0}-g x_{0}\right\| \geq 1
$$


since the basis $\left(s_{g}\right)_{g \in G^{\prime}}$ of $S$ has basis constant 1 . Therefore $x_{0}$ separates $G$ and has discrete orbit. So we have represented $G$ as some group of isometries on $X$ containing $-I d$ and admitting a vector $x_{0}$ which separates $G$ and has discrete orbit.

To conclude we note that in the case when $X$ has the RNP, Theorem 12 applies, and $G$ is representable in $X$. When $X$ is isomorphic to $c_{0}(Y)$ or $\ell_{p}(Y)$ for some $1 \leq p<+\infty$, we could by Theorem 9 assume that $Y$ had an LUR norm. Then we may use two lemmas proved in the Appendix, Lemma 51 for $\ell_{p}(Y)$, Lemma 52 for $c_{0}(Y)$, to consider some equivalent LUR norm on $X$ for which all standard isometries in the original norm are still isometries in the new norm. In particular the $T_{g}$ 's are isometries on $X$ for this new norm, which implies that $G$ is representable as a group of isometries containing $-I d$ for the LUR norm on $X$. Since $x_{0}$ separates $G$ and has discrete orbit, Theorem 8 applies, and $G$ is representable in $X$.

Corollary 20. A countable group is representable in the real space $C([0,1])$, resp. $c_{0}, l_{p}$ for $1 \leq p<+\infty, L_{p}$ for $1 \leq p<+\infty$, if and only if it admits a normal subgroup with two elements.

Proof. $C([0,1]) \simeq c_{0}(C([0,1]))$ (see for example [18, p. 1553), $c_{0} \simeq c_{0}\left(c_{0}\right), \ell_{p} \simeq$ $\ell_{p}\left(\ell_{p}\right)$ and $L_{p} \simeq \ell_{p}\left(L_{p}\right)$ for $p \in[1,+\infty[$.

From Theorem 19 we may also deduce the following result.

Theorem 21. Let $G$ be a countable group and $X$ be an infinite-dimensional separable real Banach space which contains a complemented subspace with a symmetric basis. Then $\{-1,1\} \times G$ is representable in $X$.

Proof. The case when $G$ is finite comes from Theorem 17 so we may assume that $G$ is infinite. Let $Y$ be a complemented subspace of $X$ with a symmetric basis. Since a symmetric basis is unconditional, the classical theorem of James implies that $Y$ is either reflexive or contains a complemented subspace isomorphic to $c_{0}$ or $\ell_{1}$; therefore we may assume that $Y$ has the Radon-Nikodym Property or is isomorphic to $c_{0}$. By renorming we may therefore assume that either $Y$ has the $\mathrm{RNP}$ and a standard symmetric basis, or that $Y$ is isometric to $c_{0}$.

By Theorem 19 we may assume that $\{-1,1\} \times G$ is group isomorphic to the group of isometries on $Y$. Let $\alpha$ be the corresponding group isomorphism and note that by setting $j=(-1, I d)$ in the proof of Theorem 19 we may assume that $\alpha(-1, I d)=-I d_{Y}$.

Next we prove that $\alpha$ provides a group isomorphism of $\{-1,1\} \times G$ with some group of isometries on $Y$ for some equivalent LUR norm on $Y$. When $Y$ has the RNP this is a direct consequence of Theorem 10. When $Y$ is isometric to $c_{0}$, this is a consequence of the fact that the proof of Theorem 19 gives that $\alpha(\{-1,1\} \times G)$ is a group of standard isometries on $c_{0}$, and therefore that we may use Lemma 52 to see that $\alpha(\{-1,1\} \times G)$ is some group of isometries for some LUR norm on $c_{0}$. Note that in these two cases, by the proof of Theorem 19, the point $x_{0}=s_{1}$ separates $\{-1,1\} \times G$ and has discrete orbit, where $s_{1}$ is the first vector of the symmetric basis of $Y$.

Now write $X \simeq Y \oplus Z$, and note that since $Z$ is separable we may assume by Theorem 9 that it is equipped with an LUR norm. Then we equip $X$ with the 
equivalent $\ell_{2}$-sum norm $\left\||\cdot \||\right.$, corresponding to the $\ell_{2}$-sum $Y \oplus_{2} Z$. It is classical that the norm $\||\cdot \||$ is LUR on $X$ (see [4], Fact 2.3).

For any $(\epsilon, g)$ in $\{-1,1\} \times G$, the map $A_{\epsilon, g}$ defined on $X=Y \oplus_{2} Z$ by

$$
A_{\epsilon, g}(y, z)=(\alpha(\epsilon, g) . y, \epsilon z)
$$

is an isometry on $X$ for $\||\cdot \||$. Therefore $\{-1,1\} \times G$ is group isomorphic to a group of isometries on $\left(X,\||\cdot \||)\right.$ containing $-I d=A_{-1, I d}$. Since the point $x_{0}=s_{1}$ separates $\{-1,1\} \times G$ and has discrete orbit, Theorem 8 applies. This proves that $\{-1,1\} \times G$ is representable in $X$.

Observe that Theorem 21 applies whenever $X$ is a subspace of $\ell_{p}, 1 \leq p<+\infty$, or, by Sobczyk's Theorem, [15] Th. 2.f.5, whenever $X$ is separable and contains a copy of $c_{0}$.

Because of Theorem 19, it is natural to ask whether Theorem 17 and Theorem 21 extend to the case when one replaces groups of the form $\{-1,1\} \times G$ by groups which admit a normal subgroup with two elements. We provide examples to show that the answer is negative in general.

Recall that an operator $S$ from a space $Y$ into a space $X$ is strictly singular if no restriction of $S$ to an infinite-dimensional subspace of $Y$ is an isomorphism into. Equivalently, for any $\epsilon>0$ and any infinite-dimensional subspace $Z$ of $Y$, there exists a vector $y \in Z$ such that $\|S y\|<\epsilon\|y\|$. It is well known that the class $S(X)$ of strictly singular operators on $X$ is a closed two-sided ideal of $L(X)$ containing the compact operators.

We shall denote by $X_{G M}$ the real HI space of W.T. Gowers and B. Maurey [10, on which every operator $T$ is of the form $\lambda_{T} I d+S$, where $\lambda_{T} \in \mathbb{R}$, and $S$ is strictly singular. The ideal properties of $S(X)$ imply that, given $T \in L(X), \lambda_{T}$ is uniquely determined.

We observe that every isometry on $X_{G M}$ is of the form $\pm I d+S$. Indeed for any isometry $T$ on $X_{G M}$, and any $\epsilon>0$,

$$
|1-| \lambda_{T}|| \leq\left\|T y-\lambda_{T} y\right\|=\|S y\| \leq \epsilon,
$$

for some appropriate normalized $y \in X$. Actually we shall see in the last section that every isometry on $X_{G M}$ is of the form $\pm I d+K, K$ compact, but this refinement will not be needed here.

It will also be useful to consider the complex version of $X_{G M}$ [10, on which every $\mathbb{C}$-linear operator is of the form $\lambda I d+S, S$ strictly singular. For the same reasons as above, every $\mathbb{C}$-linear isometry on the complex version of $X_{G M}$ is of the form $\lambda_{T} I d+S$, with $\lambda_{T} \in \mathbb{C}_{1}$ and $S$ strictly singular (and actually, $S$ compact, by [17]), where again $\lambda_{T}$ is uniquely determined.

Proposition 22. Any group which is representable in the real (resp. the complex) $X_{G M}$ is of the form $\{-1,1\} \times G$ (resp. $\mathbb{C}_{1} \times G$ ). In particular a finite group is representable in the real $X_{G M}$ if and only if it is of the form $\{-1,1\} \times G$.

Proof. The last part of the proposition is a direct consequence of the initial part and of Theorem [17. We prove the initial part. Let $H$ be the group of $\mathbb{R}$-linear (resp. $\mathbb{C}$-linear) isometries on the real (resp. complex) $X_{G M}$ in some equivalent real (resp. complex) norm. Let $G$ be the subgroup of $H$ of isometries of the form $I d+S, S$ strictly singular. For $T \in H$, let $\lambda_{T}$ be the element of $\{-1,1\}$ (resp. 
$\mathbb{C}_{1}$ ) such that $T-\lambda_{T} I d$ is strictly singular. We consider the map $\alpha$ from $H$ onto $\{-1,1\} \times G\left(\right.$ resp. $\left.\mathbb{C}_{1} \times G\right)$ defined by

$$
\alpha(T)=\left(\lambda_{T}, T / \lambda_{T}\right) .
$$

It is then easy to see, using the ideal properties of strictly singular operators, that $\lambda_{T U}=\lambda_{T} \lambda_{U}$ for any $T, U \in L(X)$, and therefore that $\alpha$ is a group isomorphism of $H$ onto $\{-1,1\} \times G\left(\right.$ resp. $\left.\mathbb{C}_{1} \times G\right)$.

Next we recall that two Banach spaces are said to be totally incomparable if no infinite-dimensional subspace of one is isomorphic to a subspace of the other. It is clear that when two spaces are totally incomparable, any operator from one into another must be strictly singular.

Proposition 23. Let $S$ be a Banach space with a symmetric basis. Any group which is representable in $S \oplus X_{G M}$ is of the form $\{-1,1\} \times G$ in the real case (resp. $\mathbb{C}_{1} \times G$ in the complex case). In particular, in the real case, a countable group is representable in $S \oplus X_{G M}$ if and only if it is of the form $\{-1,1\} \times G$.

Proof. The last part of the proposition is a consequence of the initial part and of Theorem 21. We prove the initial part. Let $X=S \oplus X_{G M}$. We observe that $S$ and $X_{G M}$ are totally incomparable. Indeed every subspace of $S$ contains a subspace with an unconditional basis, while no subspace of $X_{G M}$ has an unconditional basis [10. Therefore any operator $T$ on $X$ may be written as a matrix of the form

$$
\left(\begin{array}{cc}
A & s_{1} \\
s_{2} & \lambda_{T} I d_{X_{G M}}+s
\end{array}\right)
$$

where $A \in L(S)$, and $s_{1} \in L\left(X_{G M}, S\right), s_{2} \in L\left(S, X_{G M}\right), s \in L\left(X_{G M}\right)$ are strictly singular, and $\lambda_{T} \neq 0$ if $T$ is an isomorphism. If $T$ is an isometry, then since $T_{\mid X_{G M}}$ is a strictly singular perturbation of $\lambda_{T} i_{X_{G M}, X}$, where $i_{X_{G M}, X}$ denotes the canonical injection of $X_{G M}$ into $X, \lambda_{T}$ must be of modulus 1, that is, belong to $\{-1,1\}$ (resp. $\left.\mathbb{C}_{1}\right)$. Furthermore we claim that for $T, U \in L(X), \lambda_{T U}=\lambda_{T} \lambda_{U}$. Indeed if $T$ is written as previously, and $U$ is written as

then $T U$ has the form

$$
\left(\begin{array}{cc}
B & s_{1}^{\prime} \\
s_{2}^{\prime} & \lambda_{U} I d_{X_{G M}}+s^{\prime}
\end{array}\right)
$$

$$
\left(\begin{array}{cc}
C & s_{1}^{\prime \prime} \\
s_{2}^{\prime \prime} & \lambda_{T} \lambda_{U} I d_{X_{G M}}+s_{2} s_{1}^{\prime}+\lambda_{T} s^{\prime}+\lambda_{U} s+s s^{\prime}
\end{array}\right),
$$

where $s_{2} s_{1}^{\prime}+\lambda_{T} s^{\prime}+\lambda_{U} s+s s^{\prime}$ is strictly singular.

Then let $H$ be the group of isometries on $S \oplus X_{G M}$ for some equivalent norm. Let $G$ be the subgroup of $H$ defined by $G=\left\{T \in H: \lambda_{T}=1\right\}$. Then mapping $T$ to $\left(\lambda_{T}, T / \lambda_{T}\right)$ we provide an isomorphism of $H$ onto the group $\{-1,1\} \times G$ (resp. $\left.\mathbb{C}_{1} \times G\right)$.

It remains open for a given separable infinite-dimensional real space $X$ exactly which finite (resp. countable) groups are representable. There are the maximal cases of $c_{0}, C([0,1]), \ell_{p}, 1 \leq p<+\infty$ or $L_{p}, 1 \leq p<+\infty$, in which all countable groups admitting a normal subgroup with two elements are representable, and the minimal case of $X_{G M}$, in which only groups of the form $\{-1,1\} \times G$ are representable. Apparently quite various situations may occur. Indeed we next show that a space constructed in 6 provides a third example which is "in between" the 
cases of $\ell_{p}$ and $X_{G M}$. This space, denoted $X(\mathbb{C})$, is, seen as real, the separable complex space defined in [6]. It has the property that every $\mathbb{R}$-linear operator on it is of the form $\lambda I d+S$, where $\lambda \in \mathbb{C}$ and $S$ is strictly singular as an $\mathbb{R}$-linear operator.

Proposition 24. The class of finite groups representable in $X(\mathbb{C})$ is neither equal to the class of finite groups which admit a normal subgroup with two elements, nor to the class of finite groups of the form $\{-1,1\} \times G$.

Proof. For any $n \in \mathbb{N}, n \geq 1$, the group $\left\{e^{i k \pi / 2 n} I d, 0 \leq k \leq 4 n-1\right\} \simeq \mathbb{Z} / 4 n \mathbb{Z}$ is a finite group of isomorphisms on $X(\mathbb{C})$ containing $-I d$. Therefore by Theorem 11 it is representable in $X(\mathbb{C})$; however it is not of the form $\{-1,1\} \times G$.

On the other hand, let $\{1, i, j, k\}$ be the generators of the algebra $\mathbb{H}$ of quaternions, and let $G$ be the group $\{ \pm 1, \pm i, \pm j, \pm k\}$. The group $\{-1,1\}$ is a normal subgroup of $G$ with two elements, and we prove that $G$ is not representable in $X(\mathbb{C})$.

Assume on the contrary that $\alpha$ is an isomorphism from $G$ onto $H$, where $H$ is the group of isometries on $X(\mathbb{C})$ in some equivalent norm. Since $-I d \in H,(-I d)^{2}=I d$ and -1 is the only element of square 1 in $G \backslash\{1\}$, we have $\alpha(-1)=-I d$. Therefore from $i j=-j i$ we deduce $\alpha(i) \alpha(j)=-\alpha(j) \alpha(i)$. Let, for $T$ an operator on $X(\mathbb{C})$, $\lambda_{T}$ be the unique complex number such that $T-\lambda_{T} I d$ is strictly singular. The map $T \mapsto \lambda_{T}$ induces a homomorphism of $H$ into $\mathbb{C}_{1}$. We deduce $\lambda_{\alpha(i)} \lambda_{\alpha(j)}=$ $-\lambda_{\alpha(j)} \lambda_{\alpha(i)}$, which is impossible in $\mathbb{C}_{1}$.

\section{Complex structures up to isometry}

We recall a few facts about complex structures, and we also refer to [5] for an introduction. A complex structure on a real Banach space $X$ is the space $X$ equipped with a $\mathbb{C}$-linear structure which induces the preexisting $\mathbb{R}$-linear structure on $X$. This may be seen in the isometric sense, that is, the complex structure is required to be $\mathbb{R}$-linearly isometric to $X$, or in the isomorphic sense, when the complex structure is required to be $\mathbb{R}$-linearly isomorphic to $X$.

A complex structure on $X$ is canonically associated to some $\mathbb{R}$-linear map $I$ on $X$ such that $I^{2}=-I d$, corresponding to the multiplication by $i$. Conversely for any such $I$, we may define the complex space $X^{I}$ with $\mathbb{C}$-linear structure defined by

$$
(a+i b) \cdot x=a x+b I x
$$

and equipped with the equivalent norm $\left\|\left|x\left\|\mid=\max _{\theta}\right\| \cos \theta x+\sin \theta I x \|\right.\right.$, so that $X^{I}$ is $\mathbb{R}$-linearly isomorphic to $X$. Therefore complex structures in the isomorphic sense correspond to operators of square $-I d$.

For a complex structure on $X$ in the isometric sense, we note that the map $I$ corresponding to the multiplication by $i$ is an isometry, as well as $\cos \theta I d+\sin \theta I$ for all $\theta$. Conversely for any such $I$, we may define the complex structure $X^{I}$ by $(a+i b) x=a x+b I x$, and note that $\left\|e^{i \theta} x\right\|=\|x\|$ for all $\theta$. Therefore the original norm on $X$ is a complex norm for $X^{I}$, and $X^{I}$ is $\mathbb{R}$-linearly isometric to $X$. So complex structures in the isometric sense correspond to isometries $I$ of square $-I d$ such that $\cos \theta I d+\sin \theta I$ is an isometry for all $\theta$ as well.

The following well-known lemma will be useful. 
Lemma 25. Let $X$ be a real Banach space, and let $X^{I}$ and $X^{J}$ be complex structures associated to operators $I$ and $J$, respectively. Then

(1) $X^{I}$ and $X^{J}$ are $\mathbb{C}$-linearly isomorphic if and only if there exists an $\mathbb{R}$-linear isomorphism $P$ on $X$ such that $I=P^{-1} J P$.

(2) $X^{I}$ and $X^{J}$ are $\mathbb{C}$-linearly isometric if and only if there exists an $\mathbb{R}$-linear isometry $P$ on $X$ such that $I=P^{-1} J P$.

Proof. $X^{I}$ and $X^{J}$ are $\mathbb{C}$-linearly isomorphic (resp. isometric) when there exists an $\mathbb{R}$-linear isomorphism (resp. isometry) $P$ on $X$ which is also $\mathbb{C}$-linear when seen as an operator from $X^{I}$ onto $X^{J}$. This last condition is equivalent to saying that

$$
P(I x)=J P x
$$

for all $x \in X$, or equivalently,

$$
I=P^{-1} J P
$$

A real space $X$ is said to have unique complex structure up to isomorphism if it admits complex structures in the isomorphic sense and all such complex structures on $X$ are isomorphic. This obviously does not depend on the choice of an equivalent norm on $X$.

On the other hand, $X$ is said to have unique complex structure up to isometry if it admits complex structures in the isometric sense and all such complex structures on $X$ are isometric. Of course this depends heavily on the norm on $X$.

In the introduction we mentioned several results about complex structures up to isomorphism, and we recall some of them here. First of all there are many examples of real spaces without complex structure up to isomorphism; the space of Gowers and Maurey is one of them. The space $\ell_{2}$ admits a unique complex structure up to isomorphism, and recently in [6], other examples with unique complex structure were provided, including an HI space and a space of the form $C(K)$. It was also proved by Bourgain in 1986 that there exist real spaces which admit several complex structures up to isomorphism; see 3 . More precisely recall that for a complex structure $X^{I}$ the conjugate $\overline{X^{I}}$ of $X^{I}$ is the space $X^{-I}$. That is, for a complex space $X$ with complex law $(\lambda, x) \mapsto \lambda$. $x$, the conjugate $\bar{X}$ of $X$ is $X$ equipped with the law $(\lambda, x) \mapsto \bar{\lambda}$. $x$. In particular $X$ is always $\mathbb{R}$-linearly isometric to $\bar{X}$. What Bourgain provided was an example of a complex space $X$ which is not $\mathbb{C}$-linearly isomorphic to its conjugate.

While it is not difficult to see that the space of Bourgain admits a continuum of nonisomorphic complex structures, an example of a space with exactly $n$ complex structures up to isomorphism, for any choice of $n \in \mathbb{N}, n \geq 2$, was provided in $[6$.

For complex structures up to isometry there are no explicit results in the literature. However we may observe that it is easy to renorm any space not to admit a complex structure in the isometric sense. To see this one may simply use the result of Jarosz [11] that any Banach space may be renormed to admit only trivial isometries. Therefore there are no isometries $I$ satisfying $I^{2}=-I d$ for that norm.

In this section we shall investigate possible renormings of real Banach spaces to admit complex structures up to isometry. This will be related to the problem of representation of groups in real Banach spaces, more precisely, to the representations of the circle group $\mathbb{C}_{1}$ and of the group $\operatorname{Isom}_{\mathbb{R}}(\mathbb{C})$ of $\mathbb{R}$-linear isometries on $\mathbb{C}$ as the group of isometries on a real Banach space. From this we shall obtain 
uniqueness or nonuniqueness properties of complex structures up to isometry on a Banach space, according to different choices of equivalent renormings.

We start with the study of the case of $\ell_{2}$ with its canonical norm.

3.1. The classical case of $\ell_{2}$. Recall that for a real Banach space $X$, the complexification $X \oplus_{\mathbb{C}} X$ of $X$ is the complex Banach space defined up to isomorphism as the sum $X \oplus X$ with the complex law

$$
(a+i b)(x, y)=(a x-b y, b x+a y),
$$

for $a, b \in \mathbb{R}$ and $x, y \in X$. Equivalently this is the complex structure $(X \oplus X)^{J}$ on the real space $X \oplus X$, where $J$ is defined by $J(x, y)=(-y, x)$. It is immediate to observe that the conjugate of $X \oplus_{\mathbb{C}} X$ is always $\mathbb{C}$-linearly isomorphic to $X \oplus_{\mathbb{C}} X$. From the isometric point of view, we shall see later on natural choices of the norm for which $X \oplus_{\mathbb{C}} X$ is actually $\mathbb{C}$-linearly isometric to its conjugate.

Here $\ell_{2}$ stands for the real separable Hilbert space. Associated to a given decomposition of this space as $\ell_{2} \oplus_{2} \ell_{2}$, there exists a canonical complex structure associated to the isometry $J$ defined on $\ell_{2}$ by $J(x, y)=(-y, x)$. In other words this complex structure is $\mathbb{C}$-linearly isometric to the complexification $\ell_{2} \oplus_{\mathbb{C}} \ell_{2}$ of $\ell_{2}$ equipped with the $\ell_{2}$-sum norm.

Proposition 26. The space $\ell_{2}$ admits a unique structure up to isometry.

Proof. It is enough to prove that whenever $A$ is an isometry on $\ell_{2}$ satisfying $A^{2}=$ $-I d, \ell_{2}^{A}$ is the canonical complex structure on $\ell_{2}$ associated to some decomposition of $\ell_{2}$ as $\ell_{2} \oplus_{2} \ell_{2}$, i.e. that there exists an orthonormal basis $\left(u_{n}\right)_{n \in \mathbb{N}}$ of $\ell_{2}$ such that decomposing $\ell_{2}=\left[\left(u_{2 n-1}\right)_{n \in \mathbb{N}}\right] \oplus\left[\left(u_{2 n}\right)_{n \in \mathbb{N}}\right]$ the matrix of $A$ is

$$
A=\left(\begin{array}{cc}
0 & -I d \\
I d & 0
\end{array}\right) \text {. }
$$

We note the following fact.

Fact. For any nonzero vector $x$ in $\ell_{2}$, there exists an orthonormal basis $\left\{u_{1}, u_{2}\right\}$ of $[x, A x]$ such that $A u_{1}=u_{2}$ and $A u_{2}=-u_{1}$. Furthermore the orthogonal subspace $\left[u_{1}, u_{2}\right]^{\perp}$ of $\left[u_{1}, u_{2}\right]$ is also invariant by $A$.

Proof. Since $A^{2}=-I d$, the subspace $[x, A x]$ generated by $x$ and $A x$ is invariant by $A$ and has dimension 2. Take an orthonormal basis $\{u, v\}$ of $[x, A x]$. Then the restriction of $A$ to $[u, v]$ is a rotation of angle $\theta$ for some $\theta \in \mathbb{R}$. Moreover, $\theta=\pi / 2$ or $\theta=3 \pi / 2$, because $A^{2}=-I d$. Therefore $A u=v$ and $A v=-u$ or $A u=-v$ and $A v=u$. Since the adjoint operator of $A$ is $-A$, the orthogonal subspace $\left[u_{1}, u_{2}\right]^{\perp}$ of $\left[u_{1}, u_{2}\right]$ is also invariant by $A$.

Now let $\left(e_{n}\right)_{n \in \mathbb{N}}$ be an orthonormal basis of $\ell_{2}$. In fact, there exists an orthonormal basis $\left\{u_{1}, u_{2}\right\}$ of $X_{1}=\left[e_{1}, A e_{1}\right]$ such that $A u_{1}=u_{2}$ and $A u_{2}=-u_{1}$. Pick $n_{1}$ to be the smallest $i \in \mathbb{N}$ satisfying $e_{i} \notin X_{1}$. Consequently $2 \leq n_{1}$.

We know that $\ell_{2}=X_{1} \oplus X_{1}^{\perp}$. So there exists some $f_{2} \in X_{1}^{\perp}$ satisfying $e_{n_{1}}-f_{2} \in$ $X_{1}$. Denote $X_{2}=\left[f_{2}, A f_{2}\right]$. By our observation, $X_{2} \subset X_{1}^{\perp}$. Now we can restrict $A$ to $X_{2}$, again by the fact there exists an orthogonal basis $\left\{u_{3}, u_{4}\right\}$ of $X_{2}$ such that $A u_{3}=u_{4}$ and $A u_{4}=-u_{3}$. Fix $n_{2}$ to be the smallest $i \in \mathbb{N}$ satisfying $e_{i} \notin X_{1} \oplus X_{2}$. Thus $3 \leq n_{2}$. 
Since $\ell_{2}=X_{1} \oplus X_{2} \oplus\left(X_{1} \oplus X_{2}\right)^{\perp}$, there exists $f_{3} \in\left(X_{1} \oplus X_{2}\right)^{\perp}$ satisfying $e_{n_{2}}-f_{3} \notin X_{1} \oplus X_{2}$.

Hence proceeding by induction, we can construct a sequence $\left(u_{n}\right)_{n \in \mathbb{N}}$ of normalized vectors in $\ell_{2}$ satisfying

- $A u_{2 n-1}=u_{2 n}$ and $A u_{2 n}=-u_{2 n-1}, \forall n \in \mathbb{N}$;

- $e_{n} \in \sum_{k=1}^{n}\left[u_{2 k-1}, u_{2 k}\right], \forall n \in \mathbb{N}$;

- $u_{i} \perp u_{j}, \forall i, j \in \mathbb{N}, i \neq j$.

Thus the proof of the proposition is complete.

Note that from this proposition we may deduce that $\ell_{2}$ also admits a unique complex structure up to isomorphism, a well-known fact for which there does not seem to be a written proof in the literature. Indeed if $A$ is an operator on $\ell_{2}$ satisfying $A^{2}=-I d$, let $\langle,\rangle^{\prime}$ be the scalar product defined on $\ell_{2}$ by $\langle x, y\rangle^{\prime}=$ $\langle x, y\rangle+\langle A x, A y\rangle$ and $\|.\|^{\prime}$ be the associated norm. Then $\left(\ell_{2},\|.\|^{\prime}\right)$ is a Hilbert space for which $A$ is an isometry, and therefore $\left(\ell_{2}^{A},\|\cdot\|^{\prime}\right)$ is isometric to the canonical complex structure on $\ell_{2}$ associated to a decomposition $\ell_{2}=\ell_{2} \oplus_{2} \ell_{2}$; hence $\left(\ell_{2}^{A},\|\cdot\|\right)$ is isomorphic to that complex structure.

In a first version of this paper, we mentioned as an open question whether the spaces $c_{0}$ and $\ell_{p}, p \neq 2$ admitted a unique complex structure up to isomorphism. N.J. Kalton then indicated to us a nice and simple proof that this is indeed the case. We reproduce this proof here with his authorization.

Recall that a Banach space $X$ is primary if $X \simeq Y$ or $X \simeq Z$ whenever $X=$ $Y \oplus Z$. The classical spaces $c_{0}, \ell_{p}, 1 \leq p \leq+\infty, L_{p}, 1<p<+\infty$, both real and complex, are known to be primary.

Lemma 27. Let $X$ be a real Banach space, and let $A, B$ be operators on $X$ such that $A^{2}=B^{2}=-I d$. Assume that $X^{A}$ is isomorphic to its conjugate and primary, and that $A$ and $B$ commute. Then $X^{A}$ and $X^{B}$ are isomorphic.

Proof. It is easily checked that $P=\frac{1}{2}(I d+A B)$ and $Q=\frac{1}{2}(I d-A B)$ are projections on $X$ which commute with $A$ and $B$, and such that $A x=-B x$ for any $x \in P X$, and $A x=B x$ for any $x \in Q X$. Let $X \simeq Y \oplus Z$ be the associated decomposition. Then $X^{A} \simeq Y^{A} \oplus Z^{A}$, and

$$
X^{B} \simeq Y^{B} \oplus Z^{B} \simeq Y^{-A} \oplus Z^{A} .
$$

Since $X^{A}$ is primary, we have either $Y^{A} \simeq X^{A}$ or $Z^{A} \simeq X^{A}$. In the first case, and since $X^{A}$ is isomorphic to its conjugate, we deduce

$$
X^{B} \simeq Y^{A} \oplus Z^{A} \simeq X^{A}
$$

In the second case,

$$
X^{B} \simeq Y^{-A} \oplus X^{A} \simeq Y^{-A} \oplus Z^{-A} \simeq X^{-A} \simeq X^{A}
$$

Theorem 28 (N.J. Kalton). Let $X$ be a real Banach space and assume that the complexification $X \oplus_{\mathbb{C}} X$ of $X$ is primary. Then $X$ admits no more than one complex structure up to isomorphism.

Proof. Let $J$ be the operator associated to the canonical complex structure on $X \oplus X$; i.e., $J$ is defined by $J(x, y)=(-y, x)$. Assume $X$ admits a complex structure and let $A$ be any operator on $X$ such that $A^{2}=-I d$. Let $A \oplus A$ denote 
the corresponding operator on $X \oplus X$. It is immediate that $A \oplus A$ and $J$ commute; therefore by Lemma 27 ,

$$
X^{A} \oplus X^{A} \simeq(X \oplus X)^{A \oplus A} \simeq(X \oplus X)^{J} .
$$

Since the space $(X \oplus X)^{J}$ is primary we deduce that

$$
X^{A} \simeq(X \oplus X)^{J},
$$

which concludes the proof.

Corollary 29. The real spaces $c_{0}(\mathbb{N}, \mathbb{R}), C([0,1], \mathbb{R}), \ell_{p}(\mathbb{N}, \mathbb{R}), 1 \leq p \leq+\infty$, $L_{p}([0,1], \mathbb{R}), 1 \leqslant p<+\infty$ admit a unique complex structure up to isomorphism.

Proof. We write the proof for the space $c_{0}(\mathbb{N}, \mathbb{R})$. The complexification of the real space $c_{0}(\mathbb{N}, \mathbb{R})$ is isomorphic to the complex space $c_{0}(\mathbb{N}, \mathbb{C})$, which is primary. Furthermore $c_{0}(\mathbb{N}, \mathbb{R})$, being isomorphic to its square, admits a complex structure, which is the canonical one associated to a decomposition $c_{0}(\mathbb{N}, \mathbb{R}) \simeq c_{0}(\mathbb{N}, \mathbb{R}) \oplus$ $c_{0}(\mathbb{N}, \mathbb{R})$. That is, the unique complex structure is the complexification of $c_{0}(\mathbb{N}, \mathbb{R})$, or equivalently, the complex space $c_{0}(\mathbb{N}, \mathbb{C})$, up to isomorphism. The same proof applies to the other examples.

3.2. Isometric complex structures up to renorming. For other spaces than $\ell_{2}$ with its usual norm, the theory of complex structures up to isometry turns out to be quite different from the theory up to isomorphism. For example, as we already mentioned, according to Jarosz in [11, any real Banach space admits an equivalent norm for which the only isometries are $I d$ and $-I d$. Therefore every real Banach space admits an equivalent norm for which it does not admit complex structures in the isometric sense. We shall now use the methods of Jarosz to prove:

Theorem 30. Any real Banach space of dimension at least 4 and which admits a complex structure up to isomorphism has an equivalent norm for which it admits exactly two complex structures up to isometry.

Any real Banach space which is isomorphic to a Cartesian square has an equivalent norm for which it admits a unique complex structure up to isometry.

Therefore $\ell_{2}$ with its usual norm is far from being the only Banach space with a unique complex structure up to isometry. Actually Theorem 30 and the result of Jarosz imply that all classical spaces may be renormed to have no, a unique, or exactly two complex structures up to isometry.

Note that the space $X(\mathbb{C})$ from [6, mentioned in Proposition 24, admits exactly two complex structures up to isomorphism, which are conjugate, and therefore cannot be renormed to admit a unique complex structure up to isometry. Indeed if $T^{2}=-I d$ for $T$ an isometry in some equivalent norm $\left\||\cdot \||\right.$ on $X(\mathbb{C})$, then $X(\mathbb{C})^{T}$ and $X(\mathbb{C})^{-T}$ are complex structures on $X(\mathbb{C})$ in the isomorphic sense, hence nonisomorphic and in particular non-|||.|||-isometric. This proves that the second part of Theorem 30 cannot be improved to all Banach spaces admitting a complex structure up to isomorphism.

It is quite clear that in any complex space, the maps $\lambda I d, \lambda \in \mathbb{C}_{1}$ are always $\mathbb{R}$-linear isometries. In many cases however, there is another class of "natural" real isometries. For example on $\mathbb{C}$, the conjugation map defined by $c(z)=\bar{z}$ and its multiples $\lambda c$ for $\lambda \in \mathbb{C}_{1}$ are real isometries; furthermore, any real isometry is either of the form $\lambda I d$ or $\lambda c, \lambda \in \mathbb{C}_{1}$. More generally, any complexification $Y \oplus_{\mathbb{C}} Y$ of a real space $Y$ admits renormings for which the conjugation map defined by 
$c(y, z)=(y,-z)$ is an isometric map, as well as $\lambda c$ for $\lambda \in \mathbb{C}_{1}$. This leads us to the following definitions.

Definition 31. Let $X$ be a complex space. We shall say that an $\mathbb{R}$-linear isometry $T$ on $X$ is trivial if $T=\lambda I d$, for some $\lambda$ in the complex unit circle.

Definition 32. We shall say that a complex space $X$ is regular for a complex norm $\|$.$\| on X$ if there exists an $\mathbb{R}$-linear subspace $Y$ of $X$ such that

(1) $X=Y \oplus i Y$, and

(2) the conjugation map $c$, defined by $c(y+i z)=y-i z$ for $(y, z) \in Y^{2}$, is an $\mathbb{R}$-linear isometry on $X$ for $\|$.$\| .$

Therefore a complex space $X$ is regular when $X$ is isomorphic to the complexification of some real subspace $Y$ and equipped with a norm for which the associated conjugation map is an isometry. A general example of regular complex spaces is the complexification of any real space $Y$, equipped with the equivalent norm

$$
\|y+i z\|=\sup _{\theta \in[0,2 \pi]}(\|y \cos \theta-z \sin \theta\|+\|z \cos \theta+y \sin \theta\|) .
$$

Other examples are the complex spaces $\ell_{p}(\mathbb{N}, \mathbb{C})$ or $L_{p}([0,1], \mathbb{C}), 1 \leq p<+\infty$, with their usual norms.

When we shall consider a regular complex space, it shall always be implicitly associated to a choice of decomposition $X=Y \oplus i Y$, and therefore $c$ will denote the conjugation map associated to that decomposition.

Definition 33. Let $X$ be a regular complex space. A real isometry $T$ on $X$ is said to be a conjugation isometry if it is of the form $T=\lambda c$, where $\lambda$ is in the unit complex circle and $c$ is the conjugation map.

The rest of this section is devoted to proving: (1) that any complex Banach space of dimension at least 2 may be renormed with a complex norm to admit only trivial real isometries (Corollary 45), and (2) that any complexification of a real Banach space may be renormed with a regular complex norm to admit only trivial and conjugation real isometries (Corollary 46). Theorem 30 follows immediately from Corollaries 45]and 46. Indeed in case (1) the only isometries of square $-I d$ are $i I d$ and $-i I d$. Furthermore since the group of isometries commutes, there is no $g$ in that group so that $g^{-1}(i I d) g=-i I d$, so the associated complex structures are not isometric; see Lemma 25. There are therefore exactly two complex structures up to isometry, which are conjugate. In case (2), since $T^{2}=|\lambda|^{2} I d$ whenever $T=\lambda c$, the isometries $i I d$ and $-i I d$ are also the unique isometries of square $-I d$. Since $-i I d=c(i I d) c=c^{-1}(i I d) c$, their associated complex structures are $\mathbb{C}$-linearly isometric. Therefore there is a unique complex structure up to isometry in that case.

Our proof of (1) and (2) consists in extending the methods of Jarosz concerning $\mathbb{C}$-linear isometries on complex spaces to the study of $\mathbb{R}$-linear isometries on complex spaces.

We first note that any equivalent complex norm on $\mathbb{C}$ is a multiple of the modulus; therefore real isometries on $\mathbb{C}$ are either trivial or conjugation isometries in any equivalent complex norm, and $\mathbb{C}$, as a $\mathbb{C}$-linear space, cannot be renormed to admit only trivial real isometries. We shall need a direct proof that the case of $\mathbb{C}^{2}$ is already different. 
Lemma 34. There exists a complex norm on $\mathbb{C}^{2}$ for which $\mathbb{C}^{2}$ only admits trivial real isometries.

Proof. We fix $\lambda_{0}=0$ and $\lambda_{k}, 1 \leq k \leq 4$, satisfying:

- i) $\left|\lambda_{k}\right|=1, \forall 1 \leq k \leq 4$,

- ii) $\operatorname{Re}\left(\lambda_{k}\right)>0, \forall 1 \leq k \leq 4$,

- iii) $\lambda_{j} \lambda_{k} \neq \lambda_{l} \lambda_{m}$ whenever $j, k, l, m \in\{1,2,3,4\}$ and $\{j, k\} \neq\{l, m\}$.

We define a norm $\|\cdot\|$ on $\mathbb{C}^{2}$ by the formula

$$
\|(x, y)\|=\max \left\{|x|, \max _{1 \leq k \leq 4}\left|x-\lambda_{k} y\right|\right\}=\max _{0 \leq k \leq 4}\left|x-\lambda_{k} y\right|,
$$

and we shall prove that any real isometry on $\mathbb{C}^{2}$ for that norm is trivial.

For $k=0,1,2,3,4$, let $A_{k}$ be the open subset of $\mathbb{C}^{2}$ defined by

$$
A_{k}=\left\{(x, y):\left|x-\lambda_{k} y\right|>\max _{0 \leq j \leq 4, j \neq k}\left|x-\lambda_{j} y\right|\right\},
$$

and let

$$
A=\bigcup_{0 \leq k \leq 4} A_{k} .
$$

Note that the sets $A_{k}$ are disjoint and that for $(x, y) \in A$, we have that $\|(x, y)\|=$ $\left|x-\lambda_{k} y\right|$ for a unique $k$. We let $H_{k}=\left\{\left(\lambda_{k} h, h\right), h \in \mathbb{C}\right\}$.

Claim. The set $A$ is the set of points $(x, y)$ such that $\|$.$\| is constant in a neighbor-$ hood of $(x, y)$ in $(x, y)+H$ for some (unique) $\mathbb{R}$-linear subspace $H$ of $\mathbb{R}$-dimension 2. So $A$ is defined by $\mathbb{R}$-linear and metric properties.

Proof. We observe that if $(x, y) \in A_{k}$, then $\|x, y\|$ is equal to $\left|x-\lambda_{k} y\right|$ and is constant in a neighborhood $(x, y)+V_{k}$ of $(x, y)$ in $(x, y)+H$, for some linear subspace $H$ of $\mathbb{R}$-dimension 2: $H=H_{k}$ will do. Note that $H=H_{k}$ is the only $H$ sharing this property.

On the other hand, when $(x, y) \notin A$, let $j \neq k$ be such that $\|(x, y)\|=\left|x-\lambda_{j} y\right|=$ $\left|x-\lambda_{k} y\right|$. Assume that $\|$.$\| is constant on a neighborhood (x, y)+V$ of $(x, y)$ in $(x, y)+H$, for some linear subspace $H$ of $\mathbb{R}$-dimension 2. Let $g$ be defined for $\left(h, h^{\prime}\right) \in V$ by $g\left(h, h^{\prime}\right)=\left|x+h-\lambda_{k}\left(y+h^{\prime}\right)\right|$. Then

$$
g\left(h, h^{\prime}\right) \leq\left\|x+h, y+h^{\prime}\right\|=\|(x, y)\|
$$

and $g(0,0)=\|(x, y)\|$. So $g\left(h, h^{\prime}\right)$ attains its maximum $\|(x, y)\|=\left|x-\lambda_{k} y\right|$ on $V$ in $(0,0)$. Without loss of generality we may assume that $V=-V$ and we observe that for $\left(h, h^{\prime}\right) \in V$,

$$
g\left(h, h^{\prime}\right)=\left|x-\lambda_{k} y+\left(h-\lambda_{k} h^{\prime}\right)\right| \leq|x-\lambda y|,
$$

and

$$
g\left(-h,-h^{\prime}\right)=\left|x-\lambda_{k} y-\left(h-\lambda_{k} h^{\prime}\right)\right| \leq|x-\lambda y|,
$$

from which we deduce easily that $h-\lambda_{k} h^{\prime}=0$, that is, $\left(h, h^{\prime}\right) \in H_{k}$. So $V \subset H_{k}$ and therefore $H=H_{k}$. But then by the same reasoning, $H=H_{j}$, so $\lambda_{j}=\lambda_{k}$, a contradiction since $j \neq k$.

Finally we have proved that a point $(x, y)$ belongs to $A$ if and only if $\|\cdot\|$ is constant in a neighborhood of $(x, y)$ in $(x, y)+H$, for some $\mathbb{R}$-linear subspace $H$ of $\mathbb{R}$-dimension 2 . 
Let $T$ be an $\mathbb{R}$-linear isometry on $\left(\mathbb{C}^{2},\|\|.\right)$. By the claim, $T$ preserves $A$. Let $(x, y) \in A_{k}, 0 \leq k \leq 4$ and let $l$ be such that $T(x, y) \in A_{l}$. Since

$$
\|T(x, y)\|=\|(x, y)\|=\left\|(x, y)+\left(h, h^{\prime}\right)\right\|=\left\|T(x, y)+T\left(h, h^{\prime}\right)\right\|,
$$

for $\left(h, h^{\prime}\right) \in V_{k}$, it follows that $T V_{k}$ is a neighborhood of $T(x, y)$ in $T(x, y)+$ $\operatorname{span}_{\mathbb{R}} T V_{k}$ on which $\|$.$\| is constant. Since T(x, y) \in A_{l}$ and by the uniqueness of $H$ in the claim,

$$
T H_{k}=\operatorname{span}_{\mathbb{R}} T V_{k}=H_{l} .
$$

Since $(x, y)$ was arbitrary in $A_{k}$, this means that there exists a unique $l$ such that $T(x, y) \in A_{l}$ for $(x, y) \in A_{k}$, and therefore that $T\left(A_{k}\right) \subset A_{l}$ for that $l$. So $T\left(A_{k}\right)=A_{l}$ by considering the isometry $T^{-1}$, and finally there is a permutation $\sigma$ on $\{0,1,2,3,4\}$ such that $T\left(A_{k}\right)=A_{\sigma(k)}$ for all $0 \leq k \leq 4$.

The isometry $T$ is given by a formula of the form

$$
T(x, y)=(A x+B \bar{x}+C y+D \bar{y}, a x+b \bar{x}+c y+d \bar{y}),
$$

where $A, B, C, D, a, b, c, d$ are complex numbers.

For any $k=1,2,3,4$ and any $\theta \in[0,2 \pi]$, we have that $\left(e^{i \theta},-\frac{t}{\lambda_{k}} e^{i \theta}\right)$ belongs to $A_{k}$ for $t>0$, so $e^{i \theta} \in \overline{A_{k}}$. Likewise by condition ii) it is easy to check that $\left(e^{i \theta},-t e^{i \theta}\right)$ belongs to $A_{0}$ for $t>0$, so $e^{i \theta} \in \overline{A_{0}}$.

Finally $\left(e^{i \theta}, 0\right) \in \bigcap_{0 \leq k \leq 4} \overline{A_{k}}$. By our computation of $T\left(A_{k}\right), 0 \leq k \leq 4$, we have therefore

$$
T\left(e^{i \theta}, 0\right)=\left(A e^{i \theta}+B e^{-i \theta}, a e^{i \theta}+b e^{-i \theta}\right) \in \bigcap_{0 \leq k \leq 4} \overline{A_{k}},
$$

and we deduce that for any $\theta$ and any $k=0,1,2,3,4$,

$$
1=\left\|T\left(e^{i \theta}, 0\right)\right\|=\left|\left(A-\lambda_{k} a\right) e^{i \theta}+\left(B-\lambda_{k} b\right) e^{-i \theta}\right|=\left|\left(A-\lambda_{k} a\right) e^{2 i \theta}+\left(B-\lambda_{k} b\right)\right| .
$$

We deduce easily that either $A-\lambda_{k} a=0$ for at least two values of $k$, so that $A=a=0$ and $\left|B-\lambda_{k} b\right|=|B|=1$ for all $1 \leq k \leq 4$, so also $b=0$, or that $B-\lambda_{k} b=0$ for at least two values of $k$, so that $B=b=0$ and similarly $|A|=1$, $a=0$.

Likewise for any $\theta,\left(0, e^{i \theta}\right) \in \bigcap_{1 \leq k \leq 4} \overline{A_{k}}$. Then $T\left(0, e^{i \theta}\right)=\left(C e^{i \theta}+D e^{-i \theta}, c e^{i \theta}+\right.$ $\left.d e^{-i \theta}\right) \in \bigcap_{0 \leq k \leq 4, k \neq \sigma(0)} \overline{A_{k}}$, so we deduce

$$
1=\left|\left(C-\lambda_{k} c\right) e^{i \theta}+\left(D-\lambda_{k} d\right) e^{-i \theta}\right|, \forall 0 \leq \theta \leq 2 \pi, \forall k=0,1,2,3,4, k \neq \sigma(0) .
$$

So either $C=c=0$ in which case $\left|D-\lambda_{k} d\right|=1$ for all $k=1,2,3,4, k \neq \sigma(0)$, from which it follows easily that $d=0$ or $D=0$, or $D=d=0$ and $(C=0$ or $c=0)$.

Summing up we have obtained that $T$ is given either by (1) $T(x, y)=(A x, c y)$, (2) $T(x, y)=(A x, d \bar{y}),(3) T(x, y)=(B \bar{x}, c y)$, or $(4) T(x, y)=(B \bar{x}, d \bar{y})$. It remains to prove that only (1) is possible, with $A=c$. Without loss of generality we may assume $A=B=1$, and we have $|c|=|d|=1$.

For any $0 \leq \theta \leq 2 \pi$, we observe that

$$
2=\left\|\left(e^{i \theta},-\overline{\lambda_{1}} e^{i \theta}\right)\right\| .
$$

If $T$ satisfies (2), then we deduce

$$
2=\left\|\left(e^{i \theta},-d \lambda_{1} e^{-i \theta}\right)\right\|=\max \left(1, \max _{1 \leq k \leq 4}\left|e^{i \theta}+\lambda_{k} \lambda_{1} d e^{-i \theta}\right|\right) .
$$

So $2=\max _{1 \leq k \leq 4}\left|e^{i 2 \theta}+\lambda_{k} \lambda_{1} d\right|$, but obviously this is only possible for a finite number of values of $\theta$, so we get a contradiction. A similar reasoning holds to exclude the case (3). 
If $T$ satisfies (4), then for any $j=1,2,3,4$, and any $\theta$,

$$
2=\left\|\left(e^{-i \theta},-d \lambda_{j} e^{-i \theta}\right)\right\|=\max \left(1, \max _{1 \leq k \leq 4}\left|e^{-i \theta}+\lambda_{k} \lambda_{j} d e^{-i \theta}\right|\right) .
$$

So $2=\max _{1 \leq k \leq 4}\left|1+\lambda_{k} \lambda_{j} d\right|$. We deduce that for any $j=1,2,3,4$, there exists $k \geq 1$ such that $\lambda_{j} \lambda_{k}=1 / d$, but this contradicts condition iii) on the $\lambda_{k}$ 's.

So $T$ satisfies (1) and assuming $A=1$ it remains to prove that $c=1$. We have that

$$
2=\left\|\left(e^{i \theta},-c \overline{\lambda_{j}} e^{i \theta}\right)\right\|=\max \left(1, \max _{1 \leq k \leq 4}\left|e^{i \theta}+\lambda_{k} \overline{\lambda_{j}} c e^{i \theta}\right|\right) .
$$

So $2=\max _{1 \leq k \leq 4}\left|1+\lambda_{k} \overline{\lambda_{j}} c\right|$. We deduce that for any $j=1,2,3,4$, there exists $k \geq 1$ such that $\lambda_{j}=c \lambda_{k}$. But then $c=1$; otherwise there exist $k=2,3$ or 4 and $k^{\prime}=1,2,3$ or 4 such that $\lambda_{1}=c \lambda_{k}$ and $\lambda_{k}=\lambda_{k^{\prime}}$. This would imply that $\lambda_{1} / \lambda_{k}=\lambda_{k} / \lambda_{k^{\prime}}$, contradicting condition iii).

Note that from the definition of the norm it is clear that for $x \in \mathbb{C},\|x, 0\|=|x|$. This fact will be used at the end of this article.

In the following we shall consider certain complex spaces $E$ satisfying

$$
c_{0}(\Gamma, \mathbb{C}) \subset E \subset \ell_{\infty}(\Gamma, \mathbb{C}),
$$

for some nonempty set $\Gamma$. Such a space $E$ is therefore equipped with the induced sup norm $\|$.$\| . For \gamma \in \Gamma$ we let $e_{\gamma} \in E$ be the characteristic function of $\{\gamma\}$. We start with two lemmas characterizing $\mathbb{R}$-linear isometries on such an $E$.

Lemma 35. Let $\Gamma$ be a nonempty set and $E$ a complex Banach space with norm $\|$.$\| such that c_{0}(\Gamma, \mathbb{C}) \subset E \subset \ell_{\infty}(\Gamma, \mathbb{C})$. Let $T$ be an $\mathbb{R}$-linear isometry on $E$. Then there exists a bijection $\pi$ on $\Gamma$ and coefficients $\epsilon_{\gamma}$, with $\left|\epsilon_{\gamma}\right|=1$, such that for each $\gamma \in \Gamma$, either

(1) $T\left(\lambda e_{\gamma}\right)=\epsilon_{\gamma} \lambda e_{\pi(\gamma)}$, for any $\lambda \in \mathbb{C}$, or

(2) $T\left(\lambda e_{\gamma}\right)=\epsilon_{\gamma} \bar{\lambda} e_{\pi(\gamma)}$, for any $\lambda \in \mathbb{C}$.

Proof. We adapt a proof suggested by the referee. We claim the following.

Claim. Two normalized vectors $x, y$ in $E$ have disjoint supports if and only if

$$
\begin{gathered}
\forall z \in E,\|x+y+z\|=\max \{\|x+z\|,\|y+z\|\}, \\
\text { and }\|x-y+z\|=\max \{\|x+z\|,\|y-z\|\} .
\end{gathered}
$$

Proof. The "only if" part is obvious. To prove the "if" part, suppose that $\gamma \in$ $\operatorname{supp}(x) \cap \operatorname{supp}(y)$. Up to exchanging the roles of $x$ and $y$ assume that $\left|y_{\gamma}\right| \leq\left|x_{\gamma}\right|$. By the triangle inequality we cannot have $\left|x_{\gamma}\right|>\left|x_{\gamma}+y_{\gamma}\right|$ and $\left|x_{\gamma}\right|>\left|x_{\gamma}-y_{\gamma}\right|$, so up to replacing $y$ by $-y$ we may assume that $\left|x_{\gamma}\right| \leq\left|x_{\gamma}+y_{\gamma}\right|$. Finally up to replacing $x$ and $y$ by $\lambda x$ and $\lambda y$ for some $\lambda \in \mathbb{C}_{1}$ we may also assume that $x_{\gamma}+y_{\gamma} \in \mathbb{R}$.

We let $z=2 e_{\gamma} \in c_{0}(\Gamma, \mathbb{C}) \subset E$. We see that

$$
\|x+y+z\| \geq\left|x_{\gamma}+y_{\gamma}+2\right|=x_{\gamma}+y_{\gamma}+2 \geq\left|x_{\gamma}\right|+2 .
$$

First this implies that $\|x+y+z\| \geq\left|x_{\gamma}+2\right|$. We claim that this inequality is strict. Otherwise the chain of inequalities becomes

$$
x_{\gamma}+y_{\gamma}+2=\left|x_{\gamma}\right|+2=\left|x_{\gamma}+2\right|,
$$

which implies that $x_{\gamma} \in \mathbb{R}^{+}$and therefore that $y_{\gamma}=0$, which contradicts $\gamma \in$ $\operatorname{supp}(y)$. So the claim that $\|x+y+z\|>\left|x_{\gamma}+2\right|$ is proved. 
We also deduce that

$$
\|x+y+z\| \geq\left|x_{\gamma}\right|+2>\|x\| \geq\left|x_{\mu}\right|,
$$

for every $\mu \in \Gamma, \mu \neq \gamma$, since $x$ is a normalized vector. Consequently

$$
\|x+y+z\|>\max \left(\left|x_{\gamma}+2\right|, \max _{\mu \neq \gamma}\left|x_{\mu}\right|\right)=\|x+z\| .
$$

Likewise

$$
\|x+y+z\|>\|y+z\| .
$$

This therefore proves the "if part", and concludes the proof of the claim.

Since two vectors $x$ and $y$ have disjoint supports if and only if $x /\|x\|$ and $y /\|y\|$ satisfy the claim, we see therefore that "having disjoint supports" is preserved by $\mathbb{R}$-linear isometries.

Now fix $T$ to be an $\mathbb{R}$-linear isometry for $\|$.$\| on E$. Therefore $T$ maps disjointly supported vectors to disjointly supported vectors. It follows that for any $\gamma \in \Gamma$, $T$ cannot map $e_{\gamma}$ to a vector of the form $x+y, x, y$ nonzero vectors of disjoint supports. Otherwise $e_{\gamma}=T^{-1} x+T^{-1} y$ would be decomposed as the sum of two nonzero vectors with disjoint supports, by the claim applied to $T^{-1}$, which is impossible. It follows that $T$ maps $e_{\gamma}$ to some $\epsilon_{\gamma} e_{\pi(\gamma)}$, where $\pi: \Gamma \rightarrow \Gamma$ and $\left|\epsilon_{\gamma}\right|=1$.

By the same reasoning, $T^{-1}$ maps each $e_{\gamma}$ to some $\nu_{\gamma} e_{\rho(\gamma)}$. Therefore

$$
e_{\gamma}=T T^{-1} e_{\gamma}=\nu_{\gamma} \epsilon_{\rho(\gamma)} e_{\pi \rho(\gamma)},
$$

so $\pi \rho=I d_{\Gamma}$. Likewise $\rho \pi=I d_{\Gamma}$ and therefore, $\pi$ is a bijection.

Finally since $T i$ is also an $\mathbb{R}$-linear isometry, it also follows that $T$ maps $i e_{\gamma}$ to some $\epsilon_{\gamma}^{\prime} i e_{\pi^{\prime}(\gamma)}$, where $\pi^{\prime}: \Gamma \rightarrow \Gamma$ and $\left|\epsilon_{\gamma}^{\prime}\right|=1$. Since for all $\theta \in \mathbb{R}$,

$$
1=\left\|T\left(e^{i \theta} e_{\gamma}\right)\right\|=\left\|\cos \theta \epsilon_{\gamma} e_{\pi(\gamma)}+i \sin \theta \epsilon_{\gamma}^{\prime} e_{\pi^{\prime}(\gamma)}\right\|,
$$

it follows that $\pi^{\prime}(\gamma)=\pi(\gamma)$; otherwise $1=\max (\cos \theta, \sin \theta)$ for all $\theta$. Since

$$
1=\left|\cos \theta \epsilon_{\gamma}+i \sin \theta \epsilon_{\gamma}^{\prime}\right| \text {, }
$$

for all $\theta$, it also follows that $\epsilon_{\gamma}^{\prime}= \pm \epsilon_{\gamma}$. When $\epsilon_{\gamma}^{\prime}=\epsilon_{\gamma}$, we have that

$$
T\left(\lambda e_{\gamma}\right)=\epsilon_{\gamma} \lambda e_{\pi(\gamma)},
$$

for any $\lambda \in \mathbb{C}$, and when $\epsilon_{\gamma}^{\prime}=-\epsilon_{\gamma}$, that

$$
T\left(\lambda e_{\gamma}\right)=\epsilon_{\gamma} \bar{\lambda} e_{\pi(\gamma)},
$$

for any $\lambda \in \mathbb{C}$. This concludes the proof of the lemma.

Lemma 36. Let $\Gamma$ be a nonempty set and $E$ a complex Banach space with norm $\|$. such that $c_{0}(\Gamma, \mathbb{C}) \subset E \subset \ell_{\infty}(\Gamma, \mathbb{C})$. Let $T$ be an $\mathbb{R}$-linear isometry on $E$. Assume that there exists $\lambda \in \mathbb{C}$ such that $T\left(e_{\gamma}\right)=\lambda e_{\gamma}$ and $T\left(i e_{\gamma}\right)=i \lambda e_{\gamma}$ for all $\gamma \in \Gamma$. Then $T=\lambda I d$.

Proof. For any $\left(a_{\gamma}\right)_{\gamma} \in E$, write $\left(b_{\gamma}\right)_{\gamma}=T\left(\left(a_{\gamma}\right)_{\gamma}\right)$. Then we have for fixed $\gamma \in \Gamma$ and any $r \in \mathbb{C}$,

$$
\left\|\left(a_{\gamma}\right)_{\gamma}-r e_{\gamma}\right\|=\left\|\left(b_{\gamma}\right)_{\gamma}-\lambda r e_{\gamma}\right\| .
$$

Since this norm is the sup norm, when $|r|$ is large enough this means that $\left|b_{\gamma}-\lambda r\right|=$ $\left|a_{\gamma}-r\right|$ and therefore $b_{\gamma}=\lambda a_{\gamma}$, and this holds for any $\gamma \in \Gamma$. Therefore $T=$ $\lambda I d$. 
We now consider a version of Lemma 36 which characterizes conjugation isometries instead of trivial isometries. To do this, we observe that associated to the inclusion $\mathbb{R} \subset \mathbb{C}$ there is a natural inclusion $\ell_{\infty}(\Gamma, \mathbb{R}) \subset \ell_{\infty}(\Gamma, \mathbb{C})$ and a decomposition of $\ell_{\infty}(\Gamma, \mathbb{C})$ as $\ell_{\infty}(\Gamma, \mathbb{R}) \oplus i \ell_{\infty}(\Gamma, \mathbb{R})$. In other words, $\ell_{\infty}(\Gamma, \mathbb{C})$ is $\mathbb{C}$-linearly isometric to the complexification of $\ell_{\infty}(\Gamma, \mathbb{R})$ with the associated complex law and the norm defined by

$$
\left\|\left(x_{\gamma}\right)_{\gamma}+i\left(y_{\gamma}\right)_{\gamma}\right\|=\sup _{\gamma \in \Gamma}|x(\gamma)+i y(\gamma)| .
$$

In particular the conjugation map $c$, defined for $x, y$ in $\ell_{\infty}(\Gamma, \mathbb{R})$ by $c(x+i y)=x-i y$ is a real isometry, and so the space $\ell_{\infty}(\Gamma, \mathbb{C})$ is regular with respect to the above decomposition. Therefore we may talk about a conjugation isometric map $c$, which is here defined by

$$
c\left(\left(a_{\gamma}\right)_{\gamma \in \Gamma}\right)=\left(\overline{a_{\gamma}}\right)_{\gamma \in \Gamma},
$$

and about conjugation isometries on $\ell_{\infty}(\Gamma, \mathbb{C})$.

In the same way, if $X$ is a subspace of $\ell_{\infty}(\Gamma, \mathbb{R})$, then the subspace $X \oplus i X$ of $\ell_{\infty}(\Gamma, \mathbb{C})$ is regular with respect to the decomposition $X \oplus i X$, and therefore we may also talk about conjugation isometries on $X \oplus i X$. If furthermore $c_{0}(\Gamma, \mathbb{R}) \subset X$, then we shall have that $c_{0}(\Gamma, \mathbb{C}) \subset X \oplus i X$.

Lemma 37. Let $\Gamma$ be a nonempty set and $X$ a real Banach space with norm $\|$. such that $c_{0}(\Gamma, \mathbb{R}) \subset X \subset \ell_{\infty}(\Gamma, \mathbb{R})$. Let $E=X \oplus i X \subset \ell_{\infty}(\Gamma, \mathbb{C})$, and let $T$ be an $\mathbb{R}$-linear isometry on $E$. Assume that there exists $\lambda \in \mathbb{C}$ such that $T\left(e_{\gamma}\right)=\lambda e_{\gamma}$ and $T\left(i e_{\gamma}\right)=-i \lambda e_{\gamma}$ for all $\gamma \in \Gamma$. Then $T=\lambda c$.

Proof. For any $\left(a_{\gamma}\right)_{\gamma} \in E$, write $\left(b_{\gamma}\right)_{\gamma}=T\left(\left(a_{\gamma}\right)_{\gamma}\right)$. Then we have for fixed $\gamma \in \Gamma$ and any $r \in \mathbb{C}$,

$$
\left\|\left(a_{\gamma}\right)_{\gamma}-r e_{\gamma}\right\|=\left\|\left(b_{\gamma}\right)_{\gamma}-\lambda \bar{r} e_{\gamma}\right\| .
$$

Since this norm is the sup norm, when $|r|$ is large enough this means $\left|\overline{b_{\gamma}}-\bar{\lambda} r\right|=$ $\left|b_{\gamma}-\lambda \bar{r}\right|=\left|a_{\gamma}-r\right|$ and therefore $b_{\gamma}=\lambda \overline{a_{\gamma}}$, and this holds for any $\gamma \in \Gamma$. Therefore $T=\lambda c$.

We now pass to the following crucial proposition, which imitates the result of Jarosz about complex trivial isometries (see [11, Proposition 1) in the following manner. Using the norm defined there, and in the case of a regular complex space, we obtain a result concerning trivial and conjugation real isometries, case (2). In other words the construction of Jarosz preserves real conjugation isometries, when they exist. To obtain a result concerning only trivial real isometries, one needs to add an ingredient in the definition of the norm, case (1). Intuitively this means that some symmetry must be broken if one wishes to "kill" conjugation isometries.

Proposition 38. Let $\Gamma$ be a nonempty set and let $E$ be a complex Banach space with norm $\|$.$\| such that c_{0}(\Gamma, \mathbb{C}) \subset E \subset \ell_{\infty}(\Gamma, \mathbb{C})$. Then

(1) if $|\Gamma| \geq 2$, then there is a complex norm $\left\||\cdot \||_{1}\right.$ on $E$, equivalent with the original sup norm $\|$.$\| of E$ and such that an $\mathbb{R}$-linear map $T$ on $E$ is both $a\|$.$\| and \|\|^{.\|\|_{1}}$ isometry if and only if $T$ is trivial;

(2) if $E=X \oplus i X$, where $X$ is a some real Banach space such that $c_{0}(\Gamma, \mathbb{R}) \subset$ $X \subset \ell_{\infty}(\Gamma, \mathbb{R})$, then there is a complex norm \|\|$_{\|} \|_{2}$ on $E$, equivalent with the original norm $\|$.$\| of E$ and such that an $\mathbb{R}$-linear map $T$ on $E$ is both a $\|$.$\| and \|\mid \cdot\|_{2}$ isometry if and only if $T$ is a trivial or conjugation isometry. 
Proof. We start by proving the easier case (2). If $|\Gamma|=1$, then $E=\mathbb{R} \oplus \mathbb{R}$ with the norm $\|x, y\|=|x+i y|$, or in other words $E=\mathbb{C}$. Since the $\mathbb{R}$-linear $\|\cdot\|$-isometries on $\mathbb{C}$ are the trivial and the conjugation isometries, it is clear that (2) holds with $\left\|\left|\cdot\left\|\left.\right|_{2}=\right\| . \|\right.\right.$. Therefore we may assume that $| \Gamma \mid \geq 2$. We then fix a well-order $<$ on $\Gamma$ and define

$$
\|x\|_{2}=\max (\|x\|, \sup |2 x(\gamma)+x(\beta)|, \gamma<\beta \in \Gamma) .
$$

Assume $T$ is an $\mathbb{R}$-linear isometry for $\|\cdot\|$ and $\|\cdot\|_{2}$. By Lemma 35 we know that $T e_{\gamma}=\epsilon_{\gamma} e_{\pi(\gamma)}$ for all $\gamma \in \Gamma$, for some bijective map $\pi$ on $\Gamma$ and some coefficients $\epsilon_{\gamma}$ of modulus 1 .

If $\gamma<\gamma^{\prime}$ but $\pi(\gamma)>\pi\left(\gamma^{\prime}\right)$, then $\left\|2 e_{\gamma}+e_{\gamma^{\prime}}\right\|_{2}=5$ but $\left\|T\left(2 e_{\gamma}+e_{\gamma^{\prime}}\right)\right\|_{2}=$ $\left\|\epsilon_{\gamma^{\prime}} e_{\pi\left(\gamma^{\prime}\right)}+2 \epsilon_{\gamma} e_{\pi(\gamma)}\right\|_{2} \leq 4$, a contradiction. So $\pi$ preserves order and is therefore equal to $I d_{\Gamma}$. If $\epsilon_{\gamma} \neq \epsilon_{\gamma^{\prime}}$ for $\gamma<\gamma^{\prime}$, then $\left\|e_{\gamma}+e_{\gamma^{\prime}}\right\|_{2}=3$, but

$$
\left\|T\left(e_{\gamma}+e_{\gamma^{\prime}}\right)\right\|_{2}=\left\|\epsilon_{\gamma} e_{\gamma}+\epsilon_{\gamma^{\prime}} e_{\gamma^{\prime}}\right\|_{2} \leq \max \left\{1,2,\left|2 \epsilon_{\gamma}+\epsilon_{\gamma^{\prime}}\right|\right\}<3 .
$$

Hence $\epsilon_{\gamma}$ is constant on $\Gamma$.

We have finally obtained that for some $\lambda= \pm \mu,|\lambda|=1$, and for all $\gamma \in \Gamma$, $T\left(e_{\gamma}\right)=\lambda e_{\gamma}$ and $T\left(i e_{\gamma}\right)=\mu i e_{\gamma}$. If $\lambda=\mu$, then we deduce from Lemma 36 that $T$ is the trivial isometry $\lambda I d$, and if $\lambda=-\mu$, then from Lemma 37 we deduce that $T$ is the conjugation isometry $\lambda c$.

To prove (1), as $|\Gamma| \geq 2$ we may fix some $\gamma_{0}<\gamma_{1}$ and consider the norm defined by

$$
\|x\|_{1}=\max \left(\left\||x \||_{2},\left|\frac{3}{2} x\left(\gamma_{0}\right)+i x\left(\gamma_{1}\right)\right|\right) .\right.
$$

Let $T$ be an $\mathbb{R}$-linear isometry for $\|$.$\| and \|.\|_{1}$. Since the new term $\left|\frac{3}{2} x\left(\gamma_{0}\right)+i x\left(\gamma_{1}\right)\right|$ in this expression has modulus at most $5 / 2$, which is smaller than the estimates $3,4,5$ used previously, it is easy to check that the reasoning used for $\|\cdot\|_{2}$ applies here to obtain that for some $\lambda= \pm \mu,|\lambda|=1$, and for all $\gamma \in \Gamma, T\left(e_{\gamma}\right)=\lambda e_{\gamma}$ and $T\left(i e_{\gamma}\right)=\mu i e_{\gamma}$. Furthermore, since

$$
\left\|e_{\gamma_{0}}+i e_{\gamma_{1}}\right\|_{1}=\max \left(1,2, \sqrt{5}, \frac{1}{2}\right)=\sqrt{5}
$$

but

$$
\left\|e_{\gamma_{0}}-i e_{\gamma_{1}}\right\|_{1}=\max \left(1,2, \sqrt{5}, \frac{5}{2}\right)=\frac{5}{2} \neq \sqrt{5}
$$

so $T\left(i e_{\gamma_{1}}\right)$ may not be equal to $-\lambda i e_{\gamma_{1}}$. This means that $\lambda=\mu$ and therefore that $T$ is equal to the trivial isometry $\lambda I d$.

Observe that if $|\Gamma|=1$, then $E=\mathbb{C}$. It is clearly not possible to renorm $\mathbb{C}$ with a complex norm to admit only trivial real isometries. Indeed any equivalent complex norm on $\mathbb{C}$ is a multiple of the modulus and therefore must admit conjugation isometries. So the condition that $|\Gamma| \geq 2$ in Proposition 38 (1) was necessary.

The next lemma and propositions are a version of Proposition 3 from [11: the results from [11] about $\mathbb{C}$-linear isometries on complex spaces are extended to $\mathbb{R}$ linear isometries on complex spaces. A great part of their proof is identical to the proof of [11, Proposition 3. In particular, the definition of the norm $\|\cdot\|_{W}$ is the same, as well as some arguments, although these are developed in much more detail in our paper. 
Lemma 39. Let $(X,\|\|$.$) be a complex Banach space, x_{0}$ a nonzero element of $X$, and $p($.$) a continuous complex norm on (X,\|\cdot\|)$. Then there exists a complex norm $\|\cdot\|_{w}$ on $Y=X \oplus \mathbb{C}$ such that $\|\cdot\|_{w}$ and $\|\cdot\|$ coincide on $X$ and such that for any $\mathbb{R}$-linear isometry $T$ on $Y$ for $\|\cdot\|_{w}$,

(1) $T X=X$,

(2) $T_{\mid X}$ is an isometry for $\|$.$\| ,$

(3) $T_{\mid X}$ is an isometry for $p($.$) ,$

(4) there exists $\lambda \in \mathbb{C}$ such that $T\left(x_{0}, 0\right)=\lambda\left(x_{0}, 0\right)$ and $T(0,1)=(0, \lambda)$.

Proof. By replacing $p($.$) by p()+.\|$.$\| and multiplying by an appropriate number, we$ may assume that $p$ and $\|$.$\| are equivalent, that 1000\|.\| \leq p($.$) and that \left\|x_{0}\right\| \leq 0.1$. Let

$$
\begin{aligned}
& A=\{(x, t) \in X \oplus \mathbb{C}=Y: \max \{\|x\|,|t|\} \leq 1\}, \\
& C=\left\{\left(x+x_{0}, 2\right): p(x) \leq 1\right\},
\end{aligned}
$$

and let $\|\cdot\|_{W}$ be the norm whose unit ball $W$ is the closed balanced convex set generated by $A \cup C$, that is,

$$
W=\overline{\operatorname{conv}}\left(A \cup \bigcup_{|\lambda|=1} \lambda C\right) .
$$

Observe that if $p_{X}$ denotes the projection on $X$, and if $B_{X}$ is the closed unit ball of $X$, then $p_{X}(A)=B_{X}$ and $p_{X}(C) \subset B_{X}$; therefore $P_{X}(W) \subset B_{X}$. In particular, whenever $|t| \leq\|x\|$, then

$$
(x, t) \in W \Rightarrow(x, t) \in A,
$$

and therefore $\|(x, t)\|_{W}=\|x\|$. This implies that the norm $\|.\|_{W}$ coincides with $\|$. on $X$.

Let $T$ be an $\mathbb{R}$-linear isometry on $T$ for $\|\cdot\|_{w}$. We intend to prove (1) to (4) for $T$.

We note that $C$ as well as all its rotations $\lambda C,|\lambda|=1$, are faces of $W$. We distinguish two types of points in $\delta W:$ A) points interior to a segment $I$ contained in $\delta W$, whose length (with respect to the $W$-norm) is at least 0.1 , and the limits of such points; B) all other points.

As these types are $\mathbb{R}$-linearly metrically defined, they are preserved by $T$. On the other hand it is easy to see that the points of type A) cover all of $\delta W$ except the relative interiors of the faces $\lambda C$. Hence $T\left(x_{0}, 2\right)$ belongs to some $\lambda C$ with $|\lambda|=1$. Replacing $T$ by $\lambda^{-1} T$ we may assume that $T\left(x_{0}, 2\right) \in C$. Since $T$ maps the face $C$ onto a face of $W$ we have $T C=C$. To prove that $T$ maps $X$ onto $X$, let $x \in X$ with $p(x) \leq 1$. We have

$$
T(x, 0)=T\left(\left(x+x_{0}, 2\right)-\left(x_{0}, 2\right)\right)=T\left(x+x_{0}, 2\right)-T\left(x_{0}, 2\right) \in C-C \subset X,
$$

and as $\{x: p(x) \leq 1\}$ contains a ball in $X$ this is true for all $x \in X$, i.e. $T X \subset X$; by symmetry, using $T^{-1}, T X=X$. Because the $\|\cdot\|_{W}$-norm agrees with $\|\cdot\|$ on $X$, it follows that $T_{\mid X}$ is a $\|$.$\| -isometry. Since T C=C$ we claim that $T$ maps $\left(x_{0}, 2\right)$ onto itself. Indeed otherwise let $D$ be the line joining $\left(x_{0}, 2\right)$ to $T\left(x_{0}, 2\right)$. Since

$$
C=\left(x_{0}, 2\right)+\{(x, 0): p(x) \leq 1\},
$$

we have that $\left(x_{0}, 2\right)$ is the center of the segment $C \cap D$. Likewise, since

$$
C=T C=T\left(x_{0}, 2\right)+\{(T x, 0): p(x) \leq 1\},
$$


$T\left(x_{0}, 2\right)$ is the center of $C \cap D$. This contradicts the hypothesis that $T\left(x_{0}, 2\right) \neq$ $\left(x_{0}, 2\right)$. Therefore $T_{\mid X}$ maps $\left(x_{0}, 2\right)$ onto $\left(x_{0}, 2\right)$, proving the claim.

It follows that $T_{\mid X}$ also maps the unit ball for $p($.$) onto itself. Therefore T_{\mid X}$ is an isometry for $p($.$) as well.$

Finally we shall prove that $T x_{0}=x_{0}$. It will then follow that $T(0,1)=(0,1)$, which will conclude the proof of the lemma. So assume towards a contradiction that $T x_{0} \neq x_{0}$. Let

$$
x=T^{-1} \frac{x_{0}-T x_{0}}{\left\|x_{0}-T x_{0}\right\|},
$$

and note that $x$ is a normalized vector in $X$. It follows that $(x, 1)$ belongs to $A$ and therefore to $W$. On the other hand, we have

$$
T(0,1)=\frac{T\left(x_{0}, 2\right)-T\left(x_{0}, 0\right)}{2}=\left(\frac{x_{0}-T x_{0}}{2}, 1\right),
$$

so

$$
T(x, 1)=(T x, 0)+T(0,1)=\left(\frac{x_{0}-T x_{0}}{\left\|x_{0}-T x_{0}\right\|}+\frac{x_{0}-T x_{0}}{2}, 1\right),
$$

and since

$$
\left\|\frac{x_{0}-T x_{0}}{\left\|x_{0}-T x_{0}\right\|}+\frac{x_{0}-T x_{0}}{2}\right\|=1+\frac{\left\|x_{0}-T x_{0}\right\|}{2}>1
$$

we have that

$$
\|T(x, 1)\|_{w}=1+\frac{\left\|x_{0}-T x_{0}\right\|}{2}>1 .
$$

From this it follows that $T(x, 1)$ does not belong to $W$, a contradiction with the fact that $(x, 1)$ belongs to $W$. Therefore $T x_{0}=x_{0}$ and the result is proved.

Proposition 40. Let $(X,\|\cdot\|)$ be a complex Banach space, $x_{0}$ a nonzero element of $X, p($.$) a continuous norm on (X,\|\|),. G_{1}$ the group of all real isometries of $(X,\|\|$.$) and G_{2}$ the group of all real isometries $T$ of $(X, p()$.$) such that T x_{0}$, Tix $x_{0}$ and $x_{0}$ are mutually $\mathbb{C}$-linearly dependent. Let $\|\cdot\|_{w}$ be the norm defined in Lemma 39 on $Y=X \oplus \mathbb{C}$ and $G$ be the group of real isometries of $\left(Y,\|\cdot\|_{w}\right)$. Then the map $\alpha$ defined by $\alpha(T)=T_{\mid X}$ defines a group isomorphism from $G$ onto $G_{1} \cap G_{2}$.

Proof. We claim that for any $T \in G_{2}$, and for $\lambda \in \mathbb{C}_{1}$ such that $T x_{0}=\lambda x_{0}$, we have that $T i x_{0}= \pm \lambda i x_{0}$. Indeed let $\lambda \in \mathbb{C}_{1}$ be such that $T x_{0}=\lambda x_{0}$ and let $\mu \in \mathbb{C}_{1}$ be such that $T i x_{0}=\mu x_{0}$. Then since $T e^{i \theta}$ is a $p($.$) -isometry for any \theta$,

$$
p\left(x_{0}\right)=p\left(T e^{i \theta} x_{0}\right)=|\cos \theta \lambda+\sin \theta \mu| p\left(x_{0}\right) .
$$

It follows easily that $\mu= \pm i \lambda$, and this proves the claim.

We now study the map $\alpha$. The inverse of $\alpha$, which we already denote by $\alpha^{-1}$ to avoid excessive notation, will be given by the following formula. If $T \in G_{1} \cap G_{2}$, and $\lambda \in \mathbb{C}_{1}$ is such that $T x_{0}=\lambda x_{0}$, then

$$
\alpha^{-1}(T)=T \oplus \lambda I d_{\mathbb{C}},
$$

if $T i x_{0}=\lambda i x_{0}$, and

$$
\alpha^{-1}(T)=T \oplus \lambda c_{\mathbb{C}}
$$

if $\operatorname{Tix}_{0}=-\lambda i x_{0}$.

We note that if $T \in G$, then by Lemma 39. $T_{\mid X}$ belongs to $G_{1} \cap G_{2}$, and since $\alpha(T U)=\alpha(T) \alpha(U)$ for $T, U \in G, \alpha$ is a group homomorphism.

Conversely if $T \in G_{1} \cap G_{2}$, let $\tilde{T}=\alpha^{-1}(T)$. We wish to prove that $\tilde{T} \in G$. 
Let $\lambda \in \mathbb{C}_{1}$ be such that $T x_{0}=\lambda x_{0}$. If $T i x_{0}=\lambda i x_{0}$, then $\tilde{T}=T \oplus \lambda I d_{\mathbb{C}}$. From the fact that for any $\mu \in \mathbb{C}, T\left(\mu x_{0}\right)=\lambda \mu x_{0}$, and that $B_{p}=\{(x, 0): p(x) \leq 1\}$ is $T$-invariant, we have

$$
\tilde{T}(\mu C)=\left(T \mu x_{0}, 2 \lambda \mu\right)+T \mu B_{p}=\left(\lambda \mu x_{0}, 2 \lambda \mu\right)+B_{p}=\lambda \mu\left(\left(x_{0}, 2\right)+B_{p}\right)=\lambda \mu C .
$$

Therefore $\tilde{T}\left(\bigcup_{\mu \in \mathbb{C}_{1}} \mu C\right)=\bigcup_{\mu \in \mathbb{C}_{1}} \mu C$. Furthermore it is clear that $\tilde{T}(A)=A$, so finally $\tilde{T} W=W$ and $\tilde{T}$ is an isometry for $\|\cdot\|_{W}$, that is, $\tilde{T} \in G$.

Now if $T i x_{0}=-\lambda i x_{0}$, then $\tilde{T}=T \oplus \lambda c_{\mathbb{C}}$. For any $\mu \in \mathbb{C}, T\left(\mu x_{0}\right)=\lambda \bar{\mu} x_{0}$, and $B_{p}$ is still $T$-invariant, so we have

$$
\tilde{T}(\mu C)=\left(T \mu x_{0}, 2 \lambda \bar{\mu}\right)+T \mu B_{p}=\left(\lambda \bar{\mu} x_{0}, 2 \lambda \bar{\mu}\right)+B_{p}=\lambda \bar{\mu}\left(\left(x_{0}, 2\right)+B_{p}\right)=\lambda \bar{\mu} C .
$$

Therefore $\tilde{T}\left(\bigcup_{\mu \in \mathbb{C}_{1}} \mu C\right)=\bigcup_{\mu \in \mathbb{C}_{1}} \mu C$. Furthermore it is clear that for such a $T$ we have $\tilde{T}(A)=A$ as well, so finally $\tilde{T} W=W$ and $\tilde{T}$ is an isometry for $\|\cdot\|_{W}$, that is, $\tilde{T} \in G$.

These two cases prove that $\alpha^{-1}$ takes values in $G$.

Now it is clear that $\alpha \alpha^{-1}=I d_{G_{1} \cap G_{2}}$. It only remains to check that $\alpha^{-1} \alpha=I d_{G}$. Let $T \in G$, and since $T_{\mid X}$ belongs to $G_{1} \cap G_{2}$, let $\lambda \in \mathbb{C}$ be such that $T x_{0}=\lambda x_{0}$. So by Lemma $39(4), T(0,1)=(0, \lambda)$. Now we have that Tix $x_{0}= \pm \lambda i x_{0}$.

If $T i x_{0}=\lambda i x_{0}$, then by Lemma 39 (4) applied to $T i$, which is also an $\mathbb{R}$-linear isometry, $T(0, i)=(0, \lambda i)$. So $T(0, z)=(0, \lambda z)=\left[\alpha^{-1}\left(T_{\mid X}\right)\right](0, z)$ for all $z \in \mathbb{C}$, that is, $T_{\mathbb{C}}=\lambda I d_{\mathbb{C}}$. This means that $\alpha^{-1}\left(T_{\mid X}\right)=T_{\mid X} \oplus \lambda I d_{\mathbb{C}}=T$.

If on the contrary, $T i x_{0}=-\lambda i x_{0}$, then by Lemma 39 (4) applied to $T i, T(0, i)=$ $(0,-\lambda i)$. So $T(0, z)=(0, \lambda \bar{z})=\left[\alpha^{-1}\left(T_{\mid X}\right)\right](0, z)$ for all $z \in \mathbb{C}$, that is, $T_{\mid \mathbb{C}}=\lambda c_{\mathbb{C}}$. This means that $\alpha^{-1}\left(T_{\mid X}\right)=T_{\mid X} \oplus \lambda c_{\mathbb{C}}=T$.

We have therefore proved in all cases that

$$
\alpha^{-1}\left(T_{\mid X}\right)=T \text {. }
$$

Finally the proof that $\alpha$ is a group isomorphism with inverse $\alpha^{-1}$ is complete.

In the previous proposition it may happen that $X$ admits a subspace $E$ such that $X=E \oplus i E$. In that case we shall write $\mathbb{C}=\mathbb{R} \oplus i \mathbb{R}$ and $Y=X \oplus \mathbb{C}=$ $(E \oplus \mathbb{R}) \oplus i(E \oplus \mathbb{R})$. Therefore $Y$ is isomorphic to the complexification of $E \oplus \mathbb{R}$. Furthermore we claim that, equipped with $\|.\|_{W}$ defined in Lemma 39, $Y$ is regular provided $x_{0}$ was chosen in $E$ and provided that $X=E \oplus i E$ was regular both for $\|$.$\| and p($.$) . To see this, assume that x_{0} \in E$ and that the conjugation map $c_{X}$ on $X$ is an isometry for $\|$.$\| and for p($.$) . Denote by c=c_{X} \oplus c_{\mathbb{C}}$ the conjugation map on $Y$. Then for any $\mu \in \mathbb{C}$,

$$
c(\mu C)=\left(c_{X}\left(\mu x_{0}\right), 2 \bar{\mu}\right)+c_{X}\left(B_{p}\right)=\left(\bar{\mu} x_{0}, 2 \bar{\mu}\right)+B_{p}=\bar{\mu}\left(\left(x_{0}, 2\right)+B_{p}\right)=\bar{\mu} C .
$$

Therefore $\bigcup_{\mu \in \mathbb{C}_{1}} \mu C$ is invariant by $c$. Since it is also clear that $c(A)=A$, this implies that $W$ is invariant by $c$. This finally proves the claim that $c$ is an isometry on $Y$ for $\|\cdot\|_{w}$.

To sum up, if $x_{0} \in E$ and the conjugation map $c_{X}$ on $X$ is an isometry for $\|$.$\| and for p($.$) , then we have that c$ is an isometry on $Y$ for $\|.\|_{W}$, and we may therefore talk about conjugation isometries on $\left(Y,\|\cdot\|_{w}\right)$.

Corollary 41. Let $(X,\|\|$.$) be a complex Banach space, and let p($.$) be a continuous$ norm on $(X,\|\cdot\|)$. Then there is a norm $\|\cdot\|_{w}$ on $Y=X \oplus \mathbb{C}$ such that $\|\cdot\|_{w}$ and 
\|.\| coincide on $X$ and such that:

(1) If every real isometry on $X$ for $\|$.$\| and for p($.$) is trivial, then every real$ isometry on $Y$ for $\|.\|_{w}$ is trivial.

(2) If $X$ is regular for $\|$.$\| and p($.$) , then Y$ is regular for $\|.\|_{w}$, and if furthermore every real isometry on $X$ for $\|$.$\| and for p($.$) is a trivial or a con-$ jugation isometry, then every real isometry on $Y$ for $\|\cdot\|_{w}$ is a trivial or a conjugation isometry.

Proof. In case (1), $G_{1} \cap G_{2}$ is the group of trivial isometries on $X$ for any choice of $x_{0}$. Therefore Proposition 40 implies that $G$ is the group of trivial real isometries on $Y$.

In case (2), assume that we picked $x_{0}$ in the real part $E$ of $X=E \oplus i E$. When $T$ is trivial, that is $T=\lambda I d$, then $T x_{0}=\lambda x_{0}$ and $T i x_{0}=\lambda i x_{0}$. When $T$ is a conjugation isometry, that is $T=\lambda c_{X}$, then $T x_{0}=\lambda x_{0}$ as well and $T i x_{0}=-\lambda i x_{0}$. This means that $G_{1} \cap G_{2}$ is the group of trivial or conjugation isometries on $X$. Applying Proposition 40 for this choice of $x_{0}$, for any isometry $T$ on $Y$, either $T_{\mid X}=\lambda I d$, and then $T=\alpha^{-1}\left(T_{\mid X}\right)=T \oplus \lambda I d_{\mathbb{C}}$, so $T$ is the trivial isometry $\lambda I d$; or $T_{\mid X}=\lambda c_{X}$, and then $T=\alpha^{-1}\left(T_{\mid X}\right)=T \oplus \lambda c_{\mathbb{C}}$, so $T$ is the conjugation isometry $\lambda c$.

The following fact, due to Pličko [16], was cited and used in [11].

Proposition 42 (Pličko [16]). For any real (resp. complex) Banach space $X$ there is a set $\Gamma$ and a continuous, $\mathbb{R}$-linear (resp. $\mathbb{C}$-linear) injective map $J$ from $X$ into $\ell_{\infty}(\Gamma, \mathbb{R})$ (resp. $\ell_{\infty}(\Gamma, \mathbb{C})$ ) such that the closure of $J(X)$ contains $c_{0}(\Gamma, \mathbb{R})$ (resp. $\left.c_{0}(\Gamma, \mathbb{R})\right)$.

In the following theorems we shall consider a complex Banach space $X$ and a complex Banach space $Y$ such that $X \subset Y$ and $\operatorname{dim} Y / X=1$, that is, $Y=X \oplus \mathbb{C}$. When $X$ is regular with respect to the decomposition $X=E \oplus i E$, we shall see as before $Y$ as regular with respect to the decomposition $Y=(E \oplus \mathbb{R}) \oplus i(E \oplus \mathbb{R})$ under an appropriate norm. For clarity we state two different theorems for the case of spaces with only trivial isometries and for the case of spaces with only trivial and conjugation isometries, although their proofs are similar. Dimensions are considered over $\mathbb{C}$.

Theorem 43. For any complex Banach space $X$ of dimension at least 1 , there is a complex Banach space $Y$ with $X \subset Y$ and $\operatorname{dim} Y / X=1$ such that $Y$ has only trivial real isometries.

Proof. This imitates the proof of [11, Theorem 1. Let $Y=X \oplus \mathbb{C}$. If $\operatorname{dim} X=1$, then the proof holds from Lemma 34, since we may assume that the norm on $X \simeq \mathbb{C}$ is the modulus, and the norm $\|\cdot\|$ on $\mathbb{C}^{2}$ from Lemma 34 satisfies $\|(x, 0)\|=|x|$, for all $x \in \mathbb{C}$. So we may assume that $\operatorname{dim} X \geq 2$. Let $J: X \rightarrow \ell_{\infty}(\Gamma, \mathbb{C})$ be an injective map given by Proposition 42, Let $E:=\overline{J(X)} \subset \ell_{\infty}(\Gamma, \mathbb{C})$. We claim that there is a continuous norm $\tilde{p}$ on $E$ such that $(E, \tilde{p})$ has only trivial isometries. If $\operatorname{dim} X=2$, then $\operatorname{dim} E=2$ and therefore the claim holds by Lemma 34, so we may assume that $\operatorname{dim} X \geq 3$, so $|\Gamma| \geq 3$. Fix $\gamma \in \Gamma$. Let $\left\|\left|\|\mid\|\right.\right.$ be the norm $\|\cdot\|_{1}$ on $\{e \in E: e(\gamma)=0\} \subset \ell_{\infty}(\Gamma \backslash\{\gamma\}, \mathbb{C})$ given by Proposition 35. We then have

$$
E \simeq\{e \in E: e(\gamma)=0\} \oplus_{\infty} \mathbb{C},
$$


so by Corollary 41, there is a continuous norm $\tilde{p}$ on $E$ such that $(E, \tilde{p})$ has only trivial isometries, and this proves the claim.

We then define a continuous norm $p$ on $X$ by

$$
p(x)=\tilde{p}(J x), \quad x \in X .
$$

Evidently $(J X, \tilde{p})$ has only trivial isometries. Then for any isometry $T$ on $X$ for $p$, the map $\tilde{T}$ defined on $J X$ by $\tilde{T}(J x)=J T x$ is easily an isometry on $J X$ for $p$, and therefore a trivial isometry, so $T$ is a trivial isometry. Therefore $(X, p)$ has only trivial isometries.

Hence, again by Corollary 41 , there is a norm on $Y=X \oplus \mathbb{C}$, with only trivial isometries, which coincides with $\|$.$\| on X$.

Theorem 44. For any regular complex Banach space $X$, there is a regular complex Banach space $Y$, with $X \subset Y$ and $\operatorname{dim} Y / X=1$, such that $Y$ has only trivial and conjugation real isometries.

Proof. Let $Y=X \oplus \mathbb{C}$. If $\operatorname{dim} X=0$, then the result is trivial, so we may assume $\operatorname{dim} X \geq 1$. Write $X=Z \oplus i Z$, and by Proposition 42 define an injective map $j: Z \rightarrow \ell_{\infty}(\Gamma, \mathbb{R})$ such that $c_{0}(\Gamma, \mathbb{R}) \subset Z$. Define $J: X \rightarrow \ell_{\infty}(\Gamma, \mathbb{C})$ by $J(x+i y)=$ $j x+i j y$. Then $J$ is injective and $c_{0}(\Gamma, \mathbb{C}) \subset E \subset l_{\infty}(\Gamma, \mathbb{C})$, where $E:=\overline{J X}$. We claim that there is a continuous norm $\tilde{p}$ on $E$ for which $E$ is regular and such that $(E, \tilde{p})$ has only trivial and conjugation isometries. If $\operatorname{dim} X=1$, that is $\operatorname{dim} E=1$, then this is obvious, so we may assume that $\operatorname{dim} X \geq 2$. Fix $\gamma \in \Gamma$. Let $\||\cdot \||$ be the norm $\|\cdot\|_{2}$ on $\{e \in E: e(\gamma)=0\} \subset \ell_{\infty}(\Gamma \backslash\{\gamma\}, \mathbb{C})$ given by Proposition 35, Note that the conjugation map on $\{e \in E: e(\gamma)=0\}$ is an isometry for that norm, so that space is regular. We then have

$$
E \simeq\{e \in E: e(\gamma)=0\} \oplus_{\infty} \mathbb{C},
$$

so by Corollary 41 (2), there is a continuous norm $\tilde{p}$ on $E$ for which $E$ is regular and such that $(E, \tilde{p})$ has only trivial and conjugation isometries; that is, the claim is proved. Note that $J X$ is a dense subspace of $E$ and is stable by the conjugation map; $(J X, \tilde{p})$ is canonical and admits only trivial and conjugation isometries.

We then define a continuous norm $p$ on $X$ by

$$
p(x)=\tilde{p}(J x), \quad x \in X .
$$

For any $u, v \in Z$,

$$
p(u-i v)=\tilde{p}(j u-i j v)=\tilde{p}(j u+i j v)=p(u+i v),
$$

so the conjugation map is an isometry and $(X, p)$ is canonical. Furthermore for any isometry $T$ on $X$ for $p$, the map $\tilde{T}$ defined on $J X$ by $\tilde{T}(J x)=J T x$ is an isometry on $J X$ for $p$. If it is a trivial isometry, then $T$ is a trivial isometry. If it is a conjugation isometry, that is, for $u, v \in Z$,

$$
J T(u+i v)=\tilde{T}(j u+i j v)=\lambda(j u-i j v)=J(\lambda(u-i v)),
$$

then $T(u+i v)=\lambda(u-i v)$ and so $T$ is a conjugation isometry.

Therefore we have proved that $(X, p)$ has only trivial or conjugation isometries.

Hence, again by Corollary 41 (2), there is a norm on $Y=X \oplus \mathbb{C}$, which coincides with $\|$.$\| on X$, for which $Y$ is canonical and admits only trivial and conjugation isometries. 
Corollary 45. For any complex Banach space $X$ of dimension at least 2 , there is an equivalent complex norm on $X$ for which $X$ has only trivial real isometries.

Corollary 46. For any complexification $X$ of a real Banach space, there is an equivalent regular complex norm on $X$ for which $X$ has exactly trivial and conjugation real isometries.

\section{ISOMETRIES ON REAL HI SPACES}

It may be interesting to conclude this article by noting that isometries on the real HI space of Gowers and Maurey, or more generally, on spaces such that every operator is a strictly singular perturbation of a multiple of the identity, have specific properties under any equivalent norm. This was obtained in [17] in the complex case.

Denote by $\hat{X}$ the complexification of a real Banach space $X$. As we know, we may write $\hat{X}=\{x+i y: x, y \in X\}$. Let $A, B \in L(X)$. Then

$$
(A+i B)(x+i y):=A x-B y+(A y+B x)
$$

defines an operator $A+i B \in L(\hat{X})$. Conversely, given $T \in L(\hat{X})$, if we put $T(x+i 0):=A x+i B x$, then we obtain $A, B \in L(X)$ such that $T=A+i B$. We write $\hat{T}=T+i 0$ for $T \in \mathcal{L}(X)$.

Let $T \in L(X)$. We recall that the group $\left(e^{t T}\right)_{t \in \mathbb{R}}$ has growth order $k \in \mathbb{N}$ if $\left\|e^{t T}\right\|=\sigma\left(|t|^{k}\right)$ as $|t| \rightarrow+\infty$. We also recall that an invertible operator $T \in L(X)$ is polynomially bounded of order $k \in \mathbb{N}$ if $\left\|T^{n}\right\|=\sigma\left(n^{k}\right)$ as $|n| \rightarrow+\infty$. In [17, Theorem 3.2, it is proved that:

Proposition 47 (17). Let $X$ be a complex Banach space and $T \in L(X)$ such that there exists $\lambda \in \mathbb{C}$ with $T-\lambda I \in S(X)$ and the group $\left(e^{t T}\right)_{t \in \mathbb{R}}$ has growth order $k \in \mathbb{N}$. Then $(T-\lambda I)^{k}$ is a compact operator.

The result in [17] is stated for complex HI spaces, but the proof only uses the fact that complex HI spaces satisfy the $\lambda I d+S$ property. So by using this proposition instead of [17. Theorem 3.2, we can prove in a similar way to [17] Theorem 3.5 the following result:

Proposition 48. Suppose that $X$ is a complex Banach space with the $\lambda I d+S$ property and that $T \in L(X)$ is an invertible operator, polynomially bounded of order $k \in \mathbb{N}$. Let $\lambda \in \mathbb{C}$ such that $T-\lambda I \in S(X)$. Then $(T-\lambda I)^{k}$ is a compact operator.

We deduce:

Proposition 49. Suppose that $X$ is a real Banach space with the $\lambda I d+S$ property and that $T \in L(X)$ is an isometry. Then $T$ is of the form $\pm I d+K, K$ compact.

Proof. Let $T$ be an isometry on $X$, and $a \in \mathbb{R}, S$ strictly singular be such that $T=a I d+S$. Clearly $a= \pm 1$. Let $\hat{X}$ be the complexification of $X$. Using [8], Proposition 2.6, it is easy to check that $\hat{X}$ has the $\lambda I d+S$ property. Consider $\hat{T}=T+i .0 \in L(\hat{X})$. Notice that $\hat{T}$ is an isomorphism from $\hat{X}$ onto $\hat{X}$. Moreover, $\hat{T}^{n}=T^{n}+i .0, \quad \forall n \in \mathbb{N}$ and thus $\left\|\hat{T}^{n}\right\|$ is bounded. In particular, $\hat{T}$ is polynomially bounded of order 1 .

Now notice that $\hat{T}-a \hat{I d}=(T-a I d)+i .0$. Thus by [8], Proposition 2.6, $\hat{T}-a \hat{I d} \in S(\hat{X})$. Therefore according to Proposition 48, $\hat{T}-\lambda I d$ is a compact operator. So by $[8$, Proposition 2.4, T-aId is also a compact operator. 
Question 50. Let $X$ be a real H.I. Banach space such that every operator is of the form $\lambda I d+\mu J+S$, where $J^{2}=-I d$. Does it follow that every isometry is of the form $\lambda I d+\mu J+K, K$ compact?

In this direction, it is natural to ask whether the complexification of a real HI space is always HI. By the proof of [6] Proposition 35, this is always the case when every operator on a subspace $Y$ of $X$ is of the form $\lambda i_{Y X}+s, \lambda \in \mathbb{R}, s$ strictly singular. Observe that by 8 Proposition 3.16, if a real Banach space $X$ is such that $\mathcal{L}(X) / \mathcal{S}(X)$ is isomorphic to $\mathbb{C}$ or $\mathbb{H}$, then the complexification $\hat{X}$ of $X$ is decomposable. Therefore if $\hat{X}$ is HI for some real space $X$, then every subspace of $X$ must have the $\lambda I d+S$ property.

\section{Appendix}

We give the proof of two lemmas used in Section 2. They are inspired by [4, Theorem 7.4, page 72 and by the properties of Day's norm on $c_{0}$ (4, page 69).

Lemma 51. Let $Y$ be a Banach space with an LUR norm, let $1 \leq p<+\infty$, and let $X=\ell_{p}(Y)$. Then there exists an equivalent LUR norm on $X$ for which any map $T$ defined on $X$ by $T\left(\left(y_{n}\right)_{n \in \mathbb{N}}\right)=\left(\epsilon_{n} y_{\sigma(n)}\right)_{n \in \mathbb{N}}$, where $\epsilon_{n}= \pm 1$ for all $n \in \mathbb{N}$ and $\sigma$ is a permutation on $\mathbb{N}$, is an isometry.

Proof. Fix an equivalent LUR norm $\|\cdot\|$ on $Y$, and let $\|\cdot\|=\|\cdot\|_{p}$ be the corresponding $\ell_{p}$-norm on $X$, when $p>1$. When $p=1$, let $\|\cdot\|_{1}$ denote the corresponding $\ell_{1}$-norm, $\|\cdot\|_{2}$ denote the corresponding $\ell_{2}$-norm (via the canonical "identity" map from $\ell_{1}$ into $\ell_{2}$ ), and let $\|$.$\| be the equivalent norm defined on X$ by

$$
\|x\|^{2}=\|x\|_{1}^{2}+\|x\|_{2}^{2} .
$$

To prove that $\|\cdot\|$ is LUR, let $x=\left(y_{k}\right)_{k} \in X$ and $x_{n}=\left(y_{n, k}\right)_{k} \in X$ with $\lim _{n}\left\|x_{n}\right\|=$ $\|x\|$ and $\lim _{n}\left\|x+x_{n}\right\|=2\|x\|$. We need to prove that $\lim _{n} x_{n}=x$.

We first assume that $p=1$. We have that

$$
\lim _{n}\left(2\|x\|^{2}+2\left\|x_{n}\right\|^{2}-\left\|x+x_{n}\right\|^{2}\right)=0 .
$$

Using 4, Fact 2.3 , p. 45, (1) implies that

$$
\lim _{n}\left(2\|x\|_{1}^{2}+2\left\|x_{n}\right\|_{1}^{2}-\left\|x+x_{n}\right\|_{1}^{2}\right)=0
$$

and

$$
\lim _{n}\left(2\|x\|_{2}^{2}+2\left\|x_{n}\right\|_{2}^{2}-\left\|x+x_{n}\right\|_{2}^{2}\right)=0 .
$$

By [4], Fact 2.3 again, (3) implies, for all $k \in \mathbb{N}$, that

$$
\lim _{n}\left(2\left\|y_{k}\right\|^{2}+2\left\|y_{n, k}\right\|^{2}-\left\|y_{k}+y_{n, k}\right\|^{2}\right)=0,
$$

whence, since the norm on $Y$ is LUR, by [4], Proposition 1.2, p. 42,

$$
\lim _{n} y_{n, k}=y_{k}, \forall k \in \mathbb{N}
$$

and from (2) we have (see 4, p. 42),

$$
\lim _{n}\left\|x_{n}\right\|_{1}=\|x\|_{1} .
$$

Now assume $p>1$. We have that

$$
\lim _{n}\left\|x_{n}\right\|_{p}=\|x\|_{p},
$$


which means that

$$
\lim _{n} \sum_{k}\left\|y_{n, k}\right\|^{p}=\sum_{k}\left\|y_{k}\right\|^{p}
$$

Let $|\cdot|_{p}$ also denote the norm on $\ell_{p}$. Since

$$
\begin{gathered}
\left\|x_{n}+x\right\|=\left|\left(\left\|y_{n, k}+y_{k}\right\|\right)_{k}\right|_{p} \leq\left|\left(\left\|y_{n, k}\right\|+\left\|y_{k}\right\|\right)_{k}\right|_{p} \\
\leq\left|\left(\left\|y_{n, k}\right\|\right)_{k}\right|_{p}+\left|\left(\left\|y_{k}\right\|\right)_{k}\right|_{p}=\left\|x_{n}\right\|+\|x\|
\end{gathered}
$$

and both $\left\|x_{n}+x\right\|$ and $\left\|x_{n}\right\|+\|x\|$ converge to $2\|x\|$, we deduce that

$$
\lim _{n}\left|\left(\left\|y_{n, k}\right\|+\left\|y_{k}\right\|\right)_{k}\right|_{p}=2\left|\left(\left\|y_{k}\right\|\right)_{k}\right|_{p}
$$

Since $|\cdot|_{p}$ is LUR on $\ell_{p}$, we deduce from (7) and (8) that $\lim _{n}\left|\left(\left\|y_{n, k}\right\|-\left\|y_{k}\right\|\right)_{k}\right|_{p}=0$; in particular

$$
\forall k \in \mathbb{N}, \lim _{n}\left\|y_{n, k}\right\|=\left\|y_{k}\right\|
$$

Since $\left\|x+x_{n}\right\|$ converges to $2\|x\|$ we also have

$$
\lim _{n} \sum_{k}\left\|y_{n, k}+y_{k}\right\|^{p}=2^{p} \sum_{k}\left\|y_{k}\right\|^{p} .
$$

Fix $k_{0} \in \mathbb{N}$ and $\epsilon>0$. We may find some $k_{1}>k_{0}$ such that

$$
\sum_{k \geq k_{1}}\left\|y_{k}\right\|^{p}<\epsilon
$$

Therefore by (7), (9), and (11), for $n$ large enough,

$$
\sum_{k \geq k_{1}}\left\|y_{n, k}\right\|^{p}<2 \epsilon .
$$

Using (9), (11) and (12), we deduce that for $n$ large enough,

$$
\sum_{k}\left\|y_{n, k}+y_{k}\right\|^{p}<2^{p} \sum_{k \neq k_{0}, k<k_{1}}\left\|y_{k}\right\|^{p}+\epsilon+2^{p} .3 \epsilon+\left\|y_{n, k_{0}}+y_{k_{0}}\right\|^{p},
$$

while by (10) and (11), for $n$ large enough,

$$
\sum_{k}\left\|y_{n, k}+y_{k}\right\|^{p}>2^{p} \sum_{k \neq k_{0}, k<k_{1}}\left\|y_{k}\right\|^{p}+2^{p}\left\|y_{k_{0}}\right\|^{p}-2^{p} \epsilon-\epsilon .
$$

From (13) and (14) we deduce that for $n$ large enough,

$$
2^{p}\left\|y_{k_{0}}\right\|^{p}<\left(2+4.2^{p}\right) \epsilon+\left\|y_{n, k_{0}}+y_{k_{0}}\right\|^{p},
$$

and we deduce, using also (9), that

$$
\lim _{n}\left\|y_{n, k_{0}}+y_{k_{0}}\right\|=2\left\|y_{k_{0}}\right\| \text {. }
$$

From (9) and (15), and from the fact that the norm on $Y$ is LUR, it follows that

$$
\forall k \in \mathbb{N}, \lim _{n} y_{n, k}=y_{k}
$$


Going back to the general case, fix $\epsilon>0$ and let $k_{1} \in \mathbb{N}$ be such that $\sum_{k>k_{1}}\left\|y_{k}\right\|^{p}$ $<\epsilon$. Then

$$
\begin{aligned}
& \left\|x-x_{n}\right\|_{p}^{p}=\sum_{k<k_{1}}\left\|y_{k}-y_{n, k}\right\|^{p}+\sum_{k \geq k_{1}}\left\|y_{k}-y_{n, k}\right\|^{p} \\
& \leq \sum_{k<k_{1}}\left\|y_{k}-y_{n, k}\right\|^{p}+2^{p} \sum_{k \geq k_{1}}\left\|y_{k}\right\|^{p}+2^{p} \sum_{k \geq k_{1}}\left\|y_{n, k}\right\|^{p} \\
& =\sum_{k<k_{1}}\left\|y_{k}-y_{n, k}\right\|^{p}+2^{p}\left(2 \sum_{k \geq k_{1}}\left\|y_{k}\right\|^{p}+\left(\left\|x_{n}\right\|_{p}^{p}-\|x\|_{p}^{p}\right)+\sum_{k<k_{1}}\left(\left\|y_{k}\right\|^{p}-\left\|y_{n, k}\right\|^{p}\right)\right) .
\end{aligned}
$$

So by (4) and (5) when $p=1$, or by (6) and (16) when $p>1$, we obtain that $\left\|x-x_{n}\right\|_{p}^{p}<3.2^{p} \epsilon$ for $n$ large enough.

Lemma 52. Let $Y$ be a Banach space with an LUR norm and let $X=c_{0}(Y)$. Then there exists an equivalent LUR norm on $X$ for which any map $T$ defined on $X$ by $T\left(\left(y_{n}\right)_{n \in \mathbb{N}}\right)=\left(\epsilon_{n} y_{\sigma(n)}\right)_{n \in \mathbb{N}}$, where $\epsilon_{n}= \pm 1$ for all $n \in \mathbb{N}$ and $\sigma$ is a permutation on $\mathbb{N}$, is an isometry.

Let $|\cdot|_{D}$ denote the equivalent Day's norm on $c_{0}$; that is, for $x=\left(x_{n}\right)_{n} \in c_{0}$,

$$
|x|_{D}=\sup \left(\sum_{i=1}^{k} x_{n_{i}}^{2} / 4^{i}\right)^{1 / 2},
$$

where the sup is taken over $k \in \mathbb{N}$ and all $k$-tuples $\left(n_{1}, \ldots, n_{k}\right)$ of distinct elements of $\mathbb{N}$. Let $\|$.$\| denote the corresponding norm on X=c_{0}(Y)$; therefore for $x=$ $\left(y_{k}\right)_{k} \in X$,

$$
\|x\|=\sup \left(\sum_{i=1}^{k}\left\|y_{n_{i}}\right\|^{2} / 4^{i}\right)^{1 / 2} .
$$

Let $\|\cdot\|_{\infty}$ denote the sup norm on $X,\|x\|_{\infty}=\sup _{k}\left\|y_{k}\right\|$. Note that isomorphisms associated to a permutation on $\mathbb{N}$ and a sequence of signs are isometries on $X$ for $\|$.$\| . It remains to prove that \|$.$\| is LUR. Let x=\left(y_{k}\right)_{k} \in X$ and $x_{n}=\left(y_{n, k}\right)_{k} \in X$ be such that

and

$$
\lim _{n}\left\|x_{n}\right\|=\|x\|
$$

$$
\lim _{n}\left\|x+x_{n}\right\|=2\|x\| .
$$

We need to prove that $\lim _{n}\left\|x-x_{n}\right\|=0$ or equivalently $\lim _{n}\left\|x-x_{n}\right\|_{\infty}=0$. Since $\left(x_{n}\right)_{n}$ is arbitrary satisfying (17) and (18), it is enough to prove that some subsequence of $\left(x_{n}\right)_{n}$ satisfies $\lim _{n}\left\|x-x_{n}\right\|_{\infty}=0$.

Since, by elementary properties of $|\cdot|_{D}$,

$$
\left\|x+x_{n}\right\|=\left|\left(\left\|y_{k}+y_{n, k}\right\|\right)_{k}\right|_{D} \leq\left|\left(\left\|y_{k}\right\|+\left\|y_{n, k}\right\|\right)_{k}\right|_{D} \leq\|x\|+\left\|x_{n}\right\|,
$$

we deduce from (17) and (18) that

$$
\lim _{n}\left|\left(\left\|y_{k}\right\|+\left\|y_{n, k}\right\|\right)_{k}\right|_{D}=2\left|\left(\left\|y_{k}\right\|\right)_{k}\right|_{D}
$$

Since $|\cdot|_{D}$ is LUR on $c_{0}([4$, Theorem 7.3, p. 69), we deduce from (17) and (19) that

therefore

$$
\lim _{n}\left|\left(\left\|y_{k}\right\|-\left\|y_{n, k}\right\|\right)_{k}\right|_{D}=0
$$

$$
\lim _{n} \max _{k}\left|\left\|y_{n, k}\right\|-\left\|y_{k}\right\|\right|=0 .
$$


For any $n \in \mathbb{N}$, let $k_{n} \in \mathbb{N}$ be such that

$$
\left\|x-x_{n}\right\|_{\infty}=\left\|y_{k_{n}}-y_{n, k_{n}}\right\| \text {. }
$$

Note that if $\lim _{n} k_{n}=+\infty$, then $\left\|x-x_{n}\right\|_{\infty} \leq 2\left\|y_{k_{n}}\right\|+\max _{k}\left|\left\|y_{n, k}\right\|-\left\|y_{k}\right\|\right|$ converges to 0 . So passing to a subsequence we may assume that $\left(k_{n}\right)_{n}$ is constant and equal to some $k_{0} \in \mathbb{N}$. If $y_{k_{0}}=0$, then by $(20), \lim _{n} y_{n, k_{0}}=0$ and $\lim _{n}\left\|x-x_{n}\right\|_{\infty}=\lim _{n}\left\|y_{k_{0}}-y_{n, k_{0}}\right\|=0$. Therefore we may assume that $y_{k_{0}} \neq 0$.

Let $m \in \mathbb{N}$ be such that $m \geq\left|\left\{i \in \mathbb{N}:\left\|y_{i}\right\| \geq \frac{1}{2}\left\|y_{k_{0}}\right\|\right\}\right|$. Let $\beta=\frac{1}{2} \frac{\left\|y_{k_{0}}\right\|}{2^{m}}$. We prove that for $n$ large enough,

$$
\left\|y_{k_{0}}+y_{n, k_{0}}\right\| \geq \beta
$$

Indeed if $(22)$ is contradicted, then it is easy to see by the expression of $|\cdot|_{D}$ that we may assume that for all $n$,

$$
\left\|x+x_{n}\right\|^{2} \leq \sum_{i=1}^{+\infty} \frac{\left\|y_{k_{i}^{n}}+y_{n, k_{i}^{n}}\right\|^{2}}{4^{i}}+\beta^{2},
$$

for some sequence $\left(k_{i}^{n}\right)_{i \geq 1}$ of distinct integers different from $k_{0}$. Let $\epsilon$ be positive. By (20) we deduce, for $n$ large enough,

$$
\left\|x+x_{n}\right\|^{2} \leq(1+\epsilon) 4 \sum_{i=1}^{+\infty} \frac{\left\|y_{k_{i}^{n}}\right\|^{2}}{4^{i}}+\beta^{2} .
$$

So

$$
\left\|x+x_{n}\right\|^{2} \leq(1+\epsilon) 4 \sum_{i=1}^{+\infty} \frac{\left\|y_{j_{i}}\right\|^{2}}{4^{i}}+\beta^{2},
$$

where $\left(\left\|y_{j_{i}}\right\|\right)_{i \geq 1}$ is a nonincreasing enumeration of $\left\{\left\|y_{k}\right\|, k \neq k_{0}\right\}$. Passing to the limit in $n$ and $\epsilon$, and using (18), we deduce

$$
4\|x\|^{2} \leq 4 \sum_{i=1}^{+\infty} \frac{\left\|y_{j_{i}}\right\|^{2}}{4^{i}}+\beta^{2} \leq 4 \sum_{i=1}^{m} \frac{\left\|y_{j_{i}}\right\|^{2}}{4^{i}}+\left\|y_{k_{0}}\right\|^{2} \sum_{i=m+1}^{+\infty} \frac{1}{4^{i}}+\beta^{2} ;
$$

consequently

$$
4\|x\|^{2}+\frac{\left\|y_{k_{0}}\right\|^{2}}{4^{m}} \leq 4\left(\sum_{i=1}^{m} \frac{\left\|y_{j_{i}}\right\|^{2}}{4^{i}}+\frac{\left\|y_{k_{0}}\right\|^{2}}{4^{m+1}}\right)+\frac{\left\|y_{k_{0}}\right\|^{2}}{3.4^{m}}+\beta^{2} \leq 4\|x\|^{2}+\frac{\left\|y_{k_{0}}\right\|^{2}}{3.4^{m}}+\beta^{2} .
$$

We deduce that $\frac{2}{3.4^{m}}\left\|y_{k_{0}}\right\|^{2} \leq \beta^{2}$, a contradiction. Therefore (22) is proved. Now

$2\|x\|^{2}+2\left\|x_{n}\right\|^{2}-\left\|x+x_{n}\right\|^{2}=2 \sum_{i=1}^{+\infty} \frac{\left\|y_{l_{i}}\right\|^{2}}{4^{i}}+2 \sum_{i=1}^{+\infty} \frac{\left\|y_{n, l_{i}^{n}}\right\|^{2}}{4^{i}}-\sum_{i=1}^{+\infty} \frac{\left\|y_{n, m_{i}^{n}}+y_{m_{i}^{n}}\right\|^{2}}{4^{i}}$,

where $\left(\left\|y_{l_{i}}\right\|\right)_{i},\left(\left\|y_{n, l_{i}^{n}}\right\|\right)_{i}$, and $\left(\left\|y_{n, m_{i}^{n}}+y_{m_{i}^{n}}\right\|\right)_{i}$ are nonincreasing enumerations of $\left(\left\|y_{k}\right\|\right)_{k},\left(\left\|y_{n, k}\right\|\right)_{k}$, and $\left(\left\|y_{k}+y_{n, k}\right\|\right)_{k}$, respectively. Hence

$2\|x\|^{2}+2\left\|x_{n}\right\|^{2}-\left\|x+x_{n}\right\|^{2} \geq 2 \sum_{i=1}^{+\infty} \frac{\left\|y_{m_{i}^{n}}\right\|^{2}}{4^{i}}+2 \sum_{i=1}^{+\infty} \frac{\left\|y_{n, m_{i}^{n}}\right\|^{2}}{4^{i}}-\sum_{i=1}^{+\infty} \frac{\left\|y_{n, m_{i}^{n}}+y_{m_{i}^{n}}\right\|^{2}}{4^{i}}$.

Since by (17) and (18),

$$
\lim _{n}\left(2\|x\|^{2}+2\left\|x_{n}\right\|^{2}-\left\|x+x_{n}\right\|^{2}\right)=0
$$


we deduce by 4 , Fact 2.3 , p. 45 that

$$
\forall i \in \mathbb{N}, \lim _{n}\left(2\left\|y_{m_{i}^{n}}\right\|^{2}+2\left\|y_{n, m_{i}^{m}}\right\|^{2}-\left\|y_{n, m_{i}^{n}}+y_{m_{i}^{n}}\right\|^{2}\right)=0 .
$$

Let $K \in \mathbb{N}$ be such that for $k>K,\left\|y_{k}\right\| \leq \frac{\beta}{4}$. By (20), we have for $n$ large enough and $k>K$,

$$
\left\|y_{k}+y_{n, k}\right\| \leq 2\left\|y_{k}\right\|+\frac{\beta}{4} \leq \frac{\beta}{2} .
$$

By (22) we deduce that for $n$ large enough, $k_{0} \in\left\{m_{1}^{n}, \ldots, m_{K}^{n}\right\}$. There exists $i$ such that $k_{0}=m_{i}^{n}$ for infinitely many $n$ 's. Therefore from (23) we deduce, passing to a subsequence,

$$
\lim _{n} 2\left\|y_{k_{0}}\right\|^{2}+2\left\|y_{n, k_{0}}\right\|^{2}-\left\|y_{k_{0}}+y_{n, k_{0}}\right\|^{2}=0 .
$$

Since the norm $\|$.$\| on Y$ is LUR, this implies by [4, Proposition 1.2, p. 42 that $\lim _{n} y_{n, k_{0}}=y_{k_{0}}$. Finally

$$
\lim _{n}\left\|x-x_{n}\right\|_{\infty}=\lim _{n}\left\|y_{k_{0}}-y_{n, k_{0}}\right\|=0 .
$$

\section{ACKNOWLEDGEMENTS}

We thank G. Godefroy and G. Lancien for their help concerning LUR-renorming theorems for Banach spaces, and G. Godefroy and V. Zizler for information about the articles [11] and [19. We are greatly indebted to the referee for his effort in reading a first version of this paper and his numerous thoughtful suggestions and comments.

\section{REFERENCES}

[1] R. Anisca, Subspaces of $L_{p}$ with more than one complex structure, Proc. Amer. Math. Soc. 131 (2003), no. 9, 2819-2829. MR.1974339 (2004d:46014)

[2] S.F. Bellenot, Banach spaces with trivial isometries, Israel Journal of Math. 56 (1986), no. 1, 89-96. MR879916 (88b:46027)

[3] J. Bourgain, Real isomorphic complex Banach spaces need not be complex isomorphic, Proc. Amer. Math. Soc. 96 (1986), no. 2, 221-226. MR818448 (87b:46012)

[4] R. Deville, G. Godefroy and V. Zizler, Smoothness and renormings in Banach spaces, Pitman Monographs and Surveys in Pure and Applied Mathematics, Longman Scientific and Technical Ed., (1993). MR1211634 (94d:46012)

[5] J. Dieudonné, Complex structures on real Banach spaces, Proc. Amer. Math. Soc. 3 (1952), no. 1, 162-164. MR.0047252 (13:849b)

[6] V. Ferenczi, Uniqueness of complex structure and real hereditarily indecomposable Banach spaces, Advances in Math. 213 (2007), no. 1, 462-488. MR2331251(2009d:46025)

[7] V. Ferenczi and E. M. Galego, Even infinite dimensional Banach spaces, J. Funct. Anal. 253, (2007), no. 2, 534-549. MR 2370088

[8] M. González and J. M. Herrera. Decompositions for real Banach spaces with small spaces of operators, Studia Math. 183 (2007), no. 1, 1-14. MR2360254(2009d:46027)

[9] Y. Gordon and R. Loewy, Uniqueness of $(\Delta)$ bases and isometries of Banach spaces, Math. Ann. 241 (1979), no. 2, 159-180. MR.534809 (80h:46016)

[10] W.T. Gowers and B. Maurey, The unconditional basic sequence problem, J. Amer. Math. Soc. 6 (1993), no. 4, 851-874. MR1201238 (94k:46021)

[11] K. Jarosz, Any Banach space has an equivalent norm with trivial isometries, Israel Journal of Math. 64 (1988), no. 1, 49-55. MR 981748 (90a:46029)

[12] N. J. Kalton, An elementary example of a Banach space not isomorphic to its complex conjugate, Canad. Math. Bull. 38 (1995), no. 2, 218-222. MR1335101(96e:46018) 
[13] A. S. Kechris, Classical Descriptive Set Theory, Graduate Texts in Mathematics, 156, Springer-Verlag, New York, 1995. MR.1321597 (96e:03057)

[14] G. Lancien, Dentability indices and locally uniformly convex renormings, Rocky Mountain J. Math. 23 (1993), no. 2, 635-647. MR.1226193 (94h:46026)

[15] J. Lindenstrauss and L. Tzafriri, Classical Banach spaces, Springer-Verlag, New York, Heidelberg, Berlin (1979). MR0415253 (54:3344)

[16] A.N. Pličko, Construction of bounded fundamental and total biorthogonal systems from unbounded systems (Russian), Dokl. Akad. Nauk Ukrain. SSR Ser. A 93 (1980), no. 5, 19-23. MR579359 (81h:46012)

[17] F. Rabiger and W.J. Ricker. $C_{0}$-groups and $C_{0}$-semigroups of linear operators on hereditarily indecomposable Banach spaces, Arch. Math. 66 (1996), no. 1, 60-70. MR.1363778(96i:47070)

[18] H.P. Rosenthal, The Banach spaces $C(K)$, Handbook of the Geometry of Banach spaces, Vol. 2, Edited by W.B. Johnson and J. Lindenstrauss, North-Holland, 2003, 1547-1602. MR.1999603 (2004g:46028)

[19] J. Stern, Le groupe des isométries d'un espace de Banach (French), Studia Math. 64 (1979), no. 2, 139-149. MR.537117 (80f:46022)

Departamento de Matemática, Instituto de Matemática e Estatística, Universidade de São Paulo, Rua do Matão, 1010 - Cidade Universitária, 05508-090 São Paulo, SP, BRAZIL

E-mail address: ferenczi@ime.usp.br

Departamento de Matemática, Instituto de Matemática e Estatística, Universidade de São Paulo, rua do Matão, 1010 - Cidade Universitária, 05508-090 São Paulo, SP, BRAZIL

E-mail address: eloi@ime.usp.br 